LPENSL-TH-05/2001

UNIL-IPT-01-13

hep-th/0110131

\title{
Superfield Noether Procedure
}

\author{
Marc Magro 凹 \\ Laboratoire de Physique, École normale supérieure de Lyon, \\ 46, Allée d'Italie, 69364 Lyon - Cedex 07, France \\ Ivo Sachs 2 \\ Theoretische Physik, Ludwig-Maximilians Universität, \\ Theresienstrasse 37, D-80333, München, Germany \\ Sylvain Wolf \\ Institut de Physique Théorique, BSP, Université de Lausanne \\ 1015 Lausanne, Switzerland
}

\begin{abstract}
We develop a superspace Noether procedure for supersymmetric field theories in 4-dimensions for which an off-shell formulation in ordinary superspace exists. In this way we obtain an elegant and compact derivation of the various supercurrents in these theories. We then apply this formalism to compute the central charges for a variety of effective actions. As a by-product we also obtain a simple derivation of the anomalous superconformal Ward-identity in $\mathcal{N}=2$ Yang-Mills theory. The connection with linearized supergravity is also discussed.
\end{abstract}

\footnotetext{
${ }^{1}$ Marc.Magro@ens-lyon.fr

${ }^{2}$ ivo@theorie.physik.uni-muenchen.de

${ }^{3}$ Sylvain.Wolf@ipt.unil.ch
} 


\section{Introduction}

Noether currents play an important role in any theory with a continuous global symmetry. Moreover, when there is more than one invariance, the various Noether currents themselves form a multiplet for the extended symmetry group. This property is used extensively in supersymmetric theories. Indeed, soon after the first 4-dimensional supersymmetric field theory was proposed by Wess and Zumino [1], the corresponding multiplet of Noether currents, containing the energy momentum tensor, the supersymmetry current and the R-current was constructed [2]. This multiplet structure plays an important role in exploring non-perturbative properties of the quantum theory. So, for example, the rigid multiplet structure of the Noether currents made it possible to obtain the $\beta$-function to all orders for minimally supersymmetric Yang-Mills theory [3, 4, 5]. Furthermore, the multiplet structure of these currents was crucial in explaining higher loop finiteness of theories with extended supersymmetry [6, 0, 8]. Similarly, the supercurrent of $\mathcal{N}=2$ Yang-Mills theory played an important role in deriving the Seiberg-Witten low energy effective action for that theory [9, 10, 11]. On another front, the Noether currents of supersymmetric matter theories can be used to construct linear off-shell supergravities [12].

As with any symmetry, the multiplet structure is best discussed by using a manifestly covariant formalism. For supersymmetric theories, the manifestly covariant formulation is in terms of superfields [13]. However, as superfields are not adapted to the canonical formalism, the approach usually followed consists of first working in components to determine the different conserved currents and then constructing the corresponding supercurrent. This can be circumvented by using a variational approach to obtain the Noether currents [14, 15, 16, 17]. However, one typically encounters constrained superfields when formulating realistic supersymmetric theories. Solving these constraints is possible for all $\mathcal{N}=1$ theories but has been worked out in ordinary superspace only for a limited subset of theories with extended supersymmetry. Thus, the variational approach is complicated by having to deal with constraint preserving variations. Alternatively, if a superspace description of the coupling of the theory in question to supergravity is available, the supercurrent can be obtained by variation with respect to the supergravity fields (see e.g. [18] for a review and references).

In spite of these drawbacks, a manifestly covariant derivation of the multiplet of Noether currents is certainly desirable. The purpose of this paper is to develop a general formalism (Superfield Noether Procedure) to determine the supercurrent associated with the super-Poincaré/superconformal invariance of a generic theory that can be formulated in terms of $\mathcal{N}$-extended, unconstrained superfields. For $\mathcal{N}=1$, a procedure to extract a supercurrent was proposed in 114, 19. Here we elaborate on a superfield Noether procedure first proposed in [16, 17]. The starting point is an abstract supersymmetric theory whose action can be expressed in terms of $\mathcal{N}$-extended superfields. We then obtain the supercurrent by considering the variation of the action under a local transformation on the superfield level. In this way we obtain directly the supercurrent for an arbitrary action of the type described above. The various component Noether currents are then obtained by an appropriate projection of the supercurrent. While this approach may not appear to be very economic to recover the known multiplets of Noether currents in the simplest models, it is rather powerful in generalizing these results to more complicated Lagrangians, such as low energy effective 
Lagrangians in supersymmetric quantum field theory and string theory. In particular, we obtain the supercurrents for a variety of $\mathcal{N}=1$ and $\mathcal{N}=2$ multiplets with arbitrary local action. Furthermore, our procedure enables us to add improvement terms at the superfield level, that is, in a manifestly supersymmetric manner. This allows us to give a uniform description of all multiplets differing by improvement terms including the so-called canonical multiplet which, among its components contains the central charges of the supersymmetry algebra. As a result we obtain an elegant and economic derivation of central charges of the supersymmetry algebra in models with arbitrary Lagrangians, not just holomorphic ones. As another simple application, we will give a simple derivation of the anomalous superconformal Ward-Identity in $\mathcal{N}=2$ Yang-Mills theory [20]. Finally, the superfield Noether procedure provides a tool for a simple construction of linearized supergravities directly at the superfield level. In particular, we recover various known, linearized $\mathcal{N}=1$ supergravities [6], [21]- 25] as well as some $\mathcal{N}=2$ supergravities [26]-30] in a simple and uniform manner.

The rest of this paper is organized as follows. In section 2, we formulate the super-Noether procedure for an abstract supersymmetric action formulated in terms of unconstrained superfields. In order to be self contained we begin with a review of the superspace diffeomorphism transformations and of the superconformal and superPoincaré subgroups. In section 3, we illustrate the use of this formalism by applying it to concrete models with $\mathcal{N}=1$ supersymmetry. In particular we obtain their supercurrents and compute the corresponding central charges. In section 4 , we then refine the general formalism to deal with the constrained superfields in $\mathcal{N}=2$ and then apply it to the $\mathcal{N}=2$ vector and tensor multiplets. As a result we obtain a simple derivation of the anomalous superconformal Ward Identity for the vector multiplet and compute the effective central charge including fermions in the theory as well as the contributions from the non-holomorphic part of the effective action for that model. For the tensor multiplet, we derive the supercurrent and discuss the central charge. In section 5, we discuss the construction of $\mathcal{N}=1$ and $\mathcal{N}=2$ linearized superfield supergravities using the superfield Noether procedure. In section 6 , we present the algebraic relations between the component Noether currents. Finally, we present the conclusions in the last section.

\section{Superfield Noether Procedure}

The purpose of this section is to develop the general formalism to obtain the supercurrent for an arbitrary super-Poincaré/superconformal invariant theory formulated in terms of unconstrained, $\mathcal{N}$-extended, superfields. To extract the Noether currents associated with the global super-Poincaré/superconformal symmetries, we need to consider the corresponding local transformations. Therefore, in the next subsection we first discuss some aspects of the larger group of superdiffeomorphisms.

\subsection{Superdiffeomorphisms}

The field theories considered in this paper are formulated on $\mathcal{N}$-extended superspace with coordinates $z=\left(x^{\mu}, \theta_{\alpha \mathbf{i}}, \bar{\theta}_{\dot{\alpha}}^{\mathbf{i}}\right) \in \mathbb{R}^{4 \mid 4 \mathcal{N}}$, i being the $S U(\mathcal{N})$ index, or, on the 
complex chiral- and anti chiral superspaces parametrized by $z_{+}=\left(x_{+}^{\mu}, \theta_{\alpha \mathbf{i}}\right)$ and $z_{-}=\left(x_{-}^{\mu}, \bar{\theta}_{\dot{\alpha}}^{\mathbf{i}}\right)$. Concretely we consider those superdiffeomorphisms which preserve chirality. Such transformations can be described in terms of a superfield $h^{\alpha \dot{\alpha}}$, subject to the constraints

$$
\bar{D}_{\mathbf{i}}^{(\dot{\beta}} h^{\alpha \dot{\alpha})}=0, \quad D^{(\beta \mathbf{i}} \bar{h}^{\alpha) \dot{\alpha}}=0
$$

The corresponding transformations of the chiral coordinates are given by

$$
\begin{array}{ll}
\delta x_{+}^{\mu}=h^{\mu}(z)+2 i \lambda_{\mathbf{i}}\left(z_{+}\right) \sigma^{\mu} \bar{\theta}^{\mathbf{i}}, & \delta \theta_{\mathbf{i}}^{\alpha}=\lambda_{\mathbf{i}}^{\alpha}\left(z_{+}\right), \\
\delta x_{-}^{\mu}=\bar{h}^{\mu}(z)-2 i \theta_{\mathbf{i}} \sigma^{\mu} \bar{\lambda}^{\mathbf{i}}\left(z_{-}\right), & \delta \bar{\theta}^{\dot{\alpha} \mathbf{i}}=\bar{\lambda}^{\dot{\alpha} \mathbf{i}}\left(z_{-}\right),
\end{array}
$$

with

$$
\lambda_{\mathbf{i}}^{\alpha}\left(z_{+}\right)=-\frac{i}{8} \bar{D}_{\dot{\alpha} \mathbf{i}} h^{\alpha \dot{\alpha}}, \quad \bar{\lambda}^{\dot{\alpha} \mathbf{i}}\left(z_{-}\right)=\frac{i}{8} D_{\alpha}^{\mathbf{i}} \bar{h}^{\alpha \dot{\alpha}} .
$$

The representation of (2.2) in terms of the corresponding differential operators acting on chiral and antichiral superfields are then

$$
\mathcal{L}_{+}=h^{\mu} \partial_{\mu}+\lambda_{\mathbf{i}}^{\alpha} D_{\alpha}^{\mathbf{i}}, \quad \mathcal{L}_{-}=\bar{h}^{\mu} \partial_{\mu}+\bar{\lambda}_{\dot{\alpha}}^{\mathbf{i}} \bar{D}_{\mathbf{i}}^{\dot{\alpha}}
$$

These commute with the chirality constraint, $\left[\bar{D}_{\dot{\alpha} \mathbf{i}}, \mathcal{L}_{+}\right]=0$ and $\left[D_{\alpha}^{\mathbf{i}}, \mathcal{L}_{-}\right]=0$, as a consequence of (2.1).

An important subgroup of the superdiffeomorphisms containing the super-Poincaré transformations is the superconformal group [32, 33] obtained by imposing the constraint

$$
h^{\alpha \dot{\alpha}}=\bar{h}^{\alpha \dot{\alpha}} .
$$

From (2.1) and (2.5), we can easily extract the Killing equation $\partial_{\mu} h_{\nu}+\partial_{\nu} h_{\mu}=\frac{1}{2} \eta_{\mu \nu} \partial^{\rho} h_{\rho}$, the general solution of which is given in appendix A.1. Finally, super-Poincaré transformations are those for which the chiral and traceless, hermitian superfields,

$$
\sigma=\frac{1}{6}\left(D^{\alpha \mathbf{i}} \lambda_{\alpha \mathbf{i}}-\frac{1}{2} \partial_{\alpha \dot{\alpha}} h^{\alpha \dot{\alpha}}\right)
$$

and

$$
\Lambda_{\mathbf{j}}^{\mathbf{i}}=-\frac{i}{4}\left(D_{\alpha}^{\mathbf{i}} \lambda_{\mathbf{j}}^{\alpha}+\bar{D}_{\dot{\alpha} \mathbf{j}} \bar{\lambda}^{\dot{\alpha} \mathbf{i}}-\frac{1}{\mathcal{N}} \delta_{\mathbf{j}}^{\mathbf{i}}\left(D_{\alpha}^{\mathbf{k}} \lambda_{\mathbf{k}}^{\alpha}+\bar{D}_{\dot{\alpha} \mathbf{k}} \bar{\lambda}^{\dot{\alpha} \mathbf{k}}\right)\right)
$$

both vanish.

\section{$2.2 \quad$ Noether Procedure}

Let us denote by $O^{A \mathbf{J}}$ a generic, unconstrained superfield, where $A$ is a collective vector and spinor index and $\mathbf{J}$ transforms in a representation of the $S U(\mathcal{N})$ internal group. On $O^{A \mathbf{J}}$, the superconformal group acts as

$$
\begin{aligned}
\delta O^{A \mathbf{J}}(z)= & -\mathcal{L} O^{A \mathbf{J}}+\left(\Omega_{\alpha}{ }^{\beta} \mathcal{S}_{\beta}{ }^{\alpha}+\bar{\Omega}_{\dot{\alpha}}{ }^{\dot{\beta}} \overline{\mathcal{S}}_{\dot{\beta}}^{\dot{\alpha}}\right)_{B}{ }^{A} O^{B \mathbf{J}} \\
& +i \Lambda_{\mathbf{j}}^{\mathbf{i}}\left(\mathcal{R}^{\mathbf{j}}{ }_{\mathbf{i}}\right)^{\mathbf{J}}{ }_{\mathbf{K}} O^{A \mathbf{K}}-2(q \sigma+\bar{q} \bar{\sigma}) O^{A \mathbf{J}},
\end{aligned}
$$

\footnotetext{
${ }^{4}$ We use essentially Wess and Bagger conventions [31, see appendix D.

${ }^{5}$ For detailed discussions of the multiplet of superconformal transformations we refer to [32][36, [16]-[18, 20]. We will give generalizations where necessary.
} 
where

$$
\mathcal{L}=\frac{1}{2}\left(h^{\mu}+\bar{h}^{\mu}\right) \partial_{\mu}+\lambda_{\mathbf{i}}^{\alpha} D_{\alpha}^{\mathbf{i}}+\bar{\lambda}_{\dot{\alpha}}^{\mathbf{i}} \bar{D}_{\mathbf{i}}^{\dot{\alpha}}
$$

The symbols $\mathcal{S}_{\beta}{ }^{\alpha}$ and $\mathcal{R}_{\mathbf{i}}^{\mathbf{j}}$ stand for the Lorentz and $S U(\mathcal{N})$ generators of the representation to which $O^{A \mathbf{J}}$ belongs and $\Omega_{\alpha}{ }^{\beta}$ is defined by eq.(A.4) in appendix A.1. The

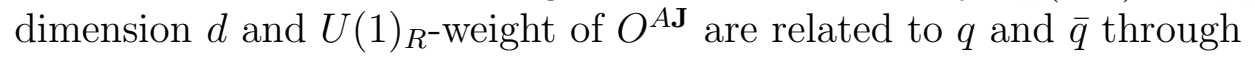

$$
d=\frac{4-\mathcal{N}}{3}(q+\bar{q}), \quad R=-\frac{2 \mathcal{N}}{3}(q-\bar{q}) .
$$

For a global super-Poincaré transformation, we have $\delta S\left[O^{A \mathbf{J}}\right]=0$, where $S\left[O^{A \mathbf{J}}\right]$ is the action. As in ordinary Noether procedure, we then consider a local transformation, by letting the parameters have an arbitrary $x$-dependence. We implement this by removing the reality condition (2.5) but still maintaining (2.1), i.e. the chirality preserving constraint. Of course, there is always the ambiguity of adding terms to $\delta O^{A \mathbf{J}}$ which are proportional to derivatives of the parameters of super-Poincaré transformations. By construction, the terms in $\delta S\left[O^{A \mathbf{J}}\right]$ induced by them are of the form "derivatives of the parameters" times "equations of motions" and thus induce in the currents terms which vanish on-shell. We will make use of this freedom when dealing with constrained superfields. Using (2.8), the variation of the action can then always be written as

$$
\begin{aligned}
\delta S\left[O^{A \mathbf{J}}\right]= & \frac{i}{16} \int d^{4+4 \mathcal{N}} z\left\{\left(h^{\alpha \dot{\alpha}}-\bar{h}^{\alpha \dot{\alpha}}\right) T_{\alpha \dot{\alpha}}+i\left(h^{\alpha \dot{\alpha}}+\bar{h}^{\alpha \dot{\alpha}}\right) K_{\alpha \dot{\alpha}}\right\} \\
& -\frac{1}{2} \int d^{4+2 \mathcal{N}} z_{+} \sigma J-\frac{1}{2} \int d^{4+2 \mathcal{N}} z_{-} \bar{\sigma} \bar{J}
\end{aligned}
$$

with $T_{\alpha \dot{\alpha}}$ and $K_{\alpha \dot{\alpha}}$ real and $J$ chiral. The vanishing of $\delta S$ for global super-Poincaré transformations furthermore implies of

$$
K_{\alpha \dot{\alpha}}=-\frac{i}{4}\left[D_{\alpha}^{\mathbf{i}}, \bar{D}_{\dot{\alpha} \mathbf{i}}\right](X-\bar{X})-\frac{\mathcal{N}}{2} \partial_{\alpha \dot{\alpha}}(X+\bar{X})
$$

for some $X$. However, the superfields $T_{\alpha \dot{\alpha}}, K_{\alpha \dot{\alpha}}$ and $J$ are not unique. Indeed, as a consequence of (2.1) we have the two identities

$$
\begin{aligned}
& -\frac{1}{2}\left[D_{\alpha}^{\mathbf{i}}, \bar{D}_{\dot{\alpha} \mathbf{i}}\right](h+\bar{h})^{\alpha \dot{\alpha}}-48 i(\sigma-\bar{\sigma})-i(4-\mathcal{N}) \partial_{\alpha \dot{\alpha}}(h-\bar{h})^{\alpha \dot{\alpha}}=0, \\
& -\frac{1}{2}\left[D_{\alpha}^{\mathbf{i}}, \bar{D}_{\dot{\alpha} \mathbf{i}}\right](h-\bar{h})^{\alpha \dot{\alpha}}-48 i(\sigma+\bar{\sigma})-i(4-\mathcal{N}) \partial_{\alpha \dot{\alpha}}(h+\bar{h})^{\alpha \dot{\alpha}}=0,
\end{aligned}
$$

which, upon integration by parts lead to the equivalence relation

$$
\left(\begin{array}{c}
T_{\alpha \dot{\alpha}} \\
K_{\alpha \dot{\alpha}} \\
J
\end{array}\right) \simeq\left(\begin{array}{c}
T_{\alpha \dot{\alpha}}+i \frac{4-\mathcal{N}}{2} \partial_{\alpha \dot{\alpha}}(A-\bar{A})-\frac{\mathcal{N}}{4(4-\mathcal{N})}\left[D_{\alpha}^{\mathbf{i}}, \bar{D}_{\dot{\alpha} \mathbf{i}}\right](A+\bar{A}) \\
K_{\alpha \dot{\alpha}}+\frac{i}{4}\left[D_{\alpha}^{\mathbf{i}}, \bar{D}_{\dot{\alpha} \mathbf{i}}\right](A-\bar{A})+\frac{\mathcal{N}}{2} \partial_{\alpha \dot{\alpha}}(A+\bar{A}) \\
J-\frac{6}{4-\mathcal{N}} \bar{D}^{2 \mathcal{N}}(2 A+(\mathcal{N}-2) \bar{A})
\end{array}\right)
$$

\footnotetext{
${ }^{6}$ For $\mathcal{N}=1$, this was shown in $[17]$. For $\mathcal{N}=2$, this can be proved following the same argument as in 17]. Note however that the $\mathcal{N}=2$ case is more elaborated than in $\mathcal{N}=1$ as one has to use not only super-Poincaré invariance but also $S U(2)_{R}$ invariance. The proof of (2.12) for arbitrary $\mathcal{N}$ should follow the same pattern.
} 
where $A$ is any unconstrained superfield. This equivalence relation corresponds to the freedom of adding improvement terms to the different Noether currents in the multiplets. One way to see that is to note that the equivalence classes (2.14) are obtained through integration by parts or equivalently, by adding boundary terms. We will explain this correspondence in more detail in the following sections.

There are two distinguished multiplets of Noether currents. We denote them by the minimal and the canonical multiplets respectively. Indeed, starting from a generic supermultiplet $\left(T_{\alpha \dot{\alpha}}, K_{\alpha \dot{\alpha}}, J\right)$ and taking $A=X$, we arrive at the minimal multiplet $\bar{\emptyset}$ $\left(T_{\alpha \dot{\alpha}}^{\min }, K_{\alpha \dot{\alpha}}^{\min }=0, J^{\min }\right)$. In particular, for a superconformal invariant theory, $\delta S=0$ for a superconformal transformation and hence (2.11) implies that $J^{\text {min }}=\bar{J}^{\text {min }}=0$. In this case the supercurrent $T_{\alpha \dot{\alpha}}^{\min }$ contains the traceless (i.e. improved) currents. This is the smallest possible multiplet of Noether currents. If the theory does not have superconformal invariance, the $K_{\alpha \dot{\alpha}}=0$ multiplet of currents is still minimal in the sense that it has the smallest number of components.

The other distinguished multiplet is the canonical multiplet. This multiplet contains the generators of the super-Poincaré transformations and is related to the minimal multiplet by total derivative terms. This multiplet has the further property that it contains among its components central charges.

Thus far the discussion applies to any theory formulated in terms of unconstrained superfields. However, as already mentioned in the introduction, in realistic situations we typically have to deal with constrained superfields. In general, the localization of the super-Poincaré or superconformal transformations in terms of an arbitrary parameter $h^{\alpha \dot{\alpha}}$ subject to (2.1) may not be compatible with the constraints on the superfield. In that case, one may have to impose stronger constraints on $h^{\alpha \dot{\alpha}}$. The purpose of the next sections is to illustrate the general procedure by applying it to some $\mathcal{N}=1$ models of interest and to discuss the modifications for $\mathcal{N}=2$ models with off-shell superfield formulations.

\section{$3 \mathcal{N}=1$ Theories}

In this section, we will apply the general formalism of section 2 to an arbitrary $\mathcal{N}=1$ theory formulated in terms of (possibly constrained) $\mathcal{N}=1$ superfields. Indeed, it turns out that the general structure described above applies directly without modification to any $\mathcal{N}=1$ theory with an off-shell superfield formulation.

\subsection{General Structure}

Before considering concrete models with $\mathcal{N}=1$ supersymmetry we present some model independent, general results in this subsection.

\subsubsection{Equation of Conservation}

We begin by solving the constraint (2.1) in terms of a free spinor superfield $L^{\alpha}$ as

$$
h^{\alpha \dot{\alpha}}=2 \bar{D}^{\dot{\alpha}} L^{\alpha}, \quad \bar{h}^{\alpha \dot{\alpha}}=-2 D^{\alpha} \bar{L}^{\dot{\alpha}} .
$$

\footnotetext{
${ }^{7}$ In general, this minimal multiplet may not be unique [17, but this will not affect our analysis below.
} 
Using the results in section 2 we can now write the variation of the action under an arbitrary local transformation parametrized by $L^{\alpha}$ as $\underbrace{8}$ [16, 17]

$$
\begin{aligned}
\delta S & =\int d^{8} z\left(\frac{i}{8} L^{\alpha} R_{\alpha}+\text { c.c. }\right), \quad \text { with } \\
R_{\alpha} & =\bar{D}^{\dot{\alpha}} T_{\alpha \dot{\alpha}}+i \bar{D}^{\dot{\alpha}} K_{\alpha \dot{\alpha}}-\frac{1}{6} D_{\alpha} J,
\end{aligned}
$$

where $K_{\alpha \dot{\alpha}}$ is given by (2.12). Variation with respect to $L^{\alpha}$ then leads to the supercurrent conservation equation

$$
\bar{D}^{\dot{\alpha}} T_{\alpha \dot{\alpha}}+i \bar{D}^{\dot{\alpha}} K_{\alpha \dot{\alpha}}-\frac{1}{6} D_{\alpha} J=0
$$

As explained in section 2, the multiplets appearing in (3.3) are not unique. In particular, we can always make the choice $K_{\alpha \dot{\alpha}}=0$ provided we redefine $T_{\alpha \dot{\alpha}}$ and $J$ as

$$
\begin{aligned}
T_{\alpha \dot{\alpha}} & \rightarrow \widetilde{T}_{\alpha \dot{\alpha}}=T_{\alpha \dot{\alpha}}+\frac{3 i}{2} \partial_{\alpha \dot{\alpha}}(X-\bar{X})-\frac{1}{12}\left[D_{\alpha}, \bar{D}_{\dot{\alpha}}\right](X+\bar{X}) \quad \text { and } \\
J & \rightarrow \widetilde{J}=J-2 \bar{D}^{2}(2 X-\bar{X})
\end{aligned}
$$

where we have replaced $\min$ by $\sim$ to avoid clutter. The supercurrent conservation equation then reads

$$
R_{\alpha}=\bar{D}^{\dot{\alpha}} \widetilde{T}_{\alpha \dot{\alpha}}-\frac{1}{6} D_{\alpha} \widetilde{J}=0 .
$$

In particular, if the theory has superconformal invariance, there is a minimal multiplet with $\tilde{J}=0$. In general, $\tilde{J}$ contains the trace of the energy momentum tensor and the supersymmetry current and is then referred to as the multiplet of anomalies [21, 37]. Using (2.12) we can recast the conservation equation (3.3) in a more familiar form

$$
\bar{D}^{\dot{\alpha}} T_{\alpha \dot{\alpha}}+W_{\alpha}+D_{\alpha} \tau=0
$$

where

$$
W_{\alpha}=\frac{1}{4} \bar{D}^{2} D_{\alpha}(X-2 \bar{X}) \quad, \quad \tau=-\frac{1}{6} J+\frac{1}{4} \bar{D}^{2} X
$$

This form of the conservation equation is familiar from the discussion of the old- and new minimal multiplets [21, 37, 22] which will be reviewed in section 3.1.3.

\subsubsection{R-invariance}

The variation of the action under a global $R$-transformation, corresponding to $L^{\alpha}=$ $-\eta \theta^{\alpha} \bar{\theta}^{2}$ is given by

$$
\delta S=\int d^{4} x \eta\left(2 \partial^{\alpha \dot{\alpha}} \widetilde{T}_{\alpha \dot{\alpha}}-\frac{i}{6}\left(D^{2} \widetilde{J}-\bar{D}^{2} \overline{\widetilde{J}}\right)\right),
$$

\footnotetext{
${ }^{8}$ In this paper, we will take the localization of superconformal transformations even for a theory which is only super-Poincaré invariant. This is because a free superfield, playing the analogous role of $L_{\alpha}$ but for super-Poincaré transformations, is not known.
} 
and as expected, this is not automatically a derivative as we are not necessarily dealing with a $R$-invariant theory. Indeed, the $R$-invariance condition can be written as

$$
D^{2} \widetilde{J}-\bar{D}^{2} \overline{\widetilde{J}}=24 i \partial^{\mu} Z_{\mu}
$$

for some $Z_{\mu}$. If this condition is satisfied, we can define a conserved $R$-current by

$$
j_{\mu}^{(5)}=-4\left(\widetilde{T}_{\mu}-Z_{\mu}\right) \mid
$$

The condition (3.9) is solved by

$$
\widetilde{J}=\bar{D}^{2} U \quad \text { and } \quad \overline{\widetilde{J}}=D^{2} U,
$$

for some real superfield $U$. Correspondingly, we have $Z_{\alpha \dot{\alpha}}=\frac{1}{3}\left[D_{\alpha}, \bar{D}_{\dot{\alpha}}\right] U$. Now, applying (2.14) with $A=X+2 U$ we get

$$
\left(\begin{array}{l}
T_{\alpha \dot{\alpha}} \\
W_{\alpha} \\
\tau
\end{array}\right) \rightarrow\left(\begin{array}{c}
T_{\alpha \dot{\alpha}}^{\text {new }}=\widetilde{T}_{\alpha \dot{\alpha}}-\frac{1}{3}\left[D_{\alpha}, \bar{D}_{\dot{\alpha}}\right] U \\
W_{\alpha}^{\text {new }}=\frac{1}{2} \bar{D}^{2} D_{\alpha} U \\
0
\end{array}\right)
$$

where we introduced the label new for the multiplet (3.12). This is because (3.12) is, in fact, the new minimal multiplet [22. We will come back to this below. The conservation equation (3.6) then reads

$$
\bar{D}^{\dot{\alpha}} T_{\alpha \dot{\alpha}}^{\text {new }}+W_{\alpha}^{\text {new }}=0,
$$

where $W_{\alpha}^{\text {new }}$ is now a curl $\left(D^{\alpha} W_{\alpha}^{\text {new }}+\bar{D}^{\dot{\alpha}} \bar{W}_{\dot{\alpha}}^{\text {new }}=0\right)$. Therefore

$$
\partial^{\alpha \dot{\alpha}} T_{\alpha \dot{\alpha}}^{n e w}=0
$$

and consequently the conserved $R$-current is given by $T_{\alpha \dot{\alpha}}^{n e w} \mid$ which agrees with (3.10).

\subsubsection{Multiplets of Noether currents}

It is clear that the size of the multiplet of currents depends on the details of the theory under consideration but also on the choice of improvement terms. If the theory is conformally invariant, then the variation (2.11) must vanish for $h^{\alpha \dot{\alpha}}=\bar{h}^{\alpha \dot{\alpha}}$ and hence it is possible to choose $K_{\alpha \dot{\alpha}}=0$ and $J=0$. In this case, the multiplet of Noether currents is contained entirely in $T_{\alpha \dot{\alpha}}$ subject to the conservation equation $D^{\dot{\alpha}} T_{\alpha \dot{\alpha}}=0$. This is the improved multiplet with $8+8$ components first constructed by Ferarra and Zumino [2] for the Wess-Zumino model. The next bigger multiplet is described by $T_{\alpha \dot{\alpha}}$ and $J$. This is the old minimal multiplet [21, 37] with $12+12$ components. Another possibility is that $\tau$ vanishes but not $W_{\alpha}$. If $W_{\alpha}$ is a curl then we obtain the new minimal multiplet [22] with again $12+12$ components. This multiplet is distinguished in that it contains a conserved $R$-current as explained in subsection 3.1.2. If $W_{\alpha}$ is not a curl then this multiplet has $16+16$ components. If we furthermore include $J$, we obtain a multiplet with $20+20$ components. Finally, for a general $X$, we obtain a multiplet with $28+28$ components. 


\subsection{Applications}

In this subsection, we illustrate the general formalism by applying it to some concrete models with $\mathcal{N}=1$ supersymmetry. As we shall see, the super-Noether procedure provides an efficient tool to obtain the supercurrent and the central charge for general local (effective) actions given in terms of arbitrary Kähler- and prepotentials.

\subsubsection{Kähler Sigma Models}

Here we compute the supercurrent for the $\mathcal{N}=1$ sigma model defined in terms of arbitrary real Kähler potential $K(\phi, \bar{\phi})$ and superpotential $\mathcal{W}(\phi)$, where $\phi$ is a chiral scalar field. Such Lagrangians arise as the local part of quantum effective actions for supersymmetric field theories and string theory. The general action is given by

$$
S=\frac{1}{16} \int d^{8} z K(\phi, \bar{\phi})-\frac{1}{4} \int d^{6} z_{+} \mathcal{W}(\phi)-\frac{1}{4} \int d^{6} z_{-} \overline{\mathcal{W}}(\bar{\phi})
$$

with the corresponding equation of motion

$$
\mathcal{E} \equiv \frac{1}{16} \bar{D}^{2} K_{\phi}-\frac{1}{4} \mathcal{W}_{\phi}=0
$$

where a subscript $\phi$ stands for differentiation by $\phi$. According to (2.8), the transformation law for $\phi$ under global super-Poincaré/superconformal transformations is $\delta \phi=$ $-\mathcal{L}_{+} \phi-2 q \sigma \phi$, where $q$ is related to the $R$-weight and dimension of $\phi$ through (2.10). Expressing this transformation in terms of $L^{\alpha}$, we have

$$
\delta \phi=\frac{i}{4} \bar{D}^{2}\left(L^{\alpha} D_{\alpha} \phi+\frac{1}{3} q D^{\alpha} L_{\alpha} \phi\right)
$$

Substitution into (3.15) then leads to the variation of the form (3.2) with

$$
R_{\alpha}=2 D_{\alpha} \phi \mathcal{E}-\frac{2}{3} q D_{\alpha}(\phi \mathcal{E})
$$

from which we get

$$
\begin{aligned}
\widetilde{T}_{\alpha \dot{\alpha}} & =\frac{1}{12} D_{\alpha} \phi \bar{D}_{\dot{\alpha}} \bar{\phi} K_{\phi \bar{\phi}}-\frac{i}{6} \partial_{\alpha \dot{\alpha}} \phi K_{\phi}+\frac{i}{6} \partial_{\alpha \dot{\alpha}} \bar{\phi} K_{\bar{\phi}} \\
\widetilde{J} & =-\frac{1}{4} \bar{D}^{2}\left(K-q \phi K_{\phi}\right)+3 \mathcal{W}-q \phi \mathcal{W}_{\phi} .
\end{aligned}
$$

Referring to the discussion in subsection 3.1.2, we infer that the existence of a conserved $R$-current requires that $\widetilde{J}, \overline{\widetilde{J}}$ can be written in the form (3.11). This, in turn implies

$$
3 \mathcal{W}-q \phi \mathcal{W}_{\phi}=0 \quad \text { and } \quad K(\phi, \bar{\phi})=H(\phi \bar{\phi})
$$

for some function $H(\phi \bar{\phi})$. Superconformal invariance requires $\widetilde{J}=0$ which implies the same condition as in $(3.20)$ on $\mathcal{W}$, but furthermore that $K-q \phi K_{\phi}=0$. The solution of these two conditions are given by $K(\phi, \bar{\phi}) \propto(\phi \bar{\phi})^{-1 / q}$ and $\mathcal{W}(\phi) \propto \phi^{-3 / q}$. Thus, there is a superconformal model for each value of $q$. In particular, for the Wess-Zumino model, where $K(\phi, \bar{\phi})=\phi \bar{\phi}$, the superconformal invariance is achieved for $q=-1$ and $\mathcal{W}(\phi)=g \phi^{3}$, for some coupling constant $g$. 


\subsubsection{Supersymmetric $Q E D$}

We now discuss the supercurrent of $\mathcal{N}=1 Q E D$ in our formalism. This will serve as a useful preparation for dealing with the constrained superfield of $\mathcal{N}=2$ Yang-Mills discussed in the next section.

The $\mathcal{N}=1$ gauge multiplet is described by a curl superfield $W_{\alpha}$ satisfying the constraints

$$
\bar{D}_{\dot{\alpha}} W_{\alpha}=0, \quad D_{\alpha} \bar{W}_{\dot{\alpha}}=0, \quad D^{\alpha} W_{\alpha}=\bar{D}_{\dot{\alpha}} \bar{W}^{\dot{\alpha}} .
$$

These constraints are solved in terms of a real prepotential $V$ by

$$
W_{\alpha}=-\frac{1}{4} \bar{D}^{2} D_{\alpha} V .
$$

$W_{\alpha}$ is invariant under the gauge transformations:

$$
\delta_{\mathcal{G}} V=i(\Lambda-\bar{\Lambda}), \quad \text { with } \quad D_{\alpha} \bar{\Lambda}=0, \quad \bar{D}_{\dot{\alpha}} \Lambda=0,
$$

and the free action is given by

$$
S_{W}=\frac{1}{4} \int d^{6} z_{+} W^{\alpha} W_{\alpha}+\text { c.c. . }
$$

To couple this $\mathcal{N}=1$ gauge multiplet to a chiral multiplet $\phi$, we first recall its gauge transformations

$$
\delta_{\mathcal{G}} \phi=-i g \Lambda \phi, \quad \delta_{\mathcal{G}} \bar{\phi}=i g \bar{\phi} \bar{\Lambda} .
$$

The corresponding invariant action is then given by

$$
S_{\phi}=\frac{1}{16} \int d^{8} z\left(\bar{\phi} \mathrm{e}^{g V} \phi\right) .
$$

For global superconformal transformations, we have (2.8)

$$
\delta V=-\mathcal{L} V-2(q \sigma+\bar{q} \bar{\sigma}) V,
$$

with $q=\bar{q}$ since $V$ is real. The gauge potential $A_{\mu}=\frac{1}{2} \sigma_{\mu}^{\alpha \dot{\alpha}}\left[D_{\alpha}, \bar{D}_{\dot{\alpha}}\right] V \mid$ is of dimension 1 and hence $V$ has to be of dimension 0 , which implies that we take $q=0$. As explained in [17] the gauge covariant localization of (3.27) reads

$$
\delta V=-\mathcal{L} V-\frac{i}{8}(h-\bar{h})^{\alpha \dot{\alpha}}\left[D_{\alpha}, \bar{D}_{\dot{\alpha}}\right] V,
$$

up to gauge transformations. In particular, it is convenient to add to (3.28) a pure gauge term of the form

$$
-\frac{i}{4} \bar{D}^{2}\left(L^{\alpha} D_{\alpha} V\right)+\frac{i}{4} D^{2}\left(\bar{L}^{\dot{\alpha}} \bar{D}_{\dot{\alpha}} V\right) .
$$

Combining the two contributions we end up with

$$
\delta V=i\left(L^{\alpha} W_{\alpha}-\bar{L}^{\dot{\alpha}} \bar{W}_{\dot{\alpha}}\right) .
$$


As we have added a gauge transformation with parameter $\Lambda=-\frac{1}{4} \bar{D}^{2}\left(L^{\alpha} D_{\alpha} V\right)$ to the transformation law for $V$, we have to add the corresponding modification to the transformation law for $\phi$. Thus (3.17) is modified into

$$
\begin{aligned}
\delta \phi & =\frac{i}{4} \bar{D}^{2}\left(L^{\alpha}\left(D_{\alpha} \phi+g D_{\alpha} V \phi\right)+\frac{1}{3} q D^{\alpha} L_{\alpha} \phi\right) \\
& =\frac{i}{4} \bar{D}^{2}\left(L^{\alpha} \nabla_{\alpha} \phi+\frac{1}{3} q D^{\alpha} L_{\alpha} \phi\right)
\end{aligned}
$$

where $\nabla_{\alpha} \phi=D_{\alpha} \phi+g D_{\alpha} V \phi$ is the gauge covariant derivative for $\phi$ [38, 16]. Alternatively, (3.31) can be written as

$$
\delta \phi=\left(\frac{1}{2} h^{\alpha \dot{\alpha}} \nabla_{\alpha \dot{\alpha}}-\lambda^{\alpha} \nabla_{\alpha}-2 q \sigma\right) \phi
$$

with the gauge covariant space-time derivative円 $\nabla_{\alpha \dot{\alpha}} \phi=\partial_{\alpha \dot{\alpha}} \phi-\frac{i}{2} g \bar{D}_{\dot{\alpha}} D_{\alpha} V \phi$.

In order to extract the supercurrent for the combined theory $S=S_{W}+S_{\phi}$ we then apply the, by now, standard procedure and obtain a variation of the form (3.2) with

$$
\begin{aligned}
& R_{\alpha}=-\frac{1}{2} W_{\alpha} \mathcal{E}_{W}+2 \nabla_{\alpha} \phi \mathcal{E}_{\bar{\phi}}-\frac{2}{3} q D_{\alpha}\left(\phi \mathcal{E}_{\bar{\phi}}\right) \\
& \text { where }\left\{\begin{array}{l}
\mathcal{E}_{W}=\frac{1}{8}\left(D^{\alpha} W_{\alpha}+\bar{D}_{\dot{\alpha}} \bar{W}^{\dot{\alpha}}\right)+\frac{1}{16} g\left(\bar{\phi} \mathrm{e}^{g V} \phi\right), \\
\mathcal{E}_{\bar{\phi}}=\frac{1}{16} \bar{D}^{2}\left(\mathrm{e}^{g V} \bar{\phi}\right)
\end{array}\right.
\end{aligned}
$$

are the equations of motion for the gauge field and the chiral matter field, respectively. For the first term in $R_{\alpha}$, which depends only on the gauge superfield, we can use the constraints (3.21) to write it as

$$
R_{\alpha}^{W}=-\frac{1}{8} W_{\alpha} \bar{D}_{\dot{\alpha}} \bar{W}^{\dot{\alpha}}=-\frac{1}{8} \bar{D}^{\dot{\alpha}}\left(W_{\alpha} \bar{W}_{\dot{\alpha}}\right)
$$

which shows that for the Maxwell supermultiplet we have $\widetilde{T}_{\alpha \dot{\alpha}}^{W}=-\frac{1}{8} W_{\alpha} \bar{W}_{\dot{\alpha}}$ and $\widetilde{J}^{W}=0$.

For the matter part of the supercurrent we proceed by analogy with the WessZumino case, but taking into account that the theory is now gauge invariant. For this, we first define $\Phi=\mathrm{e}^{\frac{g}{2} V} \phi$ so that the invariant matter action takes the same form as for the Wess-Zumino model ( 3.15 ) with $K=\Phi \bar{\Phi}$ and $\mathcal{W}=0): S_{\phi}=\frac{1}{16} \int d^{8} z \bar{\Phi} \Phi$. This suggests that the supercurrent is just the one for the Wess-Zumino model, (3.19), but with derivatives replaced by covariant ones [16]:

$$
\begin{aligned}
\widetilde{T}_{\alpha \dot{\alpha}}^{\phi} & =\frac{1}{12} \nabla_{\alpha} \Phi \bar{\nabla}_{\dot{\alpha}} \bar{\Phi}-\frac{i}{6} \bar{\Phi} \overleftrightarrow{\nabla}_{\alpha \dot{\alpha}} \Phi \\
\widetilde{J}^{\phi} & =\frac{1}{4}(q-1) \bar{\nabla}^{2}(\bar{\Phi} \Phi) .
\end{aligned}
$$

Plugging this into (3.5) then shows that this is the correct answer, i.e. we recover (3.33). Thus, the gauge invariant supercurrent for the $\mathcal{N}=1$ abelian YM theory coupled to a

\footnotetext{
${ }^{9}$ For a general gauge covariant expression $X$ transforming as $\delta_{\mathcal{G}} X=i g(e \Lambda-\bar{e} \bar{\Lambda}) X$, the gauge covariant derivatives are defined as $\nabla_{\alpha} X=D_{\alpha} X-e g D_{\alpha} V X$ and $\bar{\nabla}_{\dot{\alpha}} X=\bar{D}_{\dot{\alpha}} X-\bar{e} g \bar{D}_{\dot{\alpha}} V X$. Note that we still have $\bar{\nabla}_{\dot{\alpha}} \phi=0$, i.e. $\phi$ is gauge covariantly chiral. The covariant derivative $\nabla_{\alpha \dot{\alpha}}$ acts on $X$ as $\nabla_{\alpha \dot{\alpha}} X=\partial_{\alpha \dot{\alpha}} X-\frac{i}{2} g\left(e \bar{D}_{\dot{\alpha}} D_{\alpha} V+\bar{e} D_{\alpha} \bar{D}_{\dot{\alpha}} V\right) X$.
} 
scalar multiplet is

$$
\begin{aligned}
\widetilde{T}_{\alpha \dot{\alpha}} & =-\frac{1}{8} W_{\alpha} \bar{W}_{\dot{\alpha}}+\frac{1}{12} \nabla_{\alpha} \Phi \bar{\nabla}_{\dot{\alpha}} \bar{\Phi}-\frac{i}{6} \bar{\Phi} \stackrel{\leftrightarrow}{\nabla}_{\alpha \dot{\alpha}} \Phi \\
\widetilde{J} & =\frac{1}{4}(q-1) \bar{\nabla}^{2}(\bar{\Phi} \Phi) .
\end{aligned}
$$

\subsubsection{Tensor Multiplet}

The last application we consider is the tensor multiplet 39 described by a superfield $G$ constrained by:

$$
D^{2} G=0, \quad \bar{D}^{2} G=0, \quad G=\bar{G} .
$$

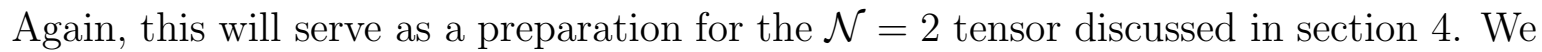
consider a general action $S=\int d^{8} z \mathcal{F}(G)$ depending on an arbitrary function $\mathcal{F}$. There are two distinguished cases. The first one is the free field action $\mathcal{F}(G)=G^{2}$ which is $R$-invariant for a suitable choice of the $R$-weight of $G$, but is not conformal invariant for any choice of the conformal weight. The second model has $\mathcal{F}(G)=G \log G$ 40. This model is superconformal invariant for a suitable choice of the conformal weight of G.

The constraints (3.37) are solved by

$$
G=D^{\alpha} \phi_{\alpha}+\bar{D}_{\dot{\alpha}} \bar{\phi}^{\dot{\alpha}}
$$

where $\phi_{\alpha}$ is an unconstrained chiral spinor superfield. Moreover, as expected, there is a gauge freedom of the form $\delta_{\mathcal{G}} \phi_{\alpha}=\bar{D}^{2} D_{\alpha} K$, with $K=\bar{K}$. In analogy with the vector multiplet in the last subsection, we find for the gauge covariant localization of the global symmetry transformation of $\phi_{\alpha}$

$$
\begin{aligned}
\delta \phi_{\alpha} & =-\mathcal{L} \phi_{\alpha}-3 \sigma \phi_{\alpha}+\Omega_{\alpha}{ }^{\beta} \phi_{\beta}-\frac{i}{8} \bar{D}^{2}\left(\left(h_{\alpha \dot{\alpha}}-\bar{h}_{\alpha \dot{\alpha}}\right) \bar{\phi}^{\dot{\alpha}}\right)+\frac{i}{4} \bar{D}^{2} D_{\alpha}\left(L^{\beta} \phi_{\beta}+\bar{L}_{\dot{\beta}} \bar{\phi}^{\dot{\beta}}\right) \\
& =-\frac{i}{4} \bar{D}^{2}\left(L_{\alpha} G\right)
\end{aligned}
$$

corresponding to $q=\frac{3}{2}$, which is the only choice which leads to a gauge invariant

transformation for $G$. The equation of motion for $G$ reads $\mathcal{E}_{\alpha}=-\bar{D}^{2} D_{\alpha} \mathcal{F}^{\prime}$, where the prime denotes, as usual, the derivative of $\mathcal{F}$ w.r.t. $G$. The computation of the supercurrent conservation equation is now straightforward and we end up with

$$
R_{\alpha}=-2 G \mathcal{E}_{\alpha}=2 G \bar{D}^{2} D_{\alpha} \mathcal{F}^{\prime}=-2 \bar{D}^{\dot{\alpha}}\left(G \bar{D}_{\dot{\alpha}} D_{\alpha} \mathcal{F}^{\prime}-\bar{D}_{\dot{\alpha}} G D_{\alpha} \mathcal{F}^{\prime}\right)
$$

It is then just a matter of separating the real part from the imaginary one to get

$$
\begin{aligned}
T_{\alpha \dot{\alpha}} & =-2 D_{\alpha} G \bar{D}_{\dot{\alpha}} G \mathcal{F}^{\prime \prime}+G\left[D_{\alpha}, \bar{D}_{\dot{\alpha}}\right] \mathcal{F}^{\prime} \\
K_{\alpha \dot{\alpha}} & =2 G \partial_{\alpha \dot{\alpha}} \mathcal{F}^{\prime}=-2 \partial_{\alpha \dot{\alpha}}\left(\mathcal{F}-G \mathcal{F}^{\prime}\right), \\
J & =0 .
\end{aligned}
$$

From these objects, we finally obtain the minimal multiplet:

$$
\begin{aligned}
\widetilde{T}_{\alpha \dot{\alpha}} & =-\frac{4}{3} D_{\alpha} G \bar{D}_{\dot{\alpha}} G\left(\mathcal{F}^{\prime \prime}-2 G \mathcal{F}^{\prime \prime \prime}\right)+\frac{4}{3} G \mathcal{F}^{\prime \prime}\left[D_{\alpha}, \bar{D}_{\dot{\alpha}}\right] G, \\
\widetilde{J} & =4 \bar{D}^{2}\left(G \mathcal{F}^{\prime}-\mathcal{F}\right) .
\end{aligned}
$$


We immediately see that $\widetilde{J}$ fulfills the $R$-invariance condition $(3.9)$ with $U=4\left(G \mathcal{F}^{\prime}-\mathcal{F}\right)$ and, correspondingly, $Z_{\mu}=\frac{4}{3} \sigma_{\mu}^{\alpha \dot{\alpha}}\left[D_{\alpha}, \bar{D}_{\dot{\alpha}}\right]\left(\mathcal{F}-G \mathcal{F}^{\prime}\right)$. Thus, the model is $R$-invariant for any function $\square \mathcal{F}(G)$.

For superconformal invariance, we have to impose $\widetilde{J}=0$, which leads to the differential equation $\bar{D}^{2}\left(\mathcal{F}-G \mathcal{F}^{\prime}\right)=0$ whose solutions are $\mathcal{F}(G)=G$ which is not physically interesting and $\mathcal{F}(G)=G \log G$. Thus, the action $S=\int d^{8} z(G \log G)$ is indeed superconformal invariant.

\subsection{Central Charge}

In $\mathcal{N}=1$, the central charges are often ignored as they are not compatible with translation invariance. However, they play an important role, for example, for domain walls [41, 42]. In this subsection we obtain the central charge as a certain projection of the supercurrent. This approach is much simpler than computing the central charges from the Poisson brackets of the component Noether currents. Indeed, the only place where components are needed is to identify the supersymmetry current among the components of the supercurrent. Its variation, which contains the center, is then easily obtained in superspace.

To extract the supersymmetry current we take $L^{\alpha}=-i \bar{\theta}^{2} \varepsilon^{\alpha}\left(x_{+}\right)$and $\bar{L}^{\dot{\alpha}}=-2 i \bar{\theta}^{\dot{\alpha}} \theta^{\alpha} \varepsilon_{\alpha}\left(x_{-}\right)$. Then

$$
\delta S=\int d^{4} x \varepsilon^{\alpha}\left(-2 i\left(\sigma^{\nu} \bar{\sigma}^{\mu}\right)_{\alpha}{ }^{\beta} D_{\beta} \partial_{\mu} \widetilde{T}_{\nu}+\frac{i}{3} \sigma_{\alpha}^{\mu}{ }_{\alpha}^{\dot{\beta}} \bar{D}_{\dot{\beta}} \partial_{\mu} \overline{\widetilde{J}}\right) \mid
$$

Thus, the supersymmetry current is given by

$$
\begin{aligned}
j_{\mu \alpha} & =\left(2 i\left(\sigma^{\nu} \bar{\sigma}_{\mu}\right)_{\alpha}{ }^{\beta} D_{\beta} \widetilde{T}_{\nu}+\frac{i}{3} \sigma_{\mu \alpha \dot{\alpha}} \bar{D}^{\dot{\alpha}} \overline{\widetilde{J}}+\sigma_{\mu \nu \alpha}{ }^{\beta} \partial^{\nu} D_{\beta}\left(a_{1}(X+\bar{X})+a_{2}(X-\bar{X})\right)\right) \mid \\
& =\sigma_{\mu}{ }^{\nu}{ }^{\beta}{ }^{\beta} D_{\beta}\left(-8 i \widetilde{T}_{\nu}+\partial_{\nu}\left(a_{1}(X+\bar{X})+a_{2}(X-\bar{X})\right)\right) \mid
\end{aligned}
$$

where we have used the conservation equation in the second equality. The two complex constants $a_{1}$ and $a_{2}$ introduced here correspond to improvement terms for the supersymmetry current as they are automatically conserved. It is not hard to see that the improvement terms in (3.44) correspond to different choices for $T_{\alpha \dot{\alpha}}, K_{\alpha \dot{\alpha}}$ and $J$ in (2.14). Indeed, for a generic choice, the supersymmetry current is given by

$$
j_{\mu \alpha}=2 i\left(\sigma^{\nu} \bar{\sigma}_{\mu}\right)_{\alpha}^{\beta} D_{\beta} T_{\nu}-2 i\left(\sigma_{\mu}\right)_{\alpha \dot{\alpha}} \bar{D}^{\dot{\alpha}} \bar{\tau}+\frac{i}{2}\left(\sigma_{\mu}\right)_{\alpha}{ }^{\dot{\alpha}} D^{2} \bar{D}_{\dot{\alpha}}(2 \bar{X}-X) .
$$

A short computation then shows that applying (2.14) with $A+\bar{A}=a_{1}(X+\bar{X})$ and $A-\bar{A}=a_{2}(X-\bar{X})$ on (3.45), reproduces the supersymmetry current (3.44). For $a_{1}=a_{2}=0$, the supersymmetry current (3.44) is part of the minimal multiplet as defined in section 2. A precise discussion of the multiplet structure will be given in section 6. For our purpose, the only relevant part of the algebra is the following variation of the supersymmetry current

$$
\delta_{\alpha} j_{\mu \beta}=\sigma_{\mu \nu \alpha \beta} \partial^{\nu}\left(\frac{8}{3} \overline{\widetilde{J}}+\frac{1}{2} D^{2}\left(a_{1}(X+\bar{X})+a_{2}(X-\bar{X})\right)\right) \mid .
$$

\footnotetext{
10 This is expected as $G$, being real, is not charged under $R$-symmetry.
} 


\subsubsection{Center for Kähler Sigma Models}

According to (3.19) and to (3.44), we have

$$
\begin{aligned}
j_{\mu \alpha}=\left(\sigma_{\mu}{ }^{\nu}{ }_{\alpha}{ }^{\beta} D_{\beta}(\right. & \frac{1}{6} \sigma_{\nu}^{\gamma \dot{\gamma}} D_{\gamma} \phi \bar{D} \dot{\gamma} \bar{\phi} K_{\phi \bar{\phi}}-\frac{2 i}{3} \partial_{\nu} \phi K_{\phi}+\frac{2 i}{3} \partial_{\nu} \bar{\phi} K_{\bar{\phi}} \\
& \left.\left.+\partial_{\nu}\left(a_{1}(X+\bar{X})+a_{2}(X-\bar{X})\right)\right)\right) \mid
\end{aligned}
$$

To compute the central charge for this model, we first need to extract the canonical supersymmetry current. The canonical current can be characterized by the absence of space-time derivatives on fermions. That is, the second term in (3.47) must be canceled by the improvement terms, i.e. $a_{1}(X+\bar{X})+a_{2}(X-\bar{X})=\frac{2 i}{3} K$. Thus,

$$
j_{\mu \alpha}^{c a n}=\sigma_{\mu}^{\nu}{ }^{\beta}{ }^{\beta} D_{\beta}\left(\frac{1}{6} \sigma_{\nu}^{\gamma \dot{\gamma}} D_{\gamma} \phi \bar{D}_{\dot{\gamma}} \bar{\phi} K_{\phi \bar{\phi}}+\frac{4 i}{3} \partial_{\nu} \bar{\phi} K_{\bar{\phi}}\right) \mid
$$

where "can" labels the canonical Noether current. Using (3.46), we then read off the result for the central charge $\mathcal{Z}_{(\alpha \beta)}=\int d^{3} x \delta_{(\alpha} j_{\beta)}^{\text {can } 0}$ as

$$
\mathcal{Z}_{(\alpha \beta)}=\int d^{3} x \sigma_{\alpha \beta}^{0 i} \partial_{i}\left(-\frac{4 i}{3} \overline{\widetilde{J}}+\frac{i}{3} D^{2} K\right)\left|=-3 \int d^{3} x \sigma_{\alpha \beta}^{0 i} \partial_{i} \overline{\mathcal{W}}\right|
$$

This is in agreement with the result found in [41] but without the "ambiguous term" which was due to the fact that in [41] the minimal rather than the canonical multiplet was used in the computation of the central charge. For the canonical multiplet, which is the correct multiplet to use to compare with the canonical formalism, these extra terms are absent as it should be.

\subsubsection{Center for the Tensor Multiplet}

Following the same road as for the Kähler Sigma Models, we find $a_{1}(X+\bar{X})+a_{2}(X-$ $\bar{X})=-\frac{64}{3}\left(G \mathcal{F}^{\prime}-\mathcal{F}\right)$ and the canonical supersymmetry current reads $\square$

$$
j_{\mu \alpha}^{c a n}=-8 i \sigma_{\mu}^{\beta \dot{\beta}} D_{\alpha}\left(\mathcal{F}^{\prime \prime} D_{\beta} G \bar{D}_{\dot{\beta}} G\right)
$$

Finally, using (3.46) shows that the central charge for this model identically vanishes. To summarize, we have shown how the Superfield Noether Procedure works for a variety of $\mathcal{N}=1$ theories including constrained superfields. The simplicity is due to the fact that, except for the identification of the supersymmetry current, all manipulations have been done at the superspace level. Of course, to determine the remaining Noether currents and their algebra one needs to go to component fields. We postpone this discussion to the section 6 where the currents and their corresponding multiplet structure are discussed in detail.

\footnotetext{
${ }^{11}$ After adding an equation of motion.
} 


\section{$4 \mathcal{N}=2$ Theories}

In this section, we will explain how the general procedure developed in section 2 has to be refined in order to deal with the various constrained superfields in $\mathcal{N}=2$ supersymmetry. Concretely the challenge is to find the appropriate localizations of the global symmetry transformations compatible with the constraints. That this is possible at all relies on the fact that in $\mathcal{N}=2$, contrary to $\mathcal{N}=1$, (2.1) can be replaced by a stronger constraint while still localizing the super-Poincaré/superconformal transformations. This is the subject of the next subsection. In the second part, we then apply the formalism to the $\mathcal{N}=2$ vector and tensor multiplets respectively.

\section{1 $\mathcal{N}=2$ Superconformal Group}

In analogy with the $\mathcal{N}=1$ case, the constraints (2.1) can be solved in terms of an unconstrained $\mathcal{N}=2$ superfield $L_{\mathbf{i}}^{\alpha}$ as

$$
h^{\alpha \dot{\alpha}}=-\frac{2}{3} \bar{D}^{3 \dot{\alpha} \mathbf{i}} L_{\mathbf{i}}^{\alpha} \quad \text { and } \quad \bar{h}^{\alpha \dot{\alpha}}=-\frac{2}{3} D^{3 \alpha \mathbf{i}} \bar{L}_{\mathbf{i}}^{\dot{\alpha}}
$$

In the global limit, $h^{\alpha \dot{\alpha}}=\bar{h}^{\alpha \dot{\alpha}}$, the identity $\bar{D}_{\dot{\alpha} \mathbf{i}} \bar{D}_{\dot{\beta} \mathbf{j}} h^{\gamma \dot{\gamma}}=D_{\alpha \mathbf{i}} D_{\beta \mathbf{j}} h^{\gamma \dot{\gamma}}=0$ holds 36 , and a straightforward computation shows that a suitable $L_{\mathbf{i}}^{\alpha}$ is given by $L_{\mathbf{i}}^{\alpha}=-\frac{1}{12} \bar{\theta}_{\dot{\alpha} \mathbf{i}}^{3} h^{\alpha \dot{\alpha}}$. However, contrary to the $\mathcal{N}=1$ case, it is possible to replace $L_{\mathbf{i}}^{\alpha}$ by a constrained superfield without losing the global transformations. Indeed we can write

$$
L_{\mathbf{i}}^{\alpha} \equiv D^{\alpha \mathbf{j}} L_{\mathbf{j i}} \quad \text { and } \quad \bar{L}_{\mathbf{i}}^{\dot{\alpha}} \equiv-\bar{D}^{\dot{\alpha} \mathbf{j}} \bar{L}_{\mathbf{j i}}
$$

where $L_{\mathbf{i j}}$ is an unconstrained symmetric superfield [2]. The expansion of $L_{\mathbf{i j}}$ for global super-Poincaré and superconformal transformations is given in appendix A.2. This allows us to define a new scalar superfield $H$ which, like $h^{\alpha \dot{\alpha}}$, is real for superconformal transformations. Indeed we can take

$$
H \equiv \bar{D}^{\mathbf{i j}} L_{\mathrm{ij}}, \quad \bar{H} \equiv D^{\mathrm{ij}} \bar{L}_{\mathbf{i j}}
$$

Furthermore, $h^{\alpha \dot{\alpha}}$ can be expressed in terms of $H$ as

$$
h^{\alpha \dot{\alpha}}=\frac{1}{2}\left[D^{\alpha \mathbf{i}}, \bar{D}_{\mathbf{i}}^{\dot{\alpha}}\right] H \quad \text { and } \quad \bar{h}^{\alpha \dot{\alpha}}=\frac{1}{2}\left[D^{\alpha \mathbf{i}}, \bar{D}_{\mathbf{i}}^{\dot{\alpha}}\right] \bar{H} .
$$

In terms of $L_{\mathbf{i j}}$, we have

$$
\lambda_{\mathbf{i}}^{\alpha}=\frac{i}{24} \bar{D}^{4} D^{\alpha \mathbf{j}} L_{\mathbf{i j}} \quad \text { and } \quad \sigma=\frac{i}{144} \bar{D}^{4} D^{\mathbf{i j}} L_{\mathbf{i j}} .
$$

According to the prescription given in section 2, we localize the parameters of the global symmetry transformations by relaxing the reality condition $h^{\alpha \dot{\alpha}}=\bar{h}^{\alpha \dot{\alpha}}$, while maintaining the chirality preserving condition (2.1). However, in view of the discussion above we consider only those $h^{\alpha \dot{\alpha}}$ that can be written in terms of $L_{\mathbf{i j}}$ where $L_{\mathbf{i j}}$ is an arbitrary symmetric superfield.

\footnotetext{
${ }^{12}$ We checked that for super-Poincaré transformations it is even possible to integrate further by $L_{\mathbf{i} \mathbf{j}}=D_{(\mathbf{i}}^{\alpha} A_{\alpha \mathbf{j})}$ However, as this does not seem to play a role for the Superfield Noether Procedure, we do not explore this possibility further in the present work.
} 


\section{2 $\mathcal{N}=2$ Vector Multiplet}

As a first application of the general formalism we consider the Abelian vector multiplet [43]. This multiplet plays an important role for the low energy effective description of non-Abelian $\mathcal{N}=2$ Yang-Mills theory [9]. The vector multiplet is described by a chiral superfield $\mathcal{A}$ of $R$-weight -2 and dimension 1 , subject to the Bianchi constraint $D^{\mathrm{ij}} \mathcal{A}=\bar{D}^{\mathrm{ij}} \overline{\mathcal{A}}$. This constraint can be solved by $\mathcal{A}=\bar{D}^{4} D^{\mathrm{ij}} V_{\mathrm{ij}}$ where the prepotential $V_{\mathrm{ij}}$ is a real superfield.

The global superconformal transformation of $\mathcal{A}$ is given by (2.8) with $q=\frac{3}{2}$ (and, of course, $\bar{q}=0$ since $\mathcal{A}$ is chiral). Although the corresponding local transformation, i.e. when $L_{\mathbf{i j}}$ is free, does preserve the chirality of $\mathcal{A}$, it does not preserve the Bianchi constraint. Nevertheless, we can proceed by complete analogy with the $\mathcal{N}=1$ case: this suggests to generalize the transformation (3.30) of the $\mathcal{N}=1$ prepotential $V$ to

$$
\delta V_{\mathbf{i j}} \equiv-\frac{i}{48}\left(\mathcal{A} L_{\mathbf{i j}}-\overline{\mathcal{A}} \bar{L}_{\mathbf{i j}}\right)
$$

Using the definition of $\mathcal{A}$ and the constraints it satisfies, we then compute the transformation of $\mathcal{A}$. It leads to

$$
48 i \delta \mathcal{A}=\bar{D}^{4}\left(D^{\mathbf{i j}} \mathcal{A} L_{\mathbf{i j}}\right)+2 \bar{D}^{4}\left(D^{\alpha \mathbf{j}} L_{\mathbf{i j}} D_{\alpha}^{\mathbf{i}} \mathcal{A}\right)+\mathcal{A} \bar{D}^{4} D^{\mathbf{i j}} L_{\mathbf{i j}}-\bar{D}^{4}\left(\overline{\mathcal{A}} D^{\mathbf{i j}} \bar{L}_{\mathbf{i j}}\right)
$$

The Bianchi constraint enables us to write the first term in this variation as $\bar{D}^{4}\left(\bar{D}^{\mathbf{i j}} \overline{\mathcal{A}} L_{\mathbf{i j}}\right)=\bar{D}^{4}\left(\overline{\mathcal{A}} \bar{D}^{\mathbf{i j}} L_{\mathbf{i j}}\right)$. Then, using the definition of $H$ and $\bar{H}$ we end up with

$$
\delta \mathcal{A}=-\frac{i}{24} \bar{D}^{4}\left(D^{\alpha \mathbf{j}} L_{\mathbf{i j}} D_{\alpha}^{\mathbf{i}} \mathcal{A}\right)-\frac{i}{48} \bar{D}^{4} D^{\mathbf{i j}} L_{\mathbf{i j}} \mathcal{A}-\frac{i}{48} \bar{D}^{4}[(H-\bar{H}) \overline{\mathcal{A}}] .
$$

Therefore, this transformation is a suitable one as it reduces to the transformation (2.8) in the superconformal limit $H=\bar{H}$ and furthermore preserves the Bianchi constraint.

\subsubsection{Variation of the holomorphic Action}

As for the $\mathcal{N}=1$ chiral multiplet, the most general local action for the vector multiplet consists of holomorphic and a non-holomorphic terms. We first consider the holomorphic part, referring non-holomorphic terms to subsection 4.2.3.

The general holomorphic action is given by

$$
S[\mathcal{A}] \equiv \frac{1}{4 \pi} \operatorname{Im} \int d^{8} z_{+} \mathcal{F}(\mathcal{A})
$$

The classical Yang-Mills action corresponds to $\mathcal{F}(\mathcal{A}) \equiv \tau \mathcal{A}^{2}$ with $\tau \equiv \frac{\theta}{2 \pi}+i \frac{4 \pi}{g^{2}}$ where $g$ is the coupling constant and $\theta$ the vacuum-angle. For convenience, we also introduce the dual superfield $\mathcal{A}_{D} \equiv \mathcal{F}^{\prime}(\mathcal{A})$. The equations of motion are then $D^{\mathbf{i j}} \mathcal{A}_{D}=\bar{D}^{\mathbf{i j}} \overline{\mathcal{A}}_{D}$.

It is now straightforward to compute the variation of the action (4.9) under the transformation (4.8). Indeed, as

$$
\delta \mathcal{F}=-\frac{i}{24} \bar{D}^{4}\left(D^{\alpha \mathbf{j}} L_{\mathbf{i j}} D_{\alpha}^{\mathbf{i}} \mathcal{F}\right)-3 \sigma \mathcal{A} \mathcal{A}_{D}-\frac{i}{48} \bar{D}^{4}\left[(H-\bar{H}) \overline{\mathcal{A}} \mathcal{A}_{D}\right]
$$


the variation of the action is

$$
\delta S=\frac{1}{4 \pi} \operatorname{Im}\left\{-\frac{i}{24} \int d^{12} z D^{\alpha \mathbf{j}} L_{\mathbf{i j}} D_{\alpha}^{\mathbf{i}} \mathcal{F}-3 \int d^{8} z_{+} \sigma \mathcal{A} \mathcal{A}_{D}-\frac{i}{48} \int d^{12} z(H-\bar{H}) \overline{\mathcal{A}} \mathcal{A}_{D}\right\} .
$$

Then, we successively integrate by parts the first term, write it as an integral on the chiral superspace, use the chirality of $\mathcal{F}(\mathcal{A})$ and the relation (4.5) between $\sigma$ and $L_{\mathbf{i j}}$ to obtain:

$$
\delta S=\frac{1}{4 \pi} \operatorname{Im}\left\{6 \int d^{8} z_{+} \sigma\left(\mathcal{F}-\frac{1}{2} \mathcal{A} \mathcal{A}_{D}\right)-\frac{i}{48} \int d^{12} z(H-\bar{H}) \overline{\mathcal{A}} \mathcal{A}_{D}\right\} .
$$

This means that the variation of the action can be written as

$$
\delta S=i \int d^{12} z(H-\bar{H}) T-144 i \int d^{8} z_{+} \sigma \mathcal{J}+144 i \int d^{8} z_{-} \bar{\sigma} \overline{\mathcal{J}}
$$

with

$$
T=-\frac{i}{384 \pi}\left(\mathcal{A} \overline{\mathcal{A}}_{D}-\overline{\mathcal{A}} \mathcal{A}_{D}\right), \quad \mathcal{J}=\frac{1}{192 \pi}\left(\mathcal{F}-\frac{1}{2} \mathcal{A} \mathcal{A}_{D}\right)
$$

This result deserves some comments:

1) Contrary to the general situation of section 2, it is not possible to obtain the variation of the action (4.9) in terms of $h^{\alpha \dot{\alpha}}$ due to the constraints on $\mathcal{A}$.

2) Nevertheless, the invariance of the action under super-Poincaré transformations is explicit as $H=\bar{H}$ and $\sigma=\bar{\sigma}=0$ for these transformations.

Moreover, as in $\mathcal{N}=1$, the conservation equations are obtained from (4.13) by expressing $H, \bar{H}, \sigma$ and $\bar{\sigma}$ in terms of the free parameters $L_{\mathbf{i j}}$ and $\bar{L}_{\mathbf{i j}}$. This leads to

$$
D^{\mathbf{i j}} T=-i \bar{D}^{\mathbf{i j}} \overline{\mathcal{J}}
$$

3) Contrary to the situation in $\mathcal{N}=1$, there is no freedom in the definition of $T$ and $\mathcal{J}$ i.e. they are uniquely determined in terms of the constrained superfield $\mathcal{A}$.

4) It is now clear from 3) that the theory is superconformal invariant if and only if $\mathcal{J}=0$. Hence, in analogy with the $\mathcal{N}=1$ case, $\mathcal{J}$ is the superconformal anomaly and therefore our method provides a simple derivation of the anomalous superconformal 'Ward identity' first derived in [20].

\subsubsection{Central Charge}

For classical $\mathcal{N}=2$ Yang-Mills theory, the central charge was first computed in [44] by explicit evaluation of the anticommutator of the supersymmetry charges. The quantum corrected effective central charge of $\mathcal{N}=2$ supersymmetry is important because it determines the mass of the BPS states in the quantum theory. Indeed, the central charge formula for low-energy effective action of $\mathcal{N}=2$ Yang-Mills theory which was assumed in [9] contains the seeds of the duality properties of this model [10, 11]. That this assumption is correct was proved in components in 445, 46]. In general, the complete computation of central charges for effective theories in components is a rather magnificent task. On the other hand, we have seen in subsection 3.3 that the Superfield Noether Procedure leads to a simple computation even for complicated actions. This method naturally extends to the $\mathcal{N}=2$ case and as we shall now show it leads to a simple and efficient computation of the effective central charge. In order to have 
equivalence with the canonical computation 44, 45, 46] it is, however, important to work with the canonical multiplet.

As in $\mathcal{N}=1$, we first determine the supersymmetry current by taking $L_{\mathbf{i j}}=-\frac{i}{9} \varepsilon_{(\mathbf{i}}^{\alpha}\left(x^{+}\right) \theta_{\alpha \mathbf{j})} \bar{\theta}^{4}$ and $\bar{L}_{\mathbf{i j}}=\frac{2 i}{9} \varepsilon_{\mathbf{k}}^{\alpha}\left(x^{-}\right) \theta_{\alpha}^{3 \mathbf{k}} \bar{\theta}_{\mathbf{i j}}$. This leads to

$$
J_{\alpha}^{\mu \mathbf{i}}=192\left[i \sigma_{\alpha \dot{\alpha}}^{\mu} \bar{D}_{\mathbf{j}}^{\dot{\alpha}} D^{\mathbf{i j}} T-3 i \bar{\sigma}^{\mu \dot{\alpha} \beta} \bar{D}_{\dot{\alpha}}^{\mathbf{i}} D_{\alpha \beta} T-12 \partial^{\mu} D_{\alpha}^{\mathbf{i}} T+a \sigma_{\alpha}^{\mu \nu \beta} \partial_{\nu} D_{\beta}^{\mathbf{i}} T\right] \mid,
$$

where the term parametrized by a corresponds to an improvement term. Note that the first term in the r.h.s.. of (4.16) is equal on-shell to $192 \sigma_{\alpha \dot{\alpha}}^{\mu} \bar{D}^{3 \dot{\alpha} \mathbf{i}} \overline{\mathcal{J}}$. As the second term in the r.h.s of (4.16) is traceless, the trace of $J_{\alpha}^{\mu \mathbf{i}}$ is simply given by

$$
\left(\bar{\sigma}^{\mu} J_{\mu}\right)^{\dot{\alpha} \mathbf{i}}=192\left[-4 \bar{D}^{3 \dot{\alpha} \mathbf{i}} \overline{\mathcal{J}}-\frac{3}{2}(8+a) \partial^{\dot{\alpha} \alpha} D_{\alpha}^{\mathbf{i}} T\right] \mid .
$$

Therefore, this trace vanishes when $\mathcal{J}=0$ (superconformal case) and $a=-8$.

Next, we give the relevant part of the variation of $J_{\alpha}^{\mu \mathbf{i}}$ referring to appendix B.1 for details of this computation:

$$
\delta_{\alpha \mathbf{i}} J_{\beta \mathbf{j}}^{\mu}=\partial_{\nu}\left\{24 i \varepsilon_{\alpha \beta} \varepsilon_{\mathbf{i j}} Z^{\mu \nu}+96(a+24)\left[\varepsilon_{\mathbf{i j}} \sigma_{(\alpha}^{\mu \nu \gamma} D_{\beta) \gamma} T\left|+\sigma_{\alpha \beta}^{\mu \nu} D_{\mathbf{i j}} T\right|\right]\right\}
$$

where we have defined

$$
Z^{\mu \nu} \equiv 96\left[\bar{\sigma}_{\dot{\alpha} \dot{\beta}}^{\mu \nu} \bar{D}^{\dot{\alpha} \dot{\beta}} \overline{\mathcal{J}}-i\left(\frac{1}{2}-\frac{a}{48}\right) \sigma_{\alpha \beta}^{\mu \nu} D^{\alpha \beta} T\right] \mid .
$$

One way to fix the value of $a$ corresponding to the canonical current is to go to components in the particular case of classical Yang-Mills theory, where $\mathcal{F}(\mathcal{A})$ is quadratic. This leads to $a=-24$. However, it is also possible to determine this value at the superspace level, for any $\mathcal{F}$, by comparing the variation (4.18) of the supersymmetry current and the supersymmetry algebra

$$
\left\{Q_{\alpha \mathbf{i}}, Q_{\beta \mathbf{j}}\right\}=\frac{1}{4} \varepsilon_{\mathbf{i j}} \varepsilon_{\alpha \beta} Z+\sigma_{\alpha \beta}^{0 k} \Lambda_{k(\mathbf{i j})} .
$$

Indeed, this Poisson algebra allows only terms having the same symmetry properties in the $S U(2)_{R}$ and spinor indices. On the other hand as $\left\{Q_{\alpha \mathbf{i}}, Q_{\beta \mathbf{j}}\right\}=\int d^{3} x \delta_{\alpha \mathbf{i}} J_{\beta \mathbf{j}}^{0}$, it is clear that the r.h.s. of equation (4.18) has the required symmetry only when $a=-24$. Note that this implies the vanishing of $\Lambda_{k(\mathbf{i j})}$. According to the previous discussion, the central charge $Z$ is then given by

$$
Z=\int d^{3} x \delta^{\alpha \mathbf{i}} J_{\alpha \mathbf{i}}^{0}=96 \int d^{3} x \partial_{i} Z^{0 i}
$$

where $Z^{0 i}$ is given for $a=-24$ by

$$
Z^{0 i}=96 i\left[\bar{\sigma}_{\dot{\alpha} \dot{\beta}}^{0 i} \bar{D}^{\dot{\alpha} \dot{\beta}} \overline{\mathcal{J}}-i \sigma_{\alpha \beta}^{0 i} D^{\alpha \beta} T\right] \mid
$$

Note that this result is universal in the sense that this formula for the center is valid for any theory whose variation of the action takes the form (4.13).

Using the particular expressions (4.14) of $T$ and $\overline{\mathcal{J}}$, we end up with

$$
Z^{0 i}=\frac{i}{4 \pi} \overline{\mathcal{A}}\left\{\sigma_{\alpha \beta}^{0 i} D^{\alpha \beta} \mathcal{A}_{D}-\bar{\sigma}_{\dot{\alpha} \dot{\beta}}^{0 i} \bar{D}^{\dot{\alpha} \dot{\beta}} \overline{\mathcal{A}}_{D}\right\}\left|-\frac{i}{4 \pi} \overline{\mathcal{A}}_{D}\left\{\sigma_{\alpha \beta}^{0 i} D^{\alpha \beta} \mathcal{A}-\bar{\sigma}_{\dot{\alpha} \dot{\beta}}^{0 i} \bar{D}^{\dot{\alpha} \dot{\beta}} \overline{\mathcal{A}}\right\}\right|
$$


This result, which is the expression of the central charge in superspace exhibits manifestly the duality between $\mathcal{A}$ and $\mathcal{A}_{D}$. However, to complete the calculation we need to identify the r.h.s. of (4.23) with the electric and magnetic charges.

To identify the magnetic charge we use that $\left[3 D_{\alpha \beta} \mathcal{A} \mid \equiv \frac{1}{2 \sqrt{3}} F_{\alpha \beta}\right.$, where $F_{\alpha \beta} \equiv$ $F_{\mu \nu} \sigma_{\alpha \beta}^{\mu \nu}$ and $B^{i} \equiv \frac{1}{2} \varepsilon^{o i j k} F_{j k}$. It follows then that

$$
B^{i}=-i \sqrt{3}\left[\sigma_{\alpha \beta}^{0 i} D^{\alpha \beta} \mathcal{A}-\bar{\sigma}_{\dot{\alpha} \dot{\beta}}^{0 i} \bar{D}^{\dot{\alpha} \dot{\beta}} \overline{\mathcal{A}}\right] \mid
$$

Next we determine the conjugate momentum $\Pi^{i}$ of the gauge field. In general, it is not straightforward to determine the conjugate momentum of a field in superspace. However, we proceed by using that $\Pi^{i}$ can be extracted from the Gauss law $\partial_{i} \Pi^{i}=0$ which is an equation of motion. More precisely, the gauge field appears in the action only via the field strength (in superspace language, this is reflected by the fact that the superfield $\mathcal{A}$ is a gauge invariant object). Therefore, we have the sequence of equalities:

$$
\partial_{i} \Pi^{i}=\partial_{i} \frac{\partial L}{\partial\left(\partial_{0} A_{i}\right)}=-\partial_{i} \frac{\partial L}{\partial\left(\partial_{i} A_{0}\right)}=\frac{\delta S}{\delta A_{0}}
$$

which is just expressing the fact that Gauss law is equivalent to $\frac{\delta S}{\delta A_{0}}$. To obtain Gauss law in superspace we then proceed in two steps. First we compute the fundamental derivative

$$
\frac{\delta}{\delta A_{0}(y)}\left(D_{\alpha \beta} \mathcal{A} \mid(x)\right)=-\frac{1}{\sqrt{3}} \sigma_{\alpha \beta}^{0 i} \partial_{x i} \delta(x-y) .
$$

Then we identify the terms in the action containing $D_{\alpha \beta} A$. Now, since

$$
D^{4} \mathcal{F}(\mathcal{A})=\mathcal{F}^{\prime} D^{4} \mathcal{A}+3 D^{\alpha \mathbf{i}} \mathcal{F}^{\prime} D_{\alpha \mathbf{i}}^{3} \mathcal{A}+\frac{3}{2} D^{\mathbf{i} \mathbf{j}} \mathcal{F}^{\prime} D_{\mathbf{i j}} \mathcal{A}-\frac{3}{2} D^{\alpha \beta} \mathcal{F}^{\prime} D_{\alpha \beta} \mathcal{A}-D^{3 \alpha \mathbf{i}} \mathcal{F}^{\prime} D_{\alpha \mathbf{i}} \mathcal{A}
$$

the terms we are looking for are

$$
-\frac{3}{8 \pi} \operatorname{Im} \int d^{4} x D^{\alpha \beta} \mathcal{F}^{\prime} D_{\alpha \beta} \mathcal{A} \mid
$$

Differentiating with respect to $A_{0}$ and using (4.26) we then end up with

$$
\frac{\delta S}{\delta A_{0}}=-\frac{\sqrt{3}}{8 \pi} \partial_{i} \operatorname{Im}\left[\sigma_{\alpha \beta}^{0 i}\left(D^{\alpha \beta} \mathcal{F}^{\prime}+\mathcal{F}^{\prime \prime} D^{\alpha \beta} \mathcal{A}\right)\right] \mid .
$$

Thus we have

$$
\Pi^{i}=-\frac{\sqrt{3}}{8 \pi} \operatorname{Im}\left[\sigma_{\alpha \beta}^{0 i}\left(D^{\alpha \beta} \mathcal{F}^{\prime}+\mathcal{F}^{\prime \prime} D^{\alpha \beta} \mathcal{A}\right)\right] \mid .
$$

We now consider the phase space for which $\phi \equiv \mathcal{A} \mid$ goes to a constant at infinity and where the fermions $D^{\alpha \mathbf{i}} \mathcal{A} \mid$ decrease sufficiently fast enough such that

$$
\mathcal{F}^{\prime \prime} D^{\alpha \beta} \mathcal{A}\left|=D^{\alpha \beta} \mathcal{F}^{\prime}\right|-\mathcal{F}^{\prime \prime \prime} D^{\alpha \mathbf{i}} \mathcal{A} D_{\mathbf{i}}^{\beta} \mathcal{A}\left|\stackrel{|x| \rightarrow \infty}{\longrightarrow} D^{\alpha \beta} \mathcal{F}^{\prime}\right|
$$

\footnotetext{
${ }^{13}$ For classical Yang-Mills theory where $\mathcal{F}(\mathcal{A})=i \frac{4 \pi}{g^{2}} \mathcal{A}^{2}$, this definition leads to the usual normalization $-\frac{1}{4 g^{2}} F^{\mu \nu} F_{\mu \nu}$.
} 
Therefore, we have, at infinity, $\Pi^{i}=-\frac{\sqrt{3}}{4 \pi} \operatorname{Im} \sigma_{\alpha \beta}^{0 i} D^{\alpha \beta} \mathcal{A}_{D} \mid$, i.e.

$$
\Pi^{i}=i \frac{\sqrt{3}}{8 \pi}\left[\sigma_{\alpha \beta}^{0 i} D^{\alpha \beta} \mathcal{A}_{D}-\bar{\sigma}_{\dot{\alpha} \dot{\beta}}^{0 i} \bar{D}^{\dot{\alpha} \dot{\beta}} \overline{\mathcal{A}}_{D}\right] \mid .
$$

Finally, combining the expressions (4.24) and (4.32) respectively for the magnetic and electric fields, the result for the central charge (4.23) can be rewritten as

$$
Z^{0 i} \stackrel{|x| \rightarrow \infty}{\longrightarrow} \frac{2}{\sqrt{3}}\left\{\overline{\mathcal{A}} \Pi^{i}+\frac{1}{8 \pi} \overline{\mathcal{A}}_{D} B^{i}\right\} \mid
$$

which corresponds to the result found in 45.

\subsubsection{Non-holomorphic Action}

We now consider a non-holomorphic, local action for the vector multiplet

$$
S[\mathcal{A}] \equiv \int d^{12} z \mathcal{H}(\mathcal{A}, \overline{\mathcal{A}})
$$

To compute the corresponding $T$ and $\mathcal{J}$, we start from the following form of (4.8):

$$
\delta \mathcal{A}=\frac{1}{2} h^{\alpha \dot{\alpha}} \partial_{\alpha \dot{\alpha}} \mathcal{A}+\lambda^{\alpha \mathbf{i}} D_{\alpha \mathbf{i}} \mathcal{A}-3 \sigma \mathcal{A}-\frac{i}{48} \bar{D}^{4}((H-\bar{H}) \overline{\mathcal{A}}) .
$$

The contributions from the last two terms to $T$ and $\mathcal{J}$ are immediately obtained. Let us so concentrate on the first two terms. We use the relations (2.3) to obtain

$$
\begin{aligned}
\delta S & =\int d^{12} z\left\{h^{\alpha \dot{\alpha}}\left(\frac{1}{2} \mathcal{H}_{\mathcal{A}} \partial_{\alpha \dot{\alpha}} A+\frac{i}{8} \bar{D}_{\dot{\alpha}}^{\mathbf{i}} D_{\alpha \mathbf{i}} \mathcal{H}\right)+\bar{h}^{\alpha \dot{\alpha}}\left(\frac{1}{2} \mathcal{H}_{\overline{\mathcal{A}}} \partial_{\alpha \dot{\alpha}} \overline{\mathcal{A}}-\frac{i}{8} D^{\alpha \mathbf{i}} \bar{D}_{\mathbf{i}}^{\dot{\alpha}} \mathcal{H}\right)\right\} \\
& =\int d^{12} z \frac{1}{2}\left(h^{\alpha \dot{\alpha}}-\bar{h}^{\alpha \dot{\alpha}}\right)\left(\frac{1}{2} \mathcal{H}_{\mathcal{A}} \partial_{\alpha \dot{\alpha}} A-\frac{1}{2} \mathcal{H}_{\overline{\mathcal{A}}} \partial_{\alpha \dot{\alpha}} \overline{\mathcal{A}}-\frac{i}{8}\left[D_{\alpha \mathbf{i}}, \bar{D}_{\dot{\alpha}}^{\mathbf{i}}\right] \mathcal{H}\right) \\
& =\int d^{12} z \frac{1}{2}\left(h^{\alpha \dot{\alpha}}-\bar{h}^{\alpha \dot{\alpha}}\right)\left(\frac{i}{4} \mathcal{H}_{\mathcal{A} \overline{\mathcal{A}}} D_{\alpha \mathbf{i}} \mathcal{A} \bar{D}_{\dot{\alpha}}^{\mathbf{i}} \overline{\mathcal{A}}\right) .
\end{aligned}
$$

We conclude by using the relation (4.4) between $h^{\alpha \dot{\alpha}}$ and $H$ and by integrating by parts. Adding up all the contributions finally leads to

$$
\begin{aligned}
T & =-\frac{1}{48}\left[\overline{\mathcal{A}} \bar{D}^{4} \mathcal{H}_{\mathcal{A}}+\mathcal{A} D^{4} \mathcal{H}_{\overline{\mathcal{A}}}+3\left[D^{\alpha \mathbf{i}}, \bar{D}_{\mathbf{i}}^{\dot{\alpha}}\right]\left(\mathcal{H}_{\mathcal{A} \overline{\mathcal{A}}} D_{\alpha}^{\mathbf{j}} \mathcal{A} \bar{D}_{\dot{\alpha} \mathbf{j}} \overline{\mathcal{A}}\right)\right] \\
\mathcal{J} & =-\frac{i}{48} \mathcal{A} \bar{D}^{4} \mathcal{H}_{\mathcal{A}}
\end{aligned}
$$

We finish this section by showing that the non-holomorphic part of the action does not contribute to the center. We denote generically by a subscript $h$, holomorphic terms and by a subscript $n . h$., non-holomorphic ones.

Among the diverse terms that appear in the expression (4.22) for the center $Z_{n . h}^{0 i}$, we concentrate on the ones which fall off slowest at infinity. Let us take the example of $\bar{D}^{\dot{\alpha} \dot{\beta}} \overline{\mathcal{J}} \propto \bar{D}^{\dot{\alpha} \dot{\beta}}\left(\overline{\mathcal{A}} D^{4} \mathcal{H}_{\overline{\mathcal{A}}}\right)$. Remember that the dimension of $\mathcal{A}$ is -1 . Furthermore, as 
$\mathcal{A} \mid$ goes to a constant at infinity, all the derivatives of $\mathcal{H}$ share this property. Therefore, the term that decreases the least is the one where as less as possible derivatives act on $\mathcal{H}$ namely $\overline{\mathcal{A}} \mathcal{H}_{\mathcal{A} \overline{\mathcal{A}}}\left(\bar{D}^{\dot{\alpha} \dot{\beta}} D^{4} \mathcal{A}\right)$. Clearly, this term decreases sufficiently fast enough at infinity, and thus, this holds similarly for all the other terms. As a consequence, there is no contribution to the center from $\overline{\mathcal{J}}$ and, for the same reasons, from $T$ such that $Z_{n . h .}^{0 i} \stackrel{|x| \rightarrow \infty}{\longrightarrow} 0$. However, in the same way, by computing the Gauss law, it is easy to prove that at infinity, the non-holomorphic terms do not contribute to $\Pi^{i}$. Thus, in a theory where both holomorphic and non-holomorphic terms are present, we have, on one hand, $\Pi^{i}=\Pi_{h .}^{i}+\Pi_{n . h .}^{i} \stackrel{|x| \rightarrow \infty}{\longrightarrow} \Pi_{h .}^{i}$ and on the other hand $Z^{0 i}=Z_{h .}^{0 i}+Z_{n . h .}^{0 i} \stackrel{|x| \rightarrow \infty}{\longrightarrow} Z_{h .}^{0 i}$. Therefore,

$$
Z^{0 i} \stackrel{|x| \rightarrow \infty}{\longrightarrow} \frac{2}{\sqrt{3}}\left\{\overline{\mathcal{A}} \Pi_{h .}^{i}+\frac{1}{8 \pi} \overline{\mathcal{A}}_{D} B^{i}\right\}\left|=\frac{2}{\sqrt{3}}\left\{\overline{\mathcal{A}} \Pi^{i}+\frac{1}{8 \pi} \overline{\mathcal{A}}_{D} B^{i}\right\}\right| .
$$

This proves that the central charge is entirely determined by the holomorphic part of the action.

\section{3 $\mathcal{N}=2$ Tensor Multiplet}

The second example of a $\mathcal{N}=2$ theory with off-shell superfield formulation we consider is the tensor multiplet [50, 51]. This multiplet is described by a chiral field $\Phi$ of dimension 1 and $R$-weight -2 and classical action

$$
S[\Phi] \equiv \int d^{4} x d^{8} \theta \Phi \bar{\Phi}+24 \int d^{4} x d^{4} \theta \Phi\left(m^{2}-\square\right) \Phi+24 \int d^{4} x d^{4} \bar{\theta} \bar{\Phi}\left(m^{2}-\square\right) \bar{\Phi} .
$$

For $m=0$, this action is invariant under the gauge transformation $\delta \Phi \equiv \bar{D}^{4} D^{\mathbf{i j}} K_{\mathbf{i j}}$ where $K_{\mathrm{ij}}$ is real. The invariant "field strength" tensor is given by

$$
F_{\mathrm{ij}} \equiv i\left(D_{\mathrm{ij}} \Phi-\bar{D}_{\mathrm{ij}} \bar{\Phi}\right)
$$

which is the analogue of eq.(3.38) for the $\mathcal{N}=1$ tensor. It satisfies the following properties:

$$
\begin{aligned}
\bar{D}_{\dot{\alpha}}^{(\mathbf{i}} F^{\mathbf{j k})}=0 & \text { (completely symmetric) }, \\
\bar{D}_{\dot{\alpha} \mathbf{i}} F_{\mathbf{j} \mathbf{k}}=\frac{2}{3} \varepsilon_{\mathbf{i}(\mathbf{j}} \bar{D}_{\dot{\alpha}}^{\mathbf{1}} F_{\mathbf{k}) \mathbf{l}}, \quad & \bar{D}_{\mathbf{i j}} F_{\mathbf{k} \mathbf{l}}=\frac{1}{3} \varepsilon_{\mathbf{i}(\mathbf{k}} \varepsilon_{\mathbf{j} \mathbf{l})} \bar{D}^{\mathbf{m n}} F_{\mathbf{m n}} .
\end{aligned}
$$

Finally, $\bar{D}_{\mathbf{i j}} F^{\mathbf{i j}}$ is chiral and so is $\bar{D}_{\mathbf{i j}} F_{\mathbf{k l}}$ as a consequence of (4.42).

The equation of motion can be written in the two equivalent forms

$$
\bar{D}^{4} \bar{\Phi}-48\left(\square-m^{2}\right) \Phi=0 \quad \Longleftrightarrow \quad i \bar{D}^{\mathbf{i j}} F_{\mathbf{i j}}+48 m^{2} \Phi=0 .
$$

\subsubsection{Supercurrent}

The variation of $\Phi$ under superconformal transformations is given by eq. (2.8) with $q=\frac{3}{2}($ and $\bar{q}=0)$, i.e.

$$
\delta \Phi=-\frac{i}{48} \bar{D}^{4} D^{\mathbf{i j}}\left(L_{\mathbf{i j}} \Phi\right)+\frac{i}{48} \bar{D}^{4}\left(L_{\mathbf{i j}} D^{\mathbf{i j}} \Phi\right) .
$$


As in $\mathcal{N}=1$, it is convenient to add to the local corresponding transformation the term $-\frac{i}{48} \bar{D}^{4}((H-\bar{H}) \bar{\Phi})$ that vanishes in the global limit. This leads to

$$
\delta \Phi=\frac{1}{48} \bar{D}^{4}\left(L_{\mathbf{i j}} F^{\mathbf{i j}}\right)-\frac{i}{48} \bar{D}^{4} D^{\mathbf{i j}}\left(L_{\mathbf{i j}} \Phi-\bar{L}_{\mathbf{i j}} \bar{\Phi}\right)
$$

which is of the same form as for the vector multiplet. The advantage of this form is that, for $m=0$, the second term is in fact a gauge transformation and can be ignored. To continue we then treat the variation of the mass term, $S_{m}$, and of the gauge invariant part, $S_{g}$ separately. The action $S_{m}$ is similar to the $\mathcal{N}=2$ classical Yang-Mills action, i.e. the action (4.9) with $\mathcal{F}(\mathcal{A}) \equiv i 192 \pi m^{2} \mathcal{A}^{2}$. It follows then from (4.13)-(4.14) that

$$
\delta S_{m}=-2 i m^{2} \int(H-\bar{H}) \Phi \bar{\Phi}
$$

$S_{g}$ being gauge invariant, we can compute its variation by taking only into account the first term in the variation given by eq.(4.45). This leads to

$$
\begin{aligned}
\delta S_{g} & =\int d^{4} x d^{4} \theta \delta \Phi \frac{\delta S_{g}}{\delta \Phi}+\text { c.c. }=\int d^{4} x d^{8} \theta\left(\frac{1}{48} L_{\mathbf{i j}} F^{\mathbf{i j}}\right) \frac{\delta S_{g}}{\delta \Phi}+\text { c.c. } \\
& =\frac{i}{48} \int L_{\mathbf{i j}} F^{\mathbf{i j}} \bar{D}^{\mathbf{k} \mathbf{l}} F_{\mathbf{k} \mathbf{l}}+\text { c.c. }
\end{aligned}
$$

Naively, one might expect that this variation should be proportional to $H-\bar{H}$ and $\sigma$. However, it turns out that it is impossible to write it in this form. Nevertheless, it can be brought into the suggestive form

$$
\delta S_{g}=\frac{i}{48}\left[\int(H-\bar{H}) F^{\mathbf{i j}} F_{\mathbf{i j}}+\frac{3}{10}(H-\bar{H})_{\mathbf{i j k} \mathbf{l}} F^{\mathbf{i j}} F^{\mathbf{k l}}\right]
$$

where

$$
H_{\mathbf{i j k l}} \equiv \bar{D}_{(\mathbf{i j}} L_{\mathbf{k l})} \quad \text { (completely symmetric) }
$$

This result relies on the identity

$$
F_{\mathbf{i j}} \bar{D}^{\mathbf{k l}} F_{\mathbf{k} \mathbf{l}}=\bar{D}_{\mathbf{i j}}\left(F^{\mathbf{k} \mathbf{l}} F_{\mathbf{k l}}\right)+\frac{3}{10} \bar{D}^{\mathbf{k l}}\left(F_{(\mathbf{i j}} F_{\mathbf{k l})}\right)
$$

which in turn can be obtained from the properties (4.42) of the field strength. More precisely, one shows that

$$
\begin{aligned}
\bar{D}_{\mathbf{i j}}\left(F^{\mathbf{k} \mathbf{l}} F_{\mathbf{k} \mathbf{l}}\right) & =\frac{2}{3} F_{\mathbf{i} \mathbf{j}} \bar{D}^{\mathbf{k} \mathbf{l}} F_{\mathbf{k} \mathbf{l}}-\frac{4}{9} \bar{D}_{\dot{\alpha}}^{\mathbf{k}} F_{(\mathbf{i k}} \bar{D}^{\dot{\alpha} \mathbf{l}} F_{\mathbf{j}) \mathbf{l}} \\
\bar{D}^{\mathbf{k} \mathbf{l}}\left(F_{(\mathbf{i j}} F_{\mathbf{k} \mathbf{l})}\right) & =\frac{10}{9} F_{\mathbf{i} \mathbf{j}} \bar{D}^{\mathbf{k} \mathbf{l}} F_{\mathbf{k} \mathbf{l}}+\frac{40}{27} \bar{D}_{\dot{\alpha}}^{\mathbf{k}} F_{(\mathbf{i} \mathbf{k}} \bar{D}^{\dot{\alpha} \mathbf{l}} F_{\mathbf{j}) \mathbf{l}}
\end{aligned}
$$

We then get the relation (4.50) as a consequence of (4.51).

Taking the sum of (4.46) and of (4.48), we end up with the variation of the total action for the tensor multiplet

$$
\delta S=\frac{i}{48} \int d^{12} z(H-\bar{H})\left(F^{\mathbf{i j}} F_{\mathbf{i j}}-96 m^{2} \Phi \bar{\Phi}\right)+\frac{3}{10}(H-\bar{H})_{\mathbf{i j k l}} F^{\mathbf{i j}} F^{\mathbf{k l}} .
$$


Therefore we define

$$
T \equiv \frac{1}{48}\left(F^{\mathbf{i j}} F_{\mathbf{i j}}-96 m^{2} \Phi \bar{\Phi}\right) \quad \text { and } \quad T^{\mathbf{i j k} \mathbf{l}} \equiv \frac{1}{160} F^{(\mathbf{i j}} F^{\mathbf{k l})},
$$

where $T^{\mathrm{ijkl}}$ is completely symmetric. Indeed, it follows from the analysis of subsection 4.1, and from the expression (A.7) of $L_{\mathrm{ij}}$ for superconformal transformations, that for $\mathcal{N}=2$ super-Poincaré and $U(1)_{R}$ transformations, $H$ and $H_{\mathrm{ijkl}}$ are both real. Thus, eq.(4.52) makes the invariance of the action under these transformations explicit and the corresponding conserved currents are expressed as components of $T$ and $T^{\mathrm{ijkl}}$ in analogy with the previous examples. For the $S U(2)_{R}$-transformations and the corresponding current, the situation is more subtle. We refer the proof for this case to subsection 6.3. Finally, if we define $\tau^{\mathrm{ij}} \equiv-\bar{D}_{\mathbf{k l}} T^{\mathrm{ijkl}}$, the conservation equation following from (4.52) can be written as

$$
\bar{D}^{\mathbf{i j}} T=\tau^{\mathbf{i j}}=-\bar{D}_{\mathbf{k l}} T^{\mathbf{i j k} \mathbf{l}} .
$$

This form of the conservation equation, which was proposed in [52], is similar to that proposed in [53] except from the fact that here $\tau^{\mathrm{ij}}$ is not real.

\subsubsection{Central Charge}

We end this section with the computation of the center for the $\mathcal{N}=2$ tensor multiplet. As for the vector multiplet, we first determine the supersymmetry current. A short computation shows that $T^{\mathrm{ijk} \mathbf{l}}$ contributes to $J_{\alpha}^{\mu \mathrm{i}}$ a term

$$
768 i \sigma_{\alpha \dot{\alpha}}^{\mu} \bar{D}_{\mathbf{j}}^{\dot{\alpha}} D_{\mathbf{k l}} T^{\mathbf{i j k l}}=-768 i \sigma_{\alpha \dot{\alpha}}^{\mu} \bar{D}_{\mathbf{j}}^{\dot{\alpha}} D^{\mathbf{i j}} T
$$

where we have used the equation of conservation. This means that $J_{\alpha}^{\mu \mathrm{i}}$ can be expressed in terms of $T$ only. This is due to the fact that, for supersymmetry transformations, it is possible to localize $L_{\mathrm{ij}}$ such that $H_{\mathrm{ijk}}=\bar{H}_{\mathrm{ijk}}$ is still satisfied. Th Taking all the contributions we end up with

$$
J_{\alpha}^{\mu \mathbf{i}}=192\left[-3 i \sigma_{\alpha \dot{\alpha}}^{\mu} \bar{D}_{\mathbf{j}}^{\dot{\alpha}} D^{\mathbf{i j}} T-3 i \bar{\sigma}^{\mu \dot{\alpha} \beta} \bar{D}_{\dot{\alpha}}^{\mathbf{i}} D_{\alpha \beta} T-12 \partial^{\mu} D_{\alpha}^{\mathbf{i}} T+a \sigma_{\alpha}^{\mu \nu \beta} \partial_{\nu} D_{\beta}^{\mathbf{i}} T\right] \mid .
$$

Note that as a consequence of the conservation equation (4.54), we have $D_{\alpha \mathbf{i}}^{3} T=0$ on-shell. This identity is useful for the following reason. As explained for the vector, to fix the value of the coefficient $a$ corresponding to the canonical case and to determine the center, we have to compute the symmetric or antisymmetric part of $D_{\alpha}^{\mathbf{i}} J_{\beta \mathbf{i}}^{0}$ respectively. However, the corresponding variation of the first term in the r.h.s. of (4.56) is proportional to $D_{\alpha}^{\mathbf{i}} \bar{D}^{\dot{\alpha} \mathbf{j}} D_{\mathbf{i j}} T \propto \bar{D}^{\dot{\alpha} \mathbf{j}} D_{\alpha \mathbf{j}}^{3} T=0$. Therefore, we can ignore this term for our purpose.

Let us now consider the variation of the second term in the r.h.s. of (4.56). Using the algebraic identity (B.3) and the fact that $D^{3} T$ vanishes on-shell, we get

$$
D_{\alpha}^{\mathbf{i}}\left[-3 i \bar{\sigma}^{0 \dot{\alpha} \gamma} \bar{D}_{\dot{\alpha} \mathbf{i}} D_{\beta \gamma} T\right]=-24\left[\sigma_{\alpha}^{\mu 0} \gamma \partial_{\mu} D_{\beta \gamma} T-\frac{1}{2} \partial^{0} D_{\alpha \beta} T\right] .
$$

\footnotetext{
${ }^{14}$ This choice of $L_{\mathbf{i j}}$ differs from the one made in section 4.2 .2 by terms proportional to the derivative of $\varepsilon_{\mathbf{i}}^{\alpha}$. The corresponding currents are equal on-shell (see also remark in section 2).
} 
Finally, the variations of the last two terms in the r.h.s. of (4.56) can be easily read from the results $(\overline{B .6})$ and $(\overline{B .8})$. Here we give the result. First, as in the case of the vector multiplet, the canonical supersymmetry current corresponds to $a=-24$. Then, we get $Z=96 \int d^{3} x \partial_{i} Z^{0 i}$ with

$$
Z^{0 i}=96 \sigma_{\alpha \beta}^{0 i} D^{\alpha \beta} T \mid
$$

That is we recover the universal formula (4.22) with $\mathcal{J}=0$. Using the expression (4.53) of $T$ we then end up with

$$
Z^{0 i}=2 \sigma_{\alpha \beta}^{0 i} D^{\alpha \beta}\left(F^{\mathbf{i j}} F_{\mathbf{i j}}-96 m^{2} \Phi \bar{\Phi}\right) \mid
$$

However,

$$
D_{\alpha \beta} F_{\mathbf{i j}}=i\left(D_{\alpha \beta} D_{\mathbf{i j}} \Phi-D_{\alpha \beta} \bar{D}_{\mathbf{i j}} \bar{\Phi}\right)=8 \partial_{(\alpha \dot{\alpha}} D_{\beta)(\mathbf{i}} \bar{D}_{\mathbf{j})}^{\dot{\alpha}} \bar{\Phi}=0
$$

where we have used the fact that $D_{\alpha \beta} D_{\mathbf{i j}}=0$, the result (D.4) of appendix D.2 and the anti-chirality of $\bar{\Phi}$. As a consequence, the first term in the center (4.59) receives contribution only from $(D F)^{2}$ i.e. from fermions. Therefore, two cases have to be considered.

- $m \neq 0$ : in that case all the fields decrease sufficiently fast enough at infinity such that the center vanishes.

- $m=0$ : as there are only contributions from the fermions that decrease sufficiently fast enough at infinity, the center vanishes also in that case.

\section{$5 \quad$ Linearized Supergravity}

It is well known that the on-shell multiplet of Noether currents of supersymmetric matter theories can be used to construct off-shell Supergravities [12], at least at the linearized level. The purpose of this section is to discuss some elements of constructing supergravities directly on the superfield level using the Superfield Noether Procedure developed in this paper.

Coupling matter to gravity means gauging the super-Poincaré transformations. As explained in section 2, super-Poincaré transformations are parametrized by $h^{\alpha \dot{\alpha}}=\bar{h}^{\alpha \dot{\alpha}}$ and

$$
\sigma=\bar{\sigma}=\Lambda_{\mathbf{j}}^{\mathbf{i}}=0
$$

On the other hand, we recall from (2.11) and (2.12) that the variation of the matter action under an arbitrary local transformation is of the general form

$$
\begin{aligned}
\delta S\left[O^{A \mathbf{J}}\right]= & \frac{i}{16} \int d^{4+4 \mathcal{N}} z\left\{\left(h^{\alpha \dot{\alpha}}-\bar{h}^{\alpha \dot{\alpha}}\right) T_{\alpha \dot{\alpha}}+i\left(h^{\alpha \dot{\alpha}}+\bar{h}^{\alpha \dot{\alpha}}\right) K_{\alpha \dot{\alpha}}\right\} \\
& -\frac{1}{2} \int d^{4+2 \mathcal{N}} z_{+} \sigma J-\frac{1}{2} \int d^{4+2 \mathcal{N}} z_{-} \bar{\sigma} \bar{J}
\end{aligned}
$$

where

$$
K_{\alpha \dot{\alpha}}=-\frac{i}{4}\left[D_{\alpha}^{\mathbf{i}}, \bar{D}_{\mathbf{i} \dot{\alpha}}\right](X-\bar{X})-\frac{\mathcal{N}}{2} \partial_{\alpha \dot{\alpha}}(X+\bar{X}) .
$$


To construct an invariant action at the linear level, one follows the standard procedure of coupling the currents to potentials. Concretely we introduce the real superpotentials $H^{\alpha \dot{\alpha}}, B, C$, as well as a chiral potential $\Omega$ and add the terms

$\frac{i}{16} \int d^{4+4 \mathcal{N}} z\left\{H^{\alpha \dot{\alpha}} T_{\alpha \dot{\alpha}}+B(X+\bar{X})+C(X-\bar{X})\right\}-\frac{1}{2} \int d^{2+2 \mathcal{N}} z_{+} \Omega J-\frac{1}{2} \int d^{2+2 \mathcal{N}} z_{-} \bar{\Omega} \bar{J}$

to the action. The supergravity potentials must then transform like

$$
\begin{gathered}
\delta H^{\alpha \dot{\alpha}} \equiv-\left(h^{\alpha \dot{\alpha}}-\bar{h}^{\alpha \dot{\alpha}}\right), \\
\delta B \equiv-\frac{i \mathcal{N}}{2} \partial_{\alpha \dot{\alpha}}\left(h^{\alpha \dot{\alpha}}+\bar{h}^{\alpha \dot{\alpha}}\right), \quad \delta C \equiv-\frac{1}{4}\left[D_{\alpha}^{\mathbf{i}}, \bar{D}_{\mathbf{i} \dot{\alpha}}\right]\left(h^{\alpha \dot{\alpha}}+\bar{h}^{\alpha \dot{\alpha}}\right), \\
\delta \Omega \equiv-\sigma .
\end{gathered}
$$

By construction, the action obtained in this way has a larger invariance than the superdiffeomorphisms. In particular, it is invariant under linearized Weyl transformations

$$
h^{\alpha \dot{\alpha}} \equiv i \frac{12}{\mathcal{N}} \theta_{\dot{\mathbf{i}}}^{\alpha} \bar{\theta}^{\dot{\alpha} \mathbf{i}} \sigma\left(x^{+}\right), \quad \bar{h}^{\alpha \dot{\alpha}} \equiv-i \frac{12}{\mathcal{N}} \theta_{\mathbf{i}}^{\alpha} \bar{\theta}^{\dot{\alpha} \mathbf{i}} \bar{\sigma}\left(x^{-}\right) .
$$

Indeed, $h^{\alpha \dot{\alpha}}$ satisfies the chirality preserving constraint $\bar{D}_{\dot{\mathbf{i}}}^{(\dot{\beta}} h^{\alpha \dot{\alpha})}=0$. On the other hand, the metric $g^{\mu \nu}$, which is proportional to $\bar{\sigma}^{(\mu \dot{\alpha} \alpha} \bar{\sigma}^{\nu) \dot{\beta} \beta}\left[D_{\alpha}^{\mathbf{i}}, \bar{D}_{\dot{\alpha} \mathbf{i}}\right] H_{\beta \dot{\beta}} \mid$, transforms as required for a Weyl transformation. Therefore, $\Omega$ ensures that the gauged action is Weyl invariant. In order to restrict the gauge group to the superdiffeomorphisms alone, $\Omega$ has to be set to a fixed value. Thus $\Omega$ is a compensator.

\subsection{Improvement Terms}

From the point of view of linear supergravity, the supersymmetry current and the stress-energy tensor are obtained by variation of the action (5.4) with respect to the gravitino and the metric respectively. We now explain how to obtain the various conserved currents differing by improvement terms. The procedure is analogous to that used in the Weyl gauging [54 to understand the relation between non-minimal coupling and improvement terms for the stress-energy tensor of non-supersymmetric theories. The key point is that the divergence of the gauge potential for the scale symmetry transforms like the Ricci scalar $R$ under diffeomorphisms and Weyl transformations. We start by explaining how this works here at the component level and show how the Weyl gauging is done at the level of the multiplets within the superfield Noether procedure. For simplicity we first consider a generic $\mathcal{N}=1$ theory.

Components We can identify the component in $B$ and $C$ transforming like the Ricci scalar $R$ by noting that

$$
\delta\left(D^{2} \bar{D}^{2} B\right)=-96 i \square \bar{\sigma}, \quad \delta\left(D^{2} \bar{D}^{2} C\right)=96 i \square \bar{\sigma} .
$$

Therefore, the highest component, $d$, of $B$ (and similarly for $C$ ) is a scalar that transforms like $R$, which, at the linear level, is given by $R=\partial_{\mu} \partial_{\nu} g^{\mu \nu}-\square g^{\mu}{ }_{\mu}$. Therefore, the component $d$ can either be considered as independent of the metric or to be $d^{\prime}+b_{1} R$. It is then clear that variation of (5.4) with respect to the metric will give an improvement 
term of the form $b_{1}\left(\eta^{\mu \nu} \square-\partial^{\mu} \partial^{\nu}\right)(X+\bar{X})$ for $T^{\mu \nu}$, which is what we were looking for. We can repeat the same procedure with the second highest component of $B$ (and $C$ ) to improve the supersymmetry current.

Superfield In order to see how this procedure lifts to superspace, we recall the identities (2.13) satisfied by the parameter superfield $h^{\alpha \dot{\alpha}}$. Take now a representative triplet of currents, $(T, X, J)$ say, and the corresponding linearized action (5.4). Now, due to (2.13), the variations of these potentials are not independent. Indeed we have

$$
\begin{aligned}
& \delta B+\frac{1}{12}\left[D_{\alpha}, \bar{D}_{\dot{\alpha}}\right] \delta H^{\alpha \dot{\alpha}}+8 i(\delta \Omega+\delta \bar{\Omega})=0 \\
& \delta C+\frac{3}{2} i \partial_{\alpha \dot{\alpha}} \delta H^{\alpha \dot{\alpha}}+24 i(\delta \Omega-\delta \bar{\Omega})=0 .
\end{aligned}
$$

In particular, if we replace $C$ in (5.4) by $-\frac{3}{2} i \partial_{\alpha \dot{\alpha}} H^{\alpha \dot{\alpha}}-24 i(\Omega-\bar{\Omega})$, then the total action obtained in this way is also invariant. The effect of this substitution is, as in the component approach, to relate the top components of $C$ to the metric and gravitino, but this time in a supersymmetric way.

In order to make the equivalence with adding improvement terms explicit, we take two superfields $U$ and $V$ and rewrite (5.4) as

$$
\begin{aligned}
& \frac{i}{16} \int d^{8} z\left\{H^{\alpha \dot{\alpha}} T_{\alpha \dot{\alpha}}+B(X+\bar{X}+U)+C(X-\bar{X}+V)-B U-C V\right\} \\
& -\frac{1}{2} \int d^{6} z_{+} \Omega J-\frac{1}{2} \int d^{6} z_{-} \bar{\Omega} \bar{J} .
\end{aligned}
$$

Then, using (5.8), we replace (5.9) by

$$
\begin{aligned}
\frac{i}{16} \int d^{8} z & \left\{H^{\alpha \dot{\alpha}} T_{\alpha \dot{\alpha}}+B(X+\bar{X}+U)+C(X-\bar{X}+V)\right. \\
& \left.-\left[-\frac{1}{12}\left[D_{\alpha}, \bar{D}_{\dot{\alpha}}\right] H^{\alpha \dot{\alpha}}-8 i(\Omega+\bar{\Omega})\right] U-\left[-\frac{3 i}{2} \partial_{\alpha \dot{\alpha}} H^{\alpha \dot{\alpha}}-24 i(\Omega-\bar{\Omega})\right] V\right\} \\
& -\frac{1}{2} \int d^{6} z_{+} \Omega J-\frac{1}{2} \int d^{6} z_{-} \bar{\Omega} \bar{J} .
\end{aligned}
$$

The total action obtained in this way is, of course, also invariant under superdiffeomorphisms and Weyl transformations. Integrating by parts and regrouping the terms in $\Omega$ and $\bar{\Omega}$, we end up with

$$
\begin{aligned}
\frac{i}{16} \int d^{8} z\left\{H^{\alpha \dot{\alpha}}\right. & {\left[T_{\alpha \dot{\alpha}}+\frac{1}{12}\left[D_{\alpha}, \bar{D}_{\dot{\alpha}}\right] U-\frac{3 i}{2} \partial_{\alpha \dot{\alpha}} V\right] } \\
+ & B(X+\bar{X}+U)+C(X-\bar{X}+V)\} \\
- & \frac{1}{2} \int d^{6} z_{+} \Omega\left(J+\bar{D}^{2} U+3 \bar{D}^{2} V\right) \\
& -\frac{1}{2} \int d^{6} z_{-} \bar{\Omega}\left(\bar{J}+D^{2} U-3 D^{2} V\right) .
\end{aligned}
$$

Therefore, starting with the coupling (5.4) and the representative $(T, X, J)$, we have constructed a different coupling (5.11) associated with another representative $\left(T^{\prime}, X^{\prime}\right.$, $J^{\prime}$ ). This shows explicitly how the equivalence relation (2.14) relates different supergravities to each other. In particular, if the matter action is conformal, we can obtain the conformal $\mathcal{N}=1$ supergravity [21, 37] in this way. 
Comparison with the literature: Let us now see how we can recover the existing results in the literature [6, 21, 22, 23, 24] on the various linearized $\mathcal{N}=1$ supergravities from our formalism. For this, we first recall the conservation equation (3.6)

$$
\bar{D}^{\dot{\alpha}} T_{\alpha \dot{\alpha}}+W_{\alpha}+D_{\alpha} \tau=0
$$

with

$$
W_{\alpha} \equiv \frac{1}{4} \bar{D}^{2} D_{\alpha}(X-2 \bar{X}), \quad \tau \equiv \frac{1}{4} \bar{D}^{2} X-\frac{1}{6} J .
$$

Let us now furthermore impose the restriction that $X$ is purely imaginary, $X=-\bar{X}$. In this case we can compare the conservation equation (5.12) with eq. (3.4) in [24]. The case $n=-\frac{1}{3}$ (old minimal) in [24] then corresponds to $W_{\alpha}=0$ in (5.12), whereas $n=0$ (new minimal) in 24] corresponds to $\tau=0$. For all other real $n$, eq.(3.4) in [24], with $\lambda_{\beta}=D_{\beta} \Gamma$, can be written as

$$
\bar{D}^{\dot{\alpha}} J_{\alpha \dot{\alpha}}=\frac{i}{6} \bar{D}^{2} D_{\alpha}(\Gamma+\bar{\Gamma})-\frac{i}{3} \frac{1}{3 n+1} D_{\alpha} \bar{D}^{2} \bar{\Gamma} .
$$

This then agrees with (5.12) provided we identify

$$
\begin{aligned}
\frac{i}{6}(\bar{\Gamma}+\Gamma) & =-\frac{3}{2} X \text { and } \\
\frac{i}{3(3 n+1)} \bar{D}^{2} \bar{\Gamma} & =\tau=-\frac{1}{6} J+\frac{1}{2} \bar{D}^{2} X .
\end{aligned}
$$

For a general $W_{\alpha}(X$ and $\bar{X}$ unrelated $)$ and $\tau=0$, the multiplet has $(16+16)$ components. If furthermore $\tau \neq 0$, we count $(20+20)$ components, which agrees with the non minimal supergravity.

Finally, if we impose no relation between the various improvement terms, we showed in subsection 3.1 .3 that we have to count all components of $X$. So, we end up with $(28+28)$ components, which agrees with the number of components of the flexible supergravity proposed in [25].

\section{$5.2 \mathcal{N}=2$ Supergravity}

To obtain a linearized $\mathcal{N}=2$ supergravity we can start with the $\mathcal{N}=2$ vector multiplet discussed is subsection 4.2. In analogy with the $\mathcal{N}=1$ case, we introduce the potentials $\Lambda, \Omega$ and a coupling

$$
\int d^{12} z \Lambda T+\int d^{8} z_{+} \Omega \mathcal{J}+\int d^{8} z_{-} \bar{\Omega} \overline{\mathcal{J}}
$$

The corresponding transformations are

$$
\delta \Lambda \equiv-i(H-\bar{H}), \quad \delta \Omega \equiv 144 i \sigma .
$$

Note that the Weyl multiplet $\Lambda$, which was derived in 53 within harmonic superspace, arises here in ordinary superspace as a direct consequence of the localized transformation (4.8) of the vector multiplet $\mathcal{A}$.

To see how the improvement terms are traced to supergravity couplings we again follow the same path as for $\mathcal{N}=1$. Concentrating on the stress-energy tensor we notice 
that the improvement term is a double derivative of lowest component in the supercurrent $T$. Thus the highest component of $\Lambda$ transforms like the Ricci scalar and therefore we are free to relate it to the metric. What is different here is that there is no relation of the type (5.8) between the variation of the different superpotentials. However, we can nevertheless implement the substitution at the superspace level by expressing $\Lambda$ in terms of the unconstrained spinor superfield $\psi_{\alpha \mathbf{i}}$ of Poincaré supergravity [50] as

$$
\Lambda \equiv D^{\alpha \mathbf{i}} \psi_{\alpha \mathbf{i}}+\bar{D}_{\dot{\alpha} \mathbf{i}} \bar{\psi}^{\dot{\alpha} \mathbf{i}}
$$

This has indeed the effect of relating the highest component of $\Lambda$ to the metric.

\section{Multiplet of Currents}

In this section we present the algebra of the component Noether currents and exhibit the on-shell multiplet structure of a general, i.e. not necessarily minimal, supermultiplet.

\section{1 $\mathcal{N}=1$ Multiplets}

We start from the variation of the energy-momentum tensor, obtained in the same manner as the supersymmetry current (3.44) in section 3 , by choosing $L^{\alpha}=\bar{\theta}_{\dot{\alpha}} a^{\alpha \dot{\alpha}}\left(x_{-}\right)$. We then find

$\delta S=-2 \int d^{4} x a^{\alpha \dot{\alpha}}\left(\partial_{\alpha \dot{\beta}} \bar{D}^{\dot{\beta}} D^{\beta} \widetilde{T}_{\beta \dot{\alpha}}-\partial_{\beta \dot{\alpha}} D^{\beta} \bar{D}^{\dot{\beta}} \widetilde{T}_{\alpha \dot{\beta}}-\partial_{\beta \dot{\beta}} \bar{D}_{\dot{\alpha}} D^{\beta} \widetilde{T}_{\alpha}^{\dot{\beta}}+\partial_{\beta \dot{\beta}} D_{\alpha} \bar{D}^{\dot{\beta}} \widetilde{T}_{\dot{\alpha}}^{\beta}\right) \mid$

from which we extract the symmetric energy-momentum tensor:

$$
\begin{aligned}
T_{\mu \nu}= & \left(4 \sigma_{(\mu}^{\alpha \dot{\alpha}}\left[D_{\alpha}, \bar{D}_{\dot{\alpha}}\right] \widetilde{T}_{\nu)}-4 \eta_{\mu \nu} \sigma^{\rho \alpha \dot{\alpha}}\left[D_{\alpha}, \bar{D}_{\dot{\alpha}}\right] \widetilde{T}_{\rho}\right. \\
& \left.+\left(\partial_{\mu} \partial_{\nu}-\eta_{\mu \nu} \square\right)\left(b_{1}(X+\bar{X})+i b_{2}(X-\bar{X})\right)\right) \mid
\end{aligned}
$$

The two real constants $b_{1}$ and $b_{2}$ introduced here correspond to improvement terms. Its trace is given by:

$$
T_{\mu}^{\mu}=\left(-2\left(D^{2} \widetilde{J}+\bar{D}^{2} \overline{\widetilde{J}}\right)-3 \square\left(b_{1}(X+\bar{X})+i b_{2}(X-\bar{X})\right)\right) \mid
$$

Using (3.44), the supersymmetry transformation of $T_{\mu \nu}$ can then b written as

$$
\begin{aligned}
\delta_{\alpha} T_{\mu \nu}= & 8 \sigma_{\rho(\mu \alpha}^{\beta} \partial^{\rho} j_{\nu) \beta} \\
& +\left(\left(\partial_{\mu} \partial_{\nu}-\eta_{\mu \nu} \square\right) D_{\alpha}\left(\left(b_{1}+2 a_{1}\right)(X+\bar{X})+\left(i b_{2}+2 a_{2}\right)(X-\bar{X})\right)\right) \mid .
\end{aligned}
$$

It contains not only the derivatives of the supersymmetry current, as expected, but also the derivatives of $D_{\alpha} X \mid$, which shows that $D_{\alpha} X \mid$ belongs to the multiplet. The 
variations of the supersymmetry current, in turn, are given by

$$
\begin{aligned}
\delta_{\alpha} j_{\mu \beta}= & \sigma_{\mu \nu \alpha \beta} \partial^{\nu}\left(\frac{8}{3} \overline{\widetilde{J}}+\frac{1}{2} D^{2}\left(a_{1}(X+\bar{X})+a_{2}(X-\bar{X})\right)\right) \mid \\
\sigma_{\mu}^{\alpha \dot{\alpha}} \bar{\delta}_{\dot{\alpha}} j_{\nu \alpha}= & \frac{i}{2} T_{\mu \nu}-4 \partial_{\mu} \widetilde{j}_{\nu}^{(5)}+4 \eta_{\mu \nu} \partial^{\rho} \widetilde{j}_{\rho}^{(5)}-2 i \varepsilon_{\mu \nu}{ }^{\rho \lambda} \partial_{\rho} \widetilde{j}_{\lambda}^{(5)} \\
& +\frac{3 i}{8} \varepsilon_{\mu \nu}{ }^{\rho \lambda} \sigma_{\lambda}^{\alpha \dot{\alpha}}\left(\left[D_{\alpha}, \bar{D}_{\dot{\alpha}}\right] \partial_{\rho}\left(a_{1}(X+\bar{X})+a_{2}(X-\bar{X})\right)\right) \mid
\end{aligned}
$$

where we have defined

$$
\begin{aligned}
\widetilde{j}_{\mu}^{(5)}= & \left(\widetilde{T}_{\mu}+\frac{1}{16} \sigma_{\mu}^{\alpha \dot{\alpha}}\left[D_{\alpha}, \bar{D}_{\dot{\alpha}}\right]\left(a_{1}(X+\bar{X})+a_{2}(X-\bar{X})\right)\right. \\
& \left.+\frac{i}{8} \partial_{\mu}\left(\left(b_{1}+2 a_{1}\right)(X+\bar{X})+\left(i b_{2}+2 a_{2}\right)(X-\bar{X})\right)\right) \mid .
\end{aligned}
$$

Therefore, both $X \mid$ and $\widetilde{J} \mid$ have to be included in the multiplet. In turn, this implies that the multiplet is formed by the components of the superfields $\widetilde{T}, \widetilde{J}$ and $X$.

\section{2 $\mathcal{N}=2$ Vector Multiplet}

With $L_{\mathbf{i j}}=-\frac{1}{18} \theta_{(\mathbf{i}}^{\alpha} \bar{\theta}_{\mathbf{j}}^{3 \dot{\alpha}} a_{\alpha \dot{\alpha}}\left(x^{+}\right)$, one finds

$$
\begin{gathered}
T^{\mu \nu}=-24\left[\frac{1}{2} \eta^{\mu \nu}\left\{D^{\mathbf{i j}}, \bar{D}_{\mathbf{i j}}\right\} T-\frac{3}{2} \bar{\sigma}^{\mu \dot{\alpha} \alpha} \bar{\sigma}^{\nu \dot{\beta} \beta}\left\{D_{\alpha \beta}, \bar{D}_{\dot{\alpha} \dot{\beta}}\right\} T\right. \\
\left.+48 \eta^{\mu \nu} \square T-b\left(\eta^{\mu \nu} \square-\partial^{\mu} \partial^{\nu}\right) T\right] \mid .
\end{gathered}
$$

Again, on-shell the first term in the r.h.s. of $(6.8)$ is equal to $-12 i \eta^{\mu \nu}\left(D^{4} \mathcal{J}-\bar{D}^{4} \overline{\mathcal{J}}\right)$. Next we determine also the value of $b$ for the two interesting cases. The second term in the r.h.s. of $(\sqrt[6.8]{)})$ is traceless and thus we immediately have

$$
T_{\mu}^{\mu}=-48 i\left(D^{4} \mathcal{J}-\bar{D}^{4} \overline{\mathcal{J}}\right)|+72(b-64) \square T| .
$$

Therefore, this trace vanishes again when $\mathcal{J}=0$ and $b=64$.

The value of $b$ corresponding to the canonical stress-energy tensor can only be fixed at the components level. For this purpose, we consider classical Yang-Mills theory. All the fields being decoupled, it is enough to determine the contribution from the scalar field $\phi \equiv \mathcal{A} \mid$ to the Hamiltonian density $T^{00}$. So, suppose that $\mathcal{F}(\mathcal{A}) \propto \mathcal{A}^{2}$. It follows then from (4.14) that $T=\mathcal{A} \overline{\mathcal{A}}$, up to some global factor, and that $\mathcal{J}=0$. Consider then the different terms of (6.8).

- As $\mathcal{J}=0$, the first term vanishes on-shell.

- For the second term, when all the fields except $\phi$ are set to zero, we have:

$$
\left\{D_{\alpha \beta}, \bar{D}_{\dot{\alpha} \dot{\beta}}\right\} T\left|=\mathcal{A} D_{\alpha \beta} \bar{D}_{\dot{\alpha} \dot{\beta}} \overline{\mathcal{A}}\right|+\text { c.c. }=16 \mathcal{A} \partial_{(\alpha(\dot{\alpha}} \partial_{\beta) \dot{\beta})} \overline{\mathcal{A}} \mid+ \text { c.c. }
$$

where we have used the chirality of $\mathcal{A}$ and the relation (D.5) of appendix $\mathrm{D}$. Using the identity $\bar{\sigma}^{\mu \dot{\alpha} \alpha} \bar{\sigma}^{\nu \dot{\beta} \beta} \partial_{(\alpha(\dot{\alpha}} \partial_{\beta) \dot{\beta})}=-\eta^{\mu \nu} \square+4 \partial^{\mu} \partial^{\nu}$, the second term gives a contribution:

$$
24 \phi\left(-\eta^{\mu \nu} \square \bar{\phi}+4 \partial^{\mu} \partial^{\nu} \bar{\phi}\right)+\text { c.c. }=96 \phi \partial^{\mu} \partial^{\nu} \bar{\phi}+\text { c.c. }
$$


where we have used the equation of motion $\square \bar{\phi}=0$.

- The contribution of the last terms is simply: $(b-48) \eta^{\mu \nu} \square(\phi \bar{\phi})-b \partial^{\mu} \partial^{\nu}(\phi \bar{\phi})$. Therefore, on-shell,

$$
T^{\mu \nu} \propto 2(b-48) \eta^{\mu \nu} \partial^{\rho} \phi \partial_{\rho} \bar{\phi}+96 \phi \partial^{\mu} \partial^{\nu} \bar{\phi}+96 \bar{\phi} \partial^{\mu} \partial^{\nu} \phi-b \partial^{\mu} \partial^{\nu}(\phi \bar{\phi})
$$

The Hamiltonian density is then

$$
\begin{aligned}
& T^{00} \propto-(2 b-96)\left[-\left|\partial_{0} \phi\right|^{2}+\left|\partial_{i} \phi\right|^{2}\right]+(96-b) \partial^{0} \partial^{0}(\phi \bar{\phi})-192 \partial^{0} \phi \partial^{0} \bar{\phi} \\
& T^{00} \propto-(2 b-96)\left|\partial_{i} \phi\right|^{2}+(2 b-96-192)\left|\partial_{0} \phi\right|^{2}+(96-b) \partial^{0} \partial^{0}(\phi \bar{\phi})
\end{aligned}
$$

where we have used $\eta^{00} \equiv-1$. Therefore, $T^{00}$ corresponds to the Hamiltonian density if $-(2 b-96)=2 b-96-192$ and $96-b=0$ i.e. when $b=96$.

Note that in full generality, improvement terms proportional to $\mathcal{J}$ could be added to the supersymmetry current and to the stress-energy tensor above. However, we do not consider such a possibility here.

$S U(2)_{R}$ current We obtain

$$
R_{\mathrm{ij}}^{\mu}=-864 i \sigma_{\alpha \dot{\alpha}}^{\mu}\left[D_{(\mathbf{i}}^{\alpha}, \bar{D}_{\mathbf{j})}^{\dot{\alpha}}\right] T \mid
$$

\subsubsection{Supersymmetry Transformations of the Currents}

The next few subsections are devoted to identifying the super multiplets of Noether currents [47, 6, 15, 48] for a given choice of improvement terms. For this, we first give the supersymmetry transformations of the conserved currents. The result is:

$$
\begin{aligned}
\delta_{\alpha \mathbf{i}} R_{\mathbf{j} \mathbf{k}}^{\mu} & =\frac{3}{2} \varepsilon_{\mathbf{i}(\mathbf{j}}\left[J_{\alpha \mathbf{k})}^{\mu}-(24+a) \sigma_{\alpha}^{\mu \nu} \beta \partial_{\nu} D_{\beta \mathbf{k}} T \mid\right] \\
\bar{\delta}_{\dot{\alpha}}^{\mathbf{i}} J_{\alpha}^{\mu \mathbf{j}} & =\varepsilon^{\mathbf{i j}} \sigma_{\nu \alpha \dot{\alpha}}\left[-2 i T^{\mu \nu}+48 i(b-48+2 a)\left(\eta^{\mu \nu} \square-\partial^{\mu} \partial^{\nu}\right) T \mid\right] \\
& +\varepsilon^{\mathbf{i j}}\left\{\frac{1}{4}[a+24]\left[\sigma_{\alpha \dot{\alpha}}^{\mu} \partial^{\rho} \mathcal{R}_{\rho}-\partial_{\alpha \dot{\alpha}} \mathcal{R}^{\mu}\right]-\frac{i a}{4} \varepsilon^{\mu \nu \rho \tau} \sigma_{\tau \alpha \dot{\alpha}} \partial_{\nu} \mathcal{R}_{\rho}\right\} \\
& +\frac{i}{36}(a-24) \partial_{\alpha \dot{\alpha}} R^{\mu \mathbf{i j}}-\frac{1}{36}(a+24) \varepsilon^{\mu \nu \rho \tau} \sigma_{\tau \alpha \dot{\alpha}} \partial_{\nu} R_{\rho}^{\mathbf{i j}}, \\
\delta_{\alpha \mathbf{i}} J_{\beta \mathbf{j}}^{\mu} & =\partial_{\nu}\left\{24 i \varepsilon_{\alpha \beta} \varepsilon_{\mathbf{i j}} Z^{\mu \nu}+96(a+24)\left[\varepsilon_{\mathbf{i j}} \sigma_{(\alpha}^{\mu \nu \gamma} D_{\beta) \gamma} T\left|+\sigma_{\alpha \beta}^{\mu \nu} D_{\mathbf{i j}} T\right|\right]\right\}, \\
\delta_{\alpha \mathbf{i}} T^{\mu \nu} & =\sigma_{\alpha}^{(\mu \rho \beta} \partial_{\rho} J_{\beta \mathbf{i}}^{\nu)}+24(b-48+2 a)\left(\eta^{\mu \nu} \square-\partial^{\mu} \partial^{\nu}\right) D_{\alpha \mathbf{i}} T \mid .
\end{aligned}
$$

where we have introduced

$$
\mathcal{R}^{\mu} \equiv-48 \sigma_{\alpha \dot{\alpha}}^{\mu}\left[D^{\alpha \mathbf{i}}, \bar{D}_{\mathbf{i}}^{\dot{\alpha}}\right] T \mid
$$

It follows from (D.6) that $\partial^{\mu} \mathcal{R}_{\mu}=-8 i\left[D^{\mathrm{ij}}, \bar{D}_{\mathbf{i j}}\right] T$, or, using the equation of conservation (4.15), that

$$
\partial^{\mu} \mathcal{R}_{\mu}=8\left(D^{4} \mathcal{J}+\bar{D}^{4} \overline{\mathcal{J}}\right)
$$




\subsubsection{Multiplet Structure: General Discussion}

Let us now determine with which other components of $T, \mathcal{J}$, and $\overline{\mathcal{J}}$, the above Noether currents form a multiplet. For this we first note that, contrary to the situation in $\mathcal{N}=1$, for a $\mathcal{N}=2$ superconformal theory, the conserved $R$ and $S U(2)_{R}$ and the traceless stress-energy tensor alone can not form a multiplet with the traceless supersymmetry currents. This is so because the number of bosonic components, i.e. $3+9+5=17$ differs from the 16 fermionic components of $J_{\alpha \mathrm{i}}^{\mu}$. Hence the improved multiplet contains other components than the Noether currents above.

The following discussion closely follows that for $N=1$. We start with the variation (6.18) of the stress-energy tensor $T^{\mu \nu}$. Two cases have to be considered.

- $b-48+2 a=0$ : we consider then the variation (6.16) of $J_{\alpha \mathrm{i}}^{\mu}$. As $b-48+2 a=0$, we conclude that $\mathcal{R}^{\mu}$ belongs to the multiplet. However, we then have

$$
\delta_{\alpha \mathbf{i}} \mathcal{R}^{\mu}=16 i\left[\frac{1}{192} J_{\alpha \mathbf{i}}^{\mu}-2 \sigma_{\alpha \dot{\alpha}}^{\mu} \bar{D}_{\mathbf{i}}^{3 \dot{\mathcal{J}}} \overline{\mathcal{J}}\left|-(a+24) \sigma_{\alpha}^{\mu \nu}{ }_{\nu} \partial_{\nu} D_{\beta \mathbf{i}} T\right|\right] .
$$

Using the relation (4.17) for the trace of $J_{\alpha \mathbf{i}}^{\mu}$, we can eliminate $\bar{D}_{\mathbf{i}}^{3 \dot{\mathcal{J}}} \overline{\mathcal{J}}$ to obtain

$$
\delta_{\alpha \mathbf{i}} \mathcal{R}^{\mu}=16 i\left\{\frac{1}{192}\left[\frac{3}{2} J_{\alpha \mathbf{i}}^{\mu}+\sigma_{\alpha}^{\mu \nu \beta} J_{\nu \beta \mathbf{i}}\right]-\frac{3}{4}(8+a) \partial^{\mu} D_{\alpha \mathbf{i}} T\left|+\frac{1}{2}(a-24) \sigma_{\alpha}^{\mu \nu \beta} \partial_{\nu} D_{\beta \mathbf{i}} T\right|\right\} .
$$

This shows that $\chi_{\alpha \mathbf{i}} \equiv D_{\alpha \mathbf{i}} T \mid$ also belongs to the multiplet. We then continue with the variations of $\chi_{\alpha \mathrm{i}}$. Defining $t \equiv T \mid$, and $u_{\alpha \beta} \equiv D_{\alpha \beta} T \mid$, we find

$$
\begin{aligned}
& \delta_{\alpha \mathbf{i}} \chi_{\beta \mathbf{j}}=\frac{1}{2} \varepsilon_{\mathbf{i j}} u_{\alpha \beta}-\frac{i}{2} \varepsilon_{\alpha \beta} \bar{D}_{\mathbf{i j}} \overline{\mathcal{J}} \mid, \\
& \bar{\delta}_{\dot{\alpha} \mathbf{i}} \chi_{\alpha \mathbf{j}}=\frac{i}{3456} R_{\alpha \dot{\alpha} \mathbf{j}}+\frac{1}{384} \varepsilon_{\mathbf{i j}} \mathcal{R}_{\alpha \dot{\alpha}}+i \varepsilon_{\mathbf{i j}} \partial_{\alpha \dot{\alpha}} t .
\end{aligned}
$$

From the first variation (6.23) we conclude that $\bar{D}_{\mathrm{ij}} \overline{\mathcal{J}} \mid$ and therefore all the components of $\overline{\mathcal{J}}$ belong to the multiplet. The second variation (6.24) shows that $t=T \mid$ belongs to the multiplet. As $t$ is the lowest component of $T$, this shows that the multiplet contains $T$. On the other hand, as $T, \mathcal{J}$ and $\overline{\mathcal{J}}$ are the only superfields present, we have shown that, for any value of $a$ and $b$ such that $b-48+2 a=0$, the multiplet of currents corresponds to $T, \mathcal{J}$ and $\overline{\mathcal{J}}$ constrained by the equations of conservation (4.15).

- $b-48+2 a \neq 0$ : in that case, we proceed as follows: the variation (6.18) of $T^{\mu \nu}$ shows that $\chi_{\alpha \mathbf{i}}$ belongs to the multiplet of currents. However, we consider then the variation (6.24) of $\chi_{\alpha \mathbf{i}}$. As $\mathcal{R}^{\mu}$ and $t$ are real, this shows that both of them belong to the multiplet. Therefore, the conclusion is the same as in the preceding case.

Let us now choose a convenient set of independent components of $T, \mathcal{J}$ and $\overline{\mathcal{J}}$. We take $R_{\mathrm{ij}}^{\mu}, J_{\alpha \mathbf{i}}^{\mu}, T^{\mu \nu}, \mathcal{R}^{\mu}, Z^{\mu \nu}, t, \chi_{\alpha \mathbf{i}}, u_{\alpha \beta}, \overline{\mathcal{J}}\left|, \bar{D}_{\dot{\alpha} \mathbf{i}} \overline{\mathcal{J}}\right|$ and $\bar{D}_{\mathbf{i j}} \overline{\mathcal{J}} \mid$. Indeed, first, $\bar{D}_{\dot{\alpha} \dot{\beta}} \overline{\mathcal{J}} \mid$ is the anti self-dual part of $Z^{\mu \nu}$ as given by the equation (4.19). Secondly, $\bar{D}^{3} \overline{\mathcal{J}} \mid$ and $\bar{D}^{4} \overline{\mathcal{J}} \mid$ are related to other components via the 'trace' equations (4.17), (6.9) and (6.20). In order to count the number of components of that multiplet, we need to distinguish between the cases where $\mathcal{J} \neq 0$ and $\mathcal{J}=0$ respectively. 


\subsubsection{Multiplet Structure: Case $\mathcal{J} \neq 0$}

In the general case, the theory is neither $R$-invariant nor conformal invariant. For any value of $a$ and $b$, we have the following number of components.

$$
\begin{aligned}
& \mathcal{R}^{\mu}(4), \quad R_{\mathbf{i j}}^{\mu}(9), \quad J_{\alpha \mathbf{i}}^{\mu}(-24), \quad T^{\mu \nu}(6), \quad Z^{\mu \nu}(6), \\
& t(1), \quad \chi_{\alpha \mathbf{i}}(-8), \quad u_{\alpha \beta}(6), \\
& \overline{\mathcal{J}}\left|(2), \quad \bar{D}_{\dot{\alpha} \mathbf{i}} \overline{\mathcal{J}}\right|(-8), \quad \bar{D}_{\mathrm{ij}} \overline{\mathcal{J}} \mid(6) .
\end{aligned}
$$

This forms a $(40+40)$ multiplet. The algebra satisfied by these components is summarized in subsection B.2 of appendix B.

Let us examine the conditions for $R$-invariance. As $H=\bar{H}$ for superconformal transformations, it follows from the variation (4.13) of the action, and from the specific value (A.7) of $L_{\mathbf{i j}}$ for $U(1)_{R}$ transformations, that the condition is that there exists $r^{\mu}$ such that $-96\left(D^{4} \mathcal{J}+\bar{D}^{4} \overline{\mathcal{J}}\right)=\partial_{\mu} r^{\mu}$. Considering local $U(1)_{R}$ transformations, we find that the conserved $R$-current is in that case $\mathcal{R}^{\mu}+r^{\mu}$. However, as in $\mathcal{N}=1$ and as a consequence of our general discussion above, this is $\mathcal{R}^{\mu}$ itself rather than the $R$-current that is in the multiplet. This is to be expected as the supersymmetry current and stress-energy tensor are not traceless. Therefore, the number of components of the multiplet is again $(40+40)$.

\subsubsection{Conformal Case $\mathcal{J}=0$}

When $\mathcal{J}=0$, the theory is conformal invariant and the equation of conservation is $D^{\mathrm{ij}} T=0$. It is also $R$-invariant and $\mathcal{R}^{\mu}$ is the conserved $R$-current. Let us now discuss how the multiplet structure depends on the improvement terms in the conformal case. We consider first the improved multiplet [6, 15, 48] which corresponds to the values $a=-8$ and $b=64$. It has the components

$$
\begin{array}{rrr}
\mathcal{R}^{\mu}(3), \quad R_{\mathbf{i j}}^{\mu}(9), \quad J_{\alpha \mathbf{i}}^{\mu}(-16), & T^{\mu \nu}(5), & u_{\alpha \beta}(6), \\
& t(1), & \chi_{\alpha \mathbf{i}}(-8) .
\end{array}
$$

Its dimension is $(24+24)$. The corresponding transformations of these components are given in 48, 49 and in subsection B.3 of appendix B.

Let us now turn to the canonical multiplet. This corresponds to $a=-24$ and $b=96$. The difference with the improved multiplet is that here $\chi_{\alpha \mathbf{i}}$ and $J_{\alpha}^{\mu \mathbf{i}}$ are not independent. More precisely, when $\mathcal{J}=0$ and for $a=-24$, equation (4.17) becomes $\left(\bar{\sigma}^{\mu} J_{\mu}\right)^{\dot{\alpha} \mathbf{i}}=4608 \partial^{\dot{\alpha} \alpha} \chi_{\alpha}^{\mathbf{i}}$. Thus, the fermionic components of the canonical multiplet are as follows: There are the 8 components of $\chi_{\alpha \mathbf{i}}$. For $J_{\alpha}^{\mu \mathbf{i}}$ we have only to count its traceless part, i.e. 16 components, as the trace is contained in $\chi_{\alpha \mathbf{i}}$. Hence we recover the 24 fermionic components of $T$.

To summarize, for a conformal theory, the canonical multiplet contains the bosonic components $\mathcal{R}^{\mu}(3), R_{\mathrm{ij}}^{\mu}(9),\left(T^{\mu \nu}, t\right)(6), Z^{\mu \nu}(6)$ and the fermionic components $\left(J_{\alpha}^{\mu \mathbf{i}}, \chi_{\alpha \mathbf{i}}\right)(-24)$. Thus the canonical multiplet is also a $(24+24)$ multiplet.

\section{3 $\mathcal{N}=2$ Tensor Multiplet}

In this section, we identify the contributions from $T^{\mathbf{i j k l}}$ to the various conserved currents. The currents for the tensor multiplet are then simply the sum of the terms given 
respectively in equations (4.16), (6.8), (6.14), (6.19) and of the terms from $T^{\mathrm{ijkl}}$ given explicitly below.

Stress-energy Tensor For global translations, it follows from (A.7) that $H_{\mathrm{ijkl}}=$ $\bar{H}_{\mathrm{ijkl}}=0$. It is immediate to see that this also holds for the local $L_{\mathrm{ij}}$ taken in subsection 6.2. Thus, $T^{\mathrm{ijkl}}$ does not contribute to the stress-energy tensor.

$R$-current Again, a short computation indicates that there is no contribution of $T^{\mathrm{ijk}}$ to the $R$-current.

$S U(2)_{R}$ Invariance and associated Current Contrary to the $\mathcal{N}=2$ superPoincaré and $U(1)_{R}$ transformations, for global $S U(2)_{R}$ transformations, we do have $H=\bar{H}$ but $H_{\mathrm{ijkl}} \neq \bar{H}_{\mathrm{ijkl}}$. More precisely, it follows from A.7) that

$$
H_{\mathbf{i j k l}}-\bar{H}_{\mathbf{i j k l}}=-\frac{3 i}{2} \eta_{\mathbf{m}(\mathbf{i}}\left[\theta_{\mathbf{j}}^{\mathbf{m}_{\mathbf{j}}} \bar{\theta}_{\mathbf{k l})}+\bar{\theta}_{\mathbf{j}}^{\mathbf{m}} \theta_{\mathbf{k l})}\right] .
$$

It is nevertheless still possible to express the corresponding $S U(2)_{R}$ current in terms of $T$ and of $T_{\mathrm{ijkl}}$. To see this, we consider first global transformations and concentrate on the term giving problem i.e. the one proportional to $(H-\bar{H})_{\mathrm{ijkl}}$. Its contribution to the variation of the action under $S U(2)_{R}$ transformations after integration on the Grassman variables is

$$
\frac{27 i}{20} \int d^{4} x \eta_{\mathbf{m i}}\left[D^{\mathbf{m}}{ }_{\mathbf{j}} \bar{D}_{\mathbf{k l}}+\bar{D}_{\mathbf{j}}^{\mathbf{m}} D_{\mathbf{k l}}\right] T^{\mathbf{i j k} \mathbf{l}} \mid
$$

However, we prove in appendix 9 that

$$
\left(D_{\mathbf{j}}^{\left(\mathbf{m}_{\mathbf{k} \mathbf{l}}\right.} \bar{D}_{\mathbf{j}} \bar{D}^{\left(\mathbf{m}_{\mathbf{j}}\right.} D_{\mathbf{k l}}\right) T^{\mathbf{i}) \mathbf{j k} \mathbf{l}}=-8 i \partial_{\alpha \dot{\alpha}}\left[D_{\mathbf{k}}^{\alpha}, \bar{D}_{\mathbf{l}}^{\dot{\alpha}}\right] T^{\mathbf{m i k l}}
$$

Thus, as $\eta_{\mathbf{i j}}$ is symmetric, this proves the invariance of the action and gives the contribution of $T^{\mathrm{ijkl}}$ to the $S U(2)_{R}$ current $R_{\mathrm{ij}}^{\mu}$ :

$$
1728 i \sigma_{\alpha \dot{\alpha}}^{\mu}\left[D^{\alpha \mathbf{k}}, \bar{D}^{\dot{\alpha} \mathbf{l}}\right] T_{\mathrm{ijk} \mathbf{l}} \text {. }
$$

\section{Conclusions}

In this paper, we developed a method to construct the various multiplets of Noether currents directly at the superfield level (Superfield Noether Procedure). This formalism is useful in view of a unified treatment of those supersymmetric theories for which an off-shell superfield formulation exists. In particular, it produces a manifestly supersymmetric treatment of the various improvement terms interpolating between canonical and improved Noether currents.

A prominent feature of this formulation is that the various algebraic manipulations are independent of the complexity of the action for a given supermultiplet. This makes this approach particularly suited for dealing with (quantum) effective actions of supersymmetric theories. As a specific application we obtained an efficient algorithm to compute the supersymmetry central charge for an arbitrary local action of a given 
off-shell superfield. As another application we gave a systematic derivation of the supercurrent of the $\mathcal{N}=2$ tensor multiplet as well as the multiplet of canonical Noether currents of $\mathcal{N}=2$ Yang-Mills theory. As a by-product we then also found a simple derivation of the anomalous superconformal Ward-Identity for the effective action of that theory.

Of course, by its very nature the application of our procedure is limited to those theories for which an off-shell superfield formulation exists. This is an obvious limitation when dealing with models with extended supersymmetry. In view of this, it would be interesting to generalize our formalism to harmonic superspace [55].

\section{Acknowledgments}

We acknowledge helpful discussions with F. Delduc, P. Howe, E. Ivanov, S. Kuzenko and E. Sokatchev. This work has been supported by the TMR contract FMRX-CT96-0012 of the European Union, the ACI 2078-CDR-2 program of Ministère de la Recherche and by the DFG-Stringtheorie Schwerpunktsprogramm SPP 1096.

\section{A Superconformal Transformations}

Below we give the explicit form of the parameter superfields for $\mathcal{N}=1$ and $\mathcal{N}=2$ superconformal transformations as well as some useful identities.

\section{A.1 $\mathcal{N}=1$}

The general solution of equations (2.1) and (2.5) is [36]:

$$
\begin{aligned}
h^{\alpha \dot{\alpha}}= & a^{\alpha \dot{\alpha}}+4 i \varepsilon_{\mathbf{i}}^{\alpha} \bar{\theta}^{\dot{\alpha} \mathbf{i}}+4 i \dot{\varepsilon}^{\dot{\alpha} \mathbf{i}} \theta_{\mathbf{i}}^{\alpha}-\omega^{\alpha}{ }_{\beta} x_{-}^{\beta \dot{\alpha}}+\bar{\omega}^{\dot{\alpha}}{ }_{\dot{\beta}} x_{+}^{\alpha \dot{\beta}}-4 \eta \theta_{\mathbf{i}}^{\alpha} \bar{\theta}^{\dot{\alpha} \mathbf{i}}-6 i \eta_{\mathbf{j}}^{\mathbf{i}} \theta_{\mathbf{i}}^{\alpha} \bar{\theta}^{\dot{\alpha} \mathbf{j}} \\
& +\kappa x^{\alpha \dot{\alpha}}+x_{-}^{\beta \dot{\alpha}} b_{\beta \dot{\beta}} x_{+}^{\alpha \dot{\beta}}-x_{-}^{\beta \dot{\alpha}} \rho_{\beta}^{\mathbf{i}} \theta_{\mathbf{i}}^{\alpha}+\bar{\theta}^{\dot{\alpha} \mathbf{i}} \bar{\rho}_{\dot{\beta} \mathbf{i}} x_{+}^{\alpha \dot{\beta}} .
\end{aligned}
$$

The different parameters correspond to translations $a^{\alpha \dot{\alpha}}$, supersymmetry transformations $\varepsilon_{\mathbf{i}}^{\alpha}$, Lorentz transformations $\omega^{\alpha}{ }_{\beta}$ (with $\omega_{\alpha \beta}=\omega_{\beta \alpha}$ and $\left.\omega^{\alpha}{ }_{\alpha}=0\right), U(1)_{R^{-}}$ transformations $\eta$, SU $(\mathcal{N})$-transformations $\eta_{\mathbf{j}}^{\mathbf{i}}\left(\right.$ with $\left(\eta_{\mathbf{j}}^{\mathbf{i}}\right)^{*}=-\eta_{\mathbf{i}}^{\mathbf{j}}$ and $\left.\eta_{\mathbf{i}}^{\mathbf{i}}=0\right)$, dilations $\kappa$, special conformal transformations $b_{\alpha \dot{\alpha}}$ and special superconformal transformations $\rho_{\alpha}^{\mathbf{i}}$. Correspondingly we have

$$
\begin{aligned}
L^{\alpha}= & -\frac{1}{2} a^{\alpha \dot{\alpha}} \bar{\theta}_{\dot{\alpha}}+i \varepsilon^{\alpha} \bar{\theta}^{2}-2 i \bar{\varepsilon}_{\dot{\alpha}} \bar{\theta}^{\dot{\alpha}} \theta^{\alpha}-\frac{1}{2} \omega^{\alpha}{ }_{\beta} x^{\beta \dot{\alpha}} \bar{\theta}_{\dot{\alpha}}+\frac{1}{2} \bar{\omega}^{\dot{\alpha}}{ }_{\beta} x_{+}^{\alpha \dot{\beta}} \bar{\theta}_{\dot{\alpha}}-\eta \theta^{\alpha} \bar{\theta}^{2} \\
& +\frac{1}{2} \kappa x^{\alpha \dot{\alpha}} \bar{\theta}_{\dot{\alpha}}-\frac{i}{2} \kappa \theta^{\alpha} \bar{\theta}^{2}+\frac{1}{2} x^{\beta \dot{\alpha}} b_{\beta \dot{\beta}} x_{+}^{\alpha \dot{\beta}} \bar{\theta}_{\dot{\alpha}}+\frac{1}{2} x^{\beta \dot{\alpha}} \rho_{\beta} \theta^{\alpha} \bar{\theta}_{\dot{\alpha}}-\frac{1}{4} \bar{\theta}^{2} \bar{\rho}_{\dot{\alpha}} x_{+}^{\alpha \dot{\alpha}}
\end{aligned}
$$

The algebra between $\mathcal{L}$ given by (2.9) and $D_{\alpha}^{\mathbf{i}}$ is given by

$$
\left[D_{\alpha}^{\mathbf{i}}, \mathcal{L}\right]=\frac{3}{\mathcal{N}(4-\mathcal{N})}((\mathcal{N}-2) \sigma+2 \bar{\sigma}) D_{\alpha}^{\mathbf{i}}-i \Lambda_{\mathbf{j}}^{\mathbf{i}} D_{\alpha}^{\mathbf{j}}-\Omega_{\alpha}{ }^{\beta} D_{\beta}^{\mathbf{i}}
$$


where $\sigma, \Lambda_{\mathbf{j}}^{\mathbf{i}}$ and $\Omega_{\alpha}{ }^{\beta}$ are defined by

$$
\begin{aligned}
\sigma & =\frac{1}{6}\left(D^{\alpha \mathbf{i}} \lambda_{\alpha \mathbf{i}}-\frac{1}{2} \partial_{\alpha \dot{\alpha}} h^{\alpha \dot{\alpha}}\right) \\
\Lambda_{\mathbf{j}}^{\mathbf{i}} & =-\frac{i}{4}\left(D_{\alpha}^{\mathbf{i}} \lambda_{\mathbf{j}}^{\alpha}+\bar{D}_{\dot{\alpha} \mathbf{j}} \bar{\lambda}^{\dot{\alpha} \mathbf{i}}-\frac{1}{\mathcal{N}} \delta_{\mathbf{j}}^{\mathbf{i}}\left(D_{\alpha}^{\mathbf{k}} \lambda_{\mathbf{k}}^{\alpha}+\bar{D}_{\dot{\alpha} \mathbf{k}} \bar{\lambda}^{\dot{\alpha} \mathbf{k}}\right)\right) \\
\Omega_{\alpha \beta} & =\frac{1}{2-\mathcal{N}}\left(D_{(\alpha}^{\mathbf{i}} \lambda_{\beta) \mathbf{i}}+\frac{1}{2} \partial_{(\alpha \dot{\alpha}} h_{\beta)} \dot{\alpha}\right) .
\end{aligned}
$$

Both $\sigma$ and $\Omega_{\alpha}{ }^{\beta}$ are chiral and $\Lambda^{\mathbf{i}}{ }_{\mathbf{j}}$ is hermitian and traceless, i.e.

$$
\bar{D}_{\dot{\alpha} \mathbf{i}} \sigma=0, \quad \bar{D}_{\dot{\gamma} \mathbf{i}} \Omega_{\alpha}^{\beta}=0, \quad\left(\Lambda_{\mathbf{j}}^{\mathbf{i}}\right)^{*}=\Lambda_{\mathbf{i}}^{\mathbf{j}}, \quad \Lambda_{\mathbf{i}}^{\mathbf{i}}=0
$$

From (2.3) and (A.4), we then obtain

$$
\lambda^{\alpha}=-\frac{i}{4} \bar{D}^{2} L^{\alpha}, \quad \Omega_{\alpha \beta}=\frac{i}{8} \bar{D}^{2} D_{(\alpha} L_{\beta)}, \quad \sigma=-\frac{i}{24} \bar{D}^{2} D^{\alpha} L_{\alpha} .
$$

\section{A.2 $\mathcal{N}=2$}

The interested reader can verify that all global transformations are correctly parametrized by

$$
\begin{aligned}
L_{\mathbf{i j}}= & -\frac{1}{18} \theta_{(\mathbf{i}}^{\alpha} \bar{\theta}_{\mathbf{j})}^{3 \dot{\alpha}}\left[a_{\alpha \dot{\alpha}}-\omega_{\alpha \beta} x_{-\dot{\alpha}}^{\beta}+\bar{\omega}_{\dot{\alpha} \dot{\beta}} x_{+\alpha}^{\dot{\beta}}+\kappa x_{\alpha \dot{\alpha}}\right] \\
& \left.-\frac{i}{9} \varepsilon_{(\mathbf{i}}^{\alpha} \theta_{\alpha \mathbf{j})} \bar{\theta}^{4}+\frac{2 i}{9} \bar{\varepsilon}_{\mathbf{k}}^{\dot{\alpha}} \theta_{\mathbf{i j}} \bar{\theta}_{\dot{\alpha}}^{3 \mathbf{k}}+\frac{1}{18} \eta \theta_{\mathbf{i j}} \bar{\theta}^{4}+\frac{i}{8} \eta_{(\mathbf{i k}} \theta^{\mathbf{k}} \mathbf{j}\right) \bar{\theta}^{4} \\
& -\frac{1}{18} \theta_{(\mathbf{i}}^{\alpha} \bar{\theta}_{\mathbf{j})}^{3 \dot{\alpha}} x_{-\dot{\alpha} \beta} b^{\beta \dot{\beta}} x_{+\dot{\beta} \alpha}-\frac{i}{36} \theta_{\mathbf{i j}} \bar{\theta}^{4} x_{+\alpha \dot{\alpha}} b^{\alpha \dot{\alpha}} \\
& -\frac{5}{144} \bar{\theta}_{\dot{\alpha}(\mathbf{i}}^{3} \theta_{\mathbf{j}) \mathbf{k}} x_{-}^{\dot{\alpha} \beta} \rho_{\beta}^{\mathbf{k}}+\frac{1}{144} \bar{\theta}_{\dot{\alpha} \mathbf{k}}^{3} \theta_{(\mathbf{i}} \mathbf{k}^{\dot{\alpha} \beta} x_{-\mathbf{j})}-\frac{1}{36} \theta_{(\mathbf{i}}^{\alpha} \bar{\theta}^{4} \bar{\rho}_{\dot{\alpha} \mathbf{j})} x_{+\alpha}^{\dot{\alpha}} .
\end{aligned}
$$

For a global superconformal transformation we then have

$$
\begin{aligned}
H=\bar{H} & =-\frac{1}{2} \theta^{\alpha \mathbf{i}} \bar{\theta}_{\mathbf{i}}^{\dot{\alpha}}\left[a_{\alpha \dot{\alpha}}-\omega_{\alpha \beta} x_{-\dot{\alpha}}^{\beta}+\bar{\omega}_{\dot{\alpha} \dot{\beta}} x_{+\alpha}^{\dot{\beta}}+\kappa x_{\alpha \dot{\alpha}}\right] \\
& +\frac{4 i}{3} \varepsilon_{\mathbf{i}}^{\alpha} \theta_{\alpha \mathbf{j}} \bar{\theta}^{\mathbf{i j}}+\frac{4 i}{3} \bar{\varepsilon}_{\mathbf{i}}^{\dot{\alpha}} \bar{\theta}_{\dot{\alpha} \mathbf{j}} \theta^{\mathbf{i} \mathbf{j}}-\frac{2}{3} \eta \theta_{\mathbf{i j}} \bar{\theta}^{\mathbf{i j}}-\frac{3 i}{2} \eta_{\mathbf{i k}} \theta_{\mathbf{j}}^{\mathbf{k}} \bar{\theta}^{\mathbf{i} \mathbf{j}} \\
& -\frac{1}{2} \theta^{\alpha \mathbf{i}} \bar{\theta}_{\dot{\mathbf{i}}}^{\dot{\alpha}} x_{-\dot{\alpha} \beta} b^{\beta \dot{\beta}} x_{+\dot{\beta} \alpha}+\frac{1}{3} \bar{\theta}_{\dot{\alpha} \mathbf{i}} \theta^{\mathbf{i} \mathbf{j}} \rho_{\alpha \mathbf{j}} x_{-}^{\alpha \dot{\alpha}}+\frac{1}{3} \theta_{\dot{\mathbf{i}}}^{\alpha} \bar{\theta}^{\mathbf{i} \mathbf{j}} \bar{\rho}_{\dot{\alpha} \mathbf{j}} x_{+\alpha}^{\dot{\alpha}}
\end{aligned}
$$

\section{B Multiplet of Currents for the $\mathcal{N}=2$ Vector}

In this appendix, we first give some details how to obtain the variation (4.18) of the supersymmetry current for the $N=2$ vector multiplet. Then, we summarize the multiplet structure in the general case and in the improved case.

\section{B.1 Variation of the Supersymmetry Current}

We are interested in the computation of $D_{\alpha \mathbf{i}} J_{\beta \mathbf{j}}^{\mu}$ where $J_{\alpha \mathbf{i}}^{\mu}$ is given by eq.(4.16) i.e.

$$
J_{\alpha}^{\mu \mathbf{i}}=192\left[i \sigma_{\alpha \dot{\alpha}}^{\mu} \bar{D}_{\mathbf{j}}^{\dot{\alpha}} D^{\mathbf{i} \mathbf{j}} T-3 i \bar{\sigma}^{\mu \dot{\alpha} \beta} \bar{D}_{\dot{\alpha}}^{\mathbf{i}} D_{\alpha \beta} T-12 \partial^{\mu} D_{\alpha}^{\mathbf{i}} T+a \sigma_{\alpha}^{\mu \nu \beta} \partial_{\nu} D_{\beta}^{\mathbf{i}} T\right] .
$$


The method consists of course of decomposing all the terms into the symmetric and antisymmetric parts with respect to the $S U(2)_{R}$ and spinor indices.

For the first term in the r.h.s. of $(\overline{B .1})$, we use its equivalent form in terms of $\overline{\mathcal{J}}$ and the chirality of $\overline{\mathcal{J}}$ to get:

$$
\begin{aligned}
D_{\alpha \mathbf{i}}\left[i \sigma_{\beta \dot{\alpha}}^{\mu} \bar{D}_{\mathbf{k}}^{\dot{\alpha}} D_{\mathbf{j}}{ }^{\mathbf{k}} T\right] & =3 i \varepsilon_{\mathbf{i j}} \varepsilon_{\alpha \beta} \bar{\sigma}_{\dot{\alpha} \dot{\beta}}^{\mu \nu} \partial_{\nu} \bar{D}^{\dot{\alpha} \dot{\beta}} \overline{\mathcal{J}}-3 i \varepsilon_{\mathbf{i j}} \sigma_{(\alpha \dot{\alpha}}^{\mu} \sigma_{\beta) \dot{\beta}}^{\nu} \partial_{\nu} \bar{D}^{\dot{\alpha} \dot{\beta}} \overline{\mathcal{J}} \\
& +3 i \varepsilon_{\alpha \beta} \partial^{\mu} \bar{D}_{\mathbf{i j}} \overline{\mathcal{J}}-6 i \sigma_{\alpha \beta}^{\mu \nu} \partial_{\nu} \bar{D}_{\mathbf{i j}} \overline{\mathcal{J}}
\end{aligned}
$$

For the second term in the r.h.s. of $(\mathbb{B . 1})$, we use the relation

$$
\begin{aligned}
D_{\alpha \mathbf{i}} \bar{D}_{\dot{\alpha} \mathbf{j}} D_{\beta \gamma} T & =-2 i \varepsilon_{\mathbf{i j}} \partial_{\alpha \dot{\alpha}} D_{\beta \gamma} T+\frac{2}{3} \varepsilon_{\alpha(\beta} \bar{D}_{\dot{\alpha} \mathbf{j}} D_{\gamma) \mathbf{i}}^{3} T \\
& =-2 i \varepsilon_{\mathbf{i j}} \partial_{\alpha \dot{\alpha}} D_{\beta \gamma} T-2 \varepsilon_{\mathbf{i j}} \varepsilon_{\alpha(\beta} \partial_{\gamma)}^{\dot{\beta}} \bar{D}_{\dot{\alpha} \dot{\beta}} \overline{\mathcal{J}}-2 \varepsilon_{\alpha(\beta} \partial_{\gamma) \dot{\alpha}} \bar{D}_{\mathbf{i j}} \overline{\mathcal{J}}
\end{aligned}
$$

where we have used the equation of conservation (4.15). This enables to get:

$$
\begin{aligned}
D_{\alpha \mathbf{i}}\left[-3 i \bar{\sigma}^{\mu \dot{\alpha} \gamma} \bar{D}_{\dot{\alpha} \mathbf{j}} D_{\beta \gamma} T\right] & =\varepsilon_{\mathbf{i j}} \varepsilon_{\alpha \beta}\left[6 \sigma_{\gamma \delta}^{\mu \nu} D^{\gamma \delta} T+9 i \bar{\sigma}_{\dot{\alpha} \dot{\beta}}^{\mu \nu} \bar{D}^{\dot{\alpha} \dot{\beta}} \overline{\mathcal{J}}\right] \\
& +\varepsilon_{\mathbf{i j}}\left[6 \partial^{\mu} D_{\alpha \beta} T+12 \sigma_{(\alpha}^{\mu \nu} \gamma_{\nu} \partial_{\beta) \gamma} T+3 i \sigma_{(\alpha \dot{\alpha}}^{\mu} \sigma_{\beta) \dot{\beta}}^{\nu} \partial_{\nu} \bar{D}^{\dot{\alpha} \dot{\beta}} \overline{\mathcal{J}}\right] \\
& -9 i \varepsilon_{\alpha \beta} \partial^{\mu} \bar{D}_{\mathbf{i j}} \overline{\mathcal{J}}-6 i \sigma_{\alpha \beta}^{\mu \nu} \partial_{\nu} \bar{D}_{\mathbf{i j}} \overline{\mathcal{J}}
\end{aligned}
$$

For the third and the last terms in the r.h.s. of $(B .1)$ we immediately get:

$$
\begin{aligned}
D_{\alpha \mathbf{i}}\left[-12 \partial^{\mu} D_{\beta \mathbf{j}} T\right] & =-6 \varepsilon_{\mathbf{i j}} \partial^{\mu} D_{\alpha \beta} T-6 \varepsilon_{\alpha \beta} \partial^{\mu} D_{\mathbf{i j}} T \\
& =-6 \varepsilon_{\mathbf{i j}} \partial^{\mu} D_{\alpha \beta} T+6 i \varepsilon_{\alpha \beta} \partial^{\mu} \bar{D}_{\mathbf{i j}} \overline{\mathcal{J}} \\
D_{\alpha \mathbf{i}}\left[a \sigma_{\beta}^{\mu \nu \gamma} \partial_{\nu} D_{\gamma \mathbf{j}} T\right] & =\frac{a}{2} \partial_{\nu}\left[-\frac{1}{2} \varepsilon_{\mathbf{i j}} \varepsilon_{\alpha \beta} \sigma_{\gamma \delta}^{\mu \nu} D^{\gamma \delta} T+\varepsilon_{\mathbf{i j}} \sigma_{(\alpha}^{\mu \nu}{ }^{\gamma} D_{\beta) \gamma} T+\sigma_{\alpha \beta}^{\mu \nu} D_{\mathbf{i j}} T\right]
\end{aligned}
$$

Finally, taking the sum of $(\mathrm{B} .2)$, (B.5), (B.7) and of (B.8) leads to the result (4.18).

\section{B.2 Multiplet in the General Case}

The multiplet is formed of

$$
\begin{aligned}
t= & T \mid, \\
\chi_{\alpha \mathbf{i}}= & D_{\alpha \mathbf{i}} T \mid, \\
u_{\alpha \beta}= & D_{\alpha \beta} T \mid, \\
\mathcal{R}^{\mu}= & -48 \sigma_{\alpha \dot{\alpha}}^{\mu}\left[D^{\alpha \mathbf{i}}, \bar{D}_{\mathbf{i}}^{\dot{\alpha}}\right] T \mid, \\
R_{\mathbf{i j}}^{\mu}= & -864 i \sigma_{\alpha \dot{\alpha}}^{\mu}\left[D_{(\mathbf{i}}^{\alpha}, \bar{D}_{\mathbf{j})}^{\dot{\alpha}}\right] T \mid, \\
J_{\alpha}^{\mu \mathbf{i}}= & 192\left[i \sigma_{\alpha \dot{\alpha}}^{\mu} \bar{D}_{\mathbf{j}}^{\dot{\alpha}} D^{\mathbf{i j}} T\left|-3 i \bar{\sigma}^{\mu \dot{\alpha} \beta} \bar{D}_{\dot{\alpha}}^{\mathbf{i}} D_{\alpha \beta} T\right|-12 \partial^{\mu} \chi_{\alpha}^{\mathbf{i}}+a \sigma_{\alpha}^{\mu \nu \beta} \partial_{\nu} \chi_{\beta}^{\mathbf{i}}\right], \\
T^{\mu \nu}= & -24\left[\frac{1}{2} \eta^{\mu \nu}\left\{D^{\mathbf{i} \mathbf{j}}, \bar{D}_{\mathbf{i j}}\right\} T\left|-\frac{3}{2} \bar{\sigma}^{\mu \dot{\alpha} \alpha} \bar{\sigma}^{\nu \dot{\beta} \beta}\left\{D_{\alpha \beta}, \bar{D}_{\dot{\alpha} \dot{\beta}}\right\} T\right|\right. \\
& \left.+48 \eta^{\mu \nu} \square t-b\left(\eta^{\mu \nu} \square-\partial^{\mu} \partial^{\nu}\right) t\right], \\
Z^{\mu \nu}= & 96\left[\bar{\sigma}_{\dot{\alpha} \dot{\beta}}^{\mu \nu} \bar{D}^{\dot{\alpha} \dot{\beta}} \overline{\mathcal{J}} \mid-i\left(\frac{1}{2}-\frac{a}{48}\right) \sigma_{\alpha \beta}^{\mu \nu} u^{\alpha \beta}\right],
\end{aligned}
$$




$$
\begin{array}{r}
\overline{\mathcal{J}} \mid \\
\bar{D}_{\dot{\alpha i} \mathbf{i}} \overline{\mathcal{J}} \mid \\
\bar{D}_{\mathbf{i j}} \mathcal{J} \mid .
\end{array}
$$

The transformations of these components are

$$
\begin{aligned}
& \delta_{\alpha \mathbf{i}} t=\chi_{\alpha \mathbf{i}}, \\
& \delta_{\alpha \mathbf{i}} \chi_{\beta \mathbf{j}}=\frac{1}{2} \varepsilon_{\mathbf{i j}} u_{\alpha \beta}-\frac{i}{2} \varepsilon_{\alpha \beta} \bar{D}_{\mathbf{i j}} \overline{\mathcal{J}}, \\
& \bar{\delta}_{\dot{\alpha} \mathbf{i}} \chi_{\alpha \mathbf{j}}=\frac{i}{3456} R_{\alpha \dot{\alpha} \mathbf{i j}}+\frac{1}{384} \varepsilon_{\mathbf{i j}} \mathcal{R}_{\alpha \dot{\alpha}}+i \varepsilon_{\mathbf{i j}} \partial_{\alpha \dot{\alpha}} t \\
& \delta_{\alpha \mathbf{i}} u_{\beta \gamma}=4 \varepsilon_{\alpha(\beta} \partial_{\gamma) \dot{\alpha}} \bar{D}_{\mathbf{i}}^{\dot{\alpha}} \overline{\mathcal{J}}, \\
& \bar{\delta}_{\dot{\alpha} \mathbf{i}} u_{\alpha \beta}=\frac{-i}{1152} \sigma_{\mu(\alpha \dot{\alpha}} J_{\beta) \mathbf{i}}^{\mu}+\frac{i}{12}(a-24) \partial_{(\alpha \dot{\alpha}} \chi_{\beta) \mathbf{i}} \text {, } \\
& \delta_{\alpha \mathbf{i}} R_{\mathbf{j k}}^{\mu}=\frac{3}{2} \varepsilon_{\mathbf{i}(\mathbf{j}}\left[J_{\alpha \mathbf{k})}^{\mu}-(24+a) \sigma_{\alpha}^{\mu \nu \beta} \partial_{\nu} \chi_{\beta \mathbf{k})}\right], \\
& \delta_{\alpha \mathbf{i}} \mathcal{R}^{\mu}=16 i\left\{\frac{1}{192}\left[\frac{3}{2} J_{\alpha \mathbf{i}}^{\mu}+\sigma_{\alpha}^{\mu \nu \beta} J_{\nu \beta \mathbf{i}}\right]-\frac{3}{4}(8+a) \partial^{\mu} \chi_{\alpha \mathbf{i}}+\frac{1}{2}(a-24) \sigma_{\alpha}^{\mu \nu \beta} \partial_{\nu} \chi_{\beta \mathbf{i}}\right\}, \\
& \bar{\delta}_{\dot{\alpha}}^{\mathbf{i}} J_{\alpha}^{\mu \mathbf{j}}=\varepsilon^{\mathbf{i j}} \sigma_{\nu \alpha \dot{\alpha}}\left[-2 i T^{\mu \nu}+48 i(b-48+2 a)\left(\eta^{\mu \nu} \square-\partial^{\mu} \partial^{\nu}\right) t\right] \\
& +\varepsilon^{\mathrm{ij}}\left\{\frac{1}{4}[a+24]\left[\sigma_{\alpha \dot{\alpha}}^{\mu} \partial^{\rho} \mathcal{R}_{\rho}-\partial_{\alpha \dot{\alpha}} \mathcal{R}^{\mu}\right]-\frac{i a}{4} \varepsilon^{\mu \nu \rho \tau} \sigma_{\tau \alpha \dot{\alpha}} \partial_{\nu} \mathcal{R}_{\rho}\right\} \\
& +\frac{i}{36}(a-24) \partial_{\alpha \dot{\alpha}} R^{\mu \mathbf{i j}}-\frac{1}{36}(a+24) \varepsilon^{\mu \nu \rho \tau} \sigma_{\tau \alpha \dot{\alpha}} \partial_{\nu} R_{\rho}^{\mathbf{i j}}, \\
& \delta_{\alpha \mathbf{i}} J_{\beta \mathbf{j}}^{\mu}=\partial_{\nu}\left\{24 i \varepsilon_{\alpha \beta} \varepsilon_{\mathbf{i j}} Z^{\mu \nu}+96(a+24)\left[\varepsilon_{\mathbf{i j}} \sigma_{(\alpha}^{\mu \nu \gamma} \chi_{\beta) \gamma}-i \sigma_{\alpha \beta}^{\mu \nu} \bar{D}_{\mathbf{i j}} \overline{\mathcal{J}}\right]\right\}, \\
& \delta_{\alpha \mathbf{i}} T^{\mu \nu}=\sigma_{\alpha}^{(\mu \rho \beta} \partial_{\rho} J_{\beta \mathbf{i}}^{\nu}+24(b-48+2 a)\left(\eta^{\mu \nu} \square-\partial^{\mu} \partial^{\nu}\right) \chi_{\alpha \mathbf{i}}, \\
& \delta_{\alpha \mathbf{i}} Z^{\mu \nu}=-96 i\left(1+\frac{a}{24}\right)\left(\sigma_{\alpha \dot{\alpha}}^{\mu} \partial^{\nu}-\sigma_{\alpha \dot{\alpha}}^{\nu} \partial^{\mu}\right) \bar{D}_{\mathbf{i}}^{\dot{\alpha}} \overline{\mathcal{J}}+96\left(3-\frac{a}{24}\right) \varepsilon^{\mu \nu \rho \tau} \sigma_{\tau \alpha \dot{\alpha}} \partial_{\rho} \bar{D}_{\mathbf{i}}^{\dot{\alpha}} \overline{\mathcal{J}}, \\
& \bar{\delta}_{\dot{\alpha} \mathbf{i}} Z^{\mu \nu}=\left(\eta^{\rho \mu} \sigma_{\alpha \dot{\alpha}}^{\nu}-\eta^{\rho \nu} \sigma_{\alpha \dot{\alpha}}^{\mu}\right)\left(\frac{1}{16} J_{\rho \mathbf{i}}^{\alpha}+\left[\frac{1}{12}(a-24)^{2}+12(8+a)\right] \partial_{\rho} \chi_{\dot{\mathbf{i}}}^{\alpha}\right) \\
& +i \varepsilon^{\mu \nu \rho \tau} \sigma_{\tau \alpha \dot{\alpha}}\left(-\frac{1}{1152}(a+24) J_{\rho \mathbf{i}}^{\alpha}+\left[\frac{1}{12}(a-24)^{2}-12(8+a)\right] \partial_{\rho} \chi_{\dot{\mathbf{i}}}^{\alpha}\right), \\
& \delta_{\alpha \mathbf{i}} \overline{\mathcal{J}}=0 \\
& \bar{\delta}_{\dot{\alpha} \mathbf{i}} \overline{\mathcal{J}}=\bar{D}_{\dot{\alpha} \mathbf{i}} \overline{\mathcal{J}}, \\
& \delta_{\alpha \mathbf{i}}\left(\bar{D}_{\dot{\alpha} \mathbf{j}} \overline{\mathcal{J}}\right)=-2 i \varepsilon_{\mathbf{i j}} \partial_{\alpha \dot{\alpha}} \overline{\mathcal{J}}, \\
& \bar{\delta}_{\dot{\alpha} \mathbf{i}}\left(\bar{D}_{\dot{\beta} \mathbf{j}} \overline{\mathcal{J}}\right)=-\frac{1}{2} \varepsilon_{\dot{\alpha} \dot{\beta}} \bar{D}_{\mathbf{i j}} \overline{\mathcal{J}}+\varepsilon_{\mathbf{i j}} \frac{1}{384} \bar{\sigma}_{\dot{\alpha} \dot{\beta}}^{\mu \nu} Z_{\mu \nu}, \\
& \delta_{\alpha \mathbf{i}}\left(\bar{D}_{\mathbf{j k}} \overline{\mathcal{J}}\right)=-4 i \varepsilon_{\mathbf{i}(\mathbf{j}} \partial_{\alpha \dot{\alpha}} \bar{D}_{\mathbf{k})}^{\dot{\alpha}} \overline{\mathcal{J}} \\
& \bar{\delta}_{\dot{\alpha} \mathbf{i}}\left(\bar{D}_{\mathbf{j k}} \overline{\mathcal{J}}\right)=\frac{1}{6} \varepsilon_{\mathbf{i}(\mathbf{j}}\left[\frac{1}{192}\left(\bar{\sigma}^{\mu} J_{\mu \mathbf{k})}\right)_{\dot{\alpha}}-\frac{3}{2}(8+a) \partial_{\alpha \dot{\alpha}} \chi_{\mathbf{k})}^{\alpha}\right] .
\end{aligned}
$$

\section{B.3 Improved Multiplet}

The multiplet of improved currents is composed of:

$$
\begin{aligned}
t & =T \mid, \\
\chi_{\alpha \mathbf{i}} & =D_{\alpha \mathbf{i}} T \mid,
\end{aligned}
$$




$$
\begin{aligned}
u_{\alpha \beta} & =D_{\alpha \beta} T \mid \\
\mathcal{R}^{\mu} & =-48 \sigma_{\alpha \dot{\alpha}}^{\mu}\left[D^{\alpha \mathbf{i}}, \bar{D}_{\mathbf{i}}^{\dot{\alpha}}\right] T \mid \\
R_{\mathbf{i j}}^{\mu} & =-864 i \sigma_{\alpha \dot{\alpha}}^{\mu}\left[D_{(\mathbf{i}}^{\alpha}, \bar{D}_{\mathbf{j}}^{\dot{\alpha}}\right] T \mid \\
J_{\alpha}^{\mu \mathbf{i}} & =192\left[-12 \partial^{\mu} \chi_{\alpha}^{\mathbf{i}}-3 i \bar{\sigma}^{\mu \dot{\alpha} \beta} \bar{D}_{\dot{\alpha}}^{\mathbf{i}} D_{\alpha \beta} T \mid-8 \sigma_{\alpha}^{\mu \nu \beta} \partial_{\nu} \chi_{\beta}^{\mathbf{i}}\right], \\
T^{\mu \nu} & =-24\left[-\frac{3}{2} \bar{\sigma}^{\mu \dot{\alpha} \alpha} \bar{\sigma}^{\nu \dot{\beta} \beta}\left\{D_{\alpha \beta}, \bar{D}_{\dot{\alpha} \dot{\beta}}\right\} T \mid+48 \eta^{\mu \nu} \square t-64\left(\eta^{\mu \nu} \square-\partial^{\mu} \partial^{\nu}\right) t\right] .
\end{aligned}
$$

The transformation properties of these components are given by:

$$
\begin{aligned}
\delta_{\alpha \mathbf{i}} t & =\chi_{\alpha \mathbf{i}} \\
\delta_{\alpha \mathbf{i}} \chi_{\beta \mathbf{j}} & =\frac{1}{2} \varepsilon_{\mathbf{i j}} u_{\alpha \beta} \\
\bar{\delta}_{\dot{\alpha} \mathbf{i}} \chi_{\alpha \mathbf{j}} & =\frac{i}{3456} R_{\alpha \dot{\alpha} \mathbf{i j}}+\frac{1}{384} \varepsilon_{\mathbf{i j}} \mathcal{R}_{\alpha \dot{\alpha}}+i \varepsilon_{\mathbf{i j}} \partial_{\alpha \dot{\alpha}} t \\
\delta_{\alpha \mathbf{i}} u_{\beta \gamma} & =0 \\
\bar{\delta}_{\dot{\alpha}}^{\mathbf{i}} u_{\beta \gamma} & \left.=-\frac{i}{1152} \sigma_{\mu(\beta \dot{\alpha}} J_{\gamma)}^{\mu \mathbf{i}}-\frac{8 i}{3} \partial_{(\beta \dot{\alpha}} \chi_{\gamma)}^{\mathbf{i}}\right) \\
\delta_{\alpha \mathbf{i}} \mathcal{R}^{\mu} & =16 i\left[\frac{1}{192} J_{\alpha \mathbf{i}}^{\mu}-16 \sigma_{\alpha}^{\mu \nu} \partial_{\nu} \chi_{\beta \mathbf{i}}\right] \\
\delta_{\alpha \mathbf{i}} R_{\mathbf{j} \mathbf{k}}^{\mu} & =\frac{3}{2} \varepsilon_{\mathbf{i}(\mathbf{j}}\left[J_{\alpha \mathbf{k})}^{\mu}-16 \sigma_{\alpha}^{\mu \nu} \beta \partial_{\nu} \chi_{\beta \mathbf{k})}\right] \\
\bar{\delta}_{\dot{\alpha}}^{\mathbf{i}} J_{\alpha}^{\mu \mathbf{j}} & =\varepsilon^{\mathbf{i j}}\left[-2 i \sigma_{\nu \alpha \dot{\alpha}} T^{\mu \nu}-4 \partial_{\alpha \dot{\alpha}} \mathcal{R}^{\mu}+2 i \varepsilon^{\mu \nu \rho \tau} \sigma_{\tau \alpha \dot{\alpha}} \partial_{\nu} \mathcal{R}_{\rho}\right] \\
& -\frac{8 i}{9} \partial_{\alpha \dot{\alpha}} R^{\mu \mathbf{i j}}-\frac{4 i}{9} \varepsilon^{\mu \nu \rho \tau} \sigma_{\tau \alpha \dot{\alpha}} \partial_{\nu} R_{\rho}^{\mathbf{i j}} \\
\delta_{\alpha \mathbf{i}} J_{\beta \mathbf{j}}^{\mu} & =1536 \varepsilon_{\mathbf{i j}} \partial_{\nu}\left[\varepsilon_{\alpha \beta} \sigma_{\gamma \delta}^{\mu \nu} u^{\gamma \delta}+\sigma_{(\alpha}^{\mu \nu \gamma} u_{\beta) \gamma}\right] \\
\delta_{\alpha \mathbf{i}} T^{\mu \nu} & =\sigma_{\alpha}^{(\mu \rho \beta} \partial_{\rho} J_{\beta \mathbf{i}}^{\nu)} .
\end{aligned}
$$

\section{C $S U(2)_{R}$ Invariance for the $\mathcal{N}=2$ Tensor Multiplet}

In this appendix we sketch the proof of the identity (6.29)

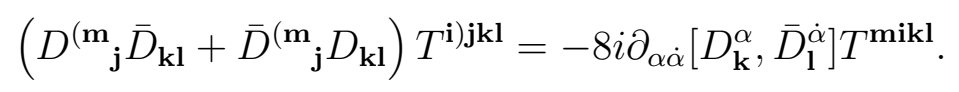

The starting point is to notice that

$$
T^{\mathbf{i j k} \mathbf{l}}=\frac{1}{160} F^{(\mathbf{i j}} F^{\mathbf{k l})}=D^{(\mathbf{i j}} X^{\mathbf{k l})}+c . c . \quad \text { with } \quad X^{\mathbf{i j}} \equiv \frac{i}{160} \Phi F^{\mathbf{i j}} .
$$

As $\Phi$ is chiral, $X^{\mathbf{i j}}$ has clearly the same properties (4.42) than $F^{\mathbf{i j}}$. The first step consists in writing $D^{\mathbf{m}} \bar{D}_{\mathbf{k l}}=\left[D^{\mathbf{m}} \mathbf{j}, \bar{D}_{\mathbf{k l}}\right]+\bar{D}_{\mathbf{k l}} D^{\mathbf{m}_{\mathbf{j}}}$. After some algebra we find

$$
\begin{aligned}
{\left[D_{\mathbf{j}}^{\mathbf{m}}, \bar{D}_{\mathbf{k l}}\right] D^{(\mathbf{i j}} X^{\mathbf{k l})} } & =-4 i \partial_{\alpha \dot{\alpha}} D_{\mathbf{k}}^{\alpha} \bar{D}_{\mathbf{l}}^{\dot{\alpha}} D^{(\mathbf{i m}} X^{\mathbf{k l})} \\
\bar{D}_{\mathbf{k} \mathbf{l}} D^{\mathbf{m}}{ }_{\mathbf{j}} D^{(\mathbf{i j}} X^{\mathbf{k l})} & =\frac{5}{36} \varepsilon^{\mathbf{m i}} \bar{D}_{\mathbf{j k}} D^{4} X^{\mathbf{j k}}+\frac{5}{18} \bar{D}^{\left(\mathbf{m}_{\mathbf{j}}\right.} D^{4} X^{\mathbf{i}) \mathbf{j}} .
\end{aligned}
$$

On the other hand,

$$
\bar{D}_{\mathbf{j}}^{\mathbf{m}} D_{\mathbf{k l}} D^{(\mathbf{i j}} X^{\mathbf{k l})}=\frac{5}{18} \varepsilon^{\mathbf{m i}} \bar{D}_{\mathbf{j k}} D^{4} X^{\mathbf{j k}}+\frac{5}{9} \bar{D}^{\left(\mathbf{m}_{\mathbf{j}}\right.} D^{4} X^{\mathbf{i}) \mathbf{j}}
$$


Adding (

$$
\begin{aligned}
\left(D_{\mathbf{j}}^{\mathbf{m}_{\mathbf{k} \mathbf{l}}}+\bar{D}_{\mathbf{j}} \bar{D}_{\mathbf{k l}}\right) D^{(\mathbf{i j}} X^{\mathbf{k l})}= & \frac{5}{12} \varepsilon^{\mathbf{m i}} \bar{D}_{\mathbf{j k}} D^{4} X^{\mathbf{j k}}+\frac{5}{6} \bar{D}^{\left(\mathbf{m}_{\mathbf{j}}\right.} D^{4} X^{\mathbf{i}) \mathbf{j}} \\
& -4 i \partial_{\alpha \dot{\alpha}} D_{\mathbf{k}}^{\alpha} \bar{D}_{\mathbf{l}}^{\dot{\alpha}} D^{(\mathbf{i m}} X^{\mathbf{k l})}
\end{aligned}
$$

For the second step, we use the fact that

$$
\bar{D}_{\mathbf{j}}^{\left(\mathbf{m}_{\mathbf{j}}\right.} D^{4} X^{\mathbf{i}) \mathbf{j}}=\left[\bar{D}^{(\mathbf{m}} \mathbf{j}, D^{4}\right] X^{\mathbf{i}) \mathbf{j}},
$$

since the other term vanishes due to the properties (4.42) of $X^{\mathbf{i j}}$. Again, we find after some algebra:

$$
\left[\bar{D}^{\left(\mathbf{m}_{\mathbf{j}}\right.}, D^{4}\right] X^{\mathbf{i}) \mathbf{j}}=48 \square D^{\left(\mathbf{m}_{\mathbf{j}}\right.} X^{\mathbf{i}) \mathbf{j}}+\frac{32 i}{5} \partial_{\alpha \dot{\alpha}} D^{3 \alpha(\mathbf{i}} \bar{D}_{\mathbf{j}}^{\dot{\alpha}} X^{\mathbf{m}) \mathbf{j}}
$$

However, a direct computation shows also that

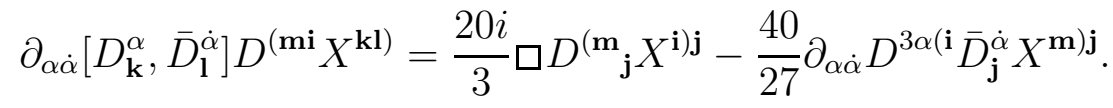

Thus we have proved that

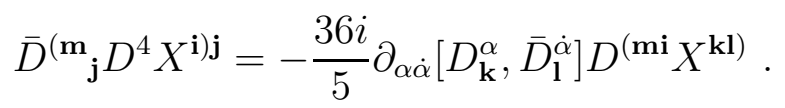

To conclude, we put the results (C.6) and (C.7) together. This leads to the final relation:

$$
\left(D^{\mathbf{m}} \overline{\mathbf{j}}_{\mathbf{k} \mathbf{l}}+\bar{D}^{\mathbf{m}}{ }_{\mathbf{j}} D_{\mathbf{k l}}\right) D^{(\mathbf{i j}} X^{\mathbf{k l})}=\frac{5}{12} \varepsilon^{\mathbf{m i}} \bar{D}^{\mathbf{j k}} D^{4} X_{\mathbf{j k}}-8 i \partial_{\alpha \dot{\alpha}}\left[D_{\mathbf{k}}^{\alpha}, \bar{D}_{\mathbf{l}}^{\dot{\alpha}}\right] D^{(\mathbf{m i}} X^{\mathbf{k l})}
$$

from which we get (C.1) as a consequence.

\section{Conventions and Identities for the SUSY Alge- bra}

The conventions used in this paper are essentially those of Wess and Bagger [31. The conventions about covariant derivatives and their algebra in $\mathcal{N}=1$ as well as in extended supersymmetry are exposed in this appendix.

The following definitions are valid for any $\mathcal{N}$. The spinorial derivatives are defined by

$$
\partial_{\beta}^{\mathbf{j}} \theta_{\mathbf{i}}^{\alpha} \equiv \frac{\partial \theta_{\mathbf{i}}^{\alpha}}{\partial \theta_{\mathbf{j}}^{\beta}}=\delta_{\beta}^{\alpha} \delta_{\mathbf{i}}^{\mathbf{j}}, \quad \quad \bar{\partial}_{\dot{\beta} \mathbf{j}} \bar{\theta}^{\dot{\alpha} \mathbf{i}} \equiv \frac{\partial \bar{\theta}^{\dot{\alpha} \mathbf{i}}}{\partial \bar{\theta}_{\dot{\beta} \mathbf{j}}}=\delta_{\dot{\beta}}^{\dot{\alpha}} \delta_{\mathbf{j}}^{\mathbf{i}} .
$$

The covariant derivative are then defined as

$$
D_{\alpha}^{\mathbf{i}}=\partial_{\alpha}^{\mathbf{i}}+i \bar{\theta}^{\dot{\alpha} \mathbf{i}} \partial_{\alpha \dot{\alpha}}, \quad \bar{D}_{\dot{\alpha} \mathbf{i}}=-\bar{\partial}_{\dot{\alpha} \mathbf{i}}-i \theta_{\dot{\mathbf{i}}}^{\alpha} \partial_{\alpha \dot{\alpha}}
$$

Finally, the algebra following from these definitions is

$$
\left\{D_{\alpha}^{\mathbf{i}}, \bar{D}_{\dot{\alpha} \mathbf{j}}\right\}=-2 i \delta_{\mathbf{j}}^{\mathbf{i}} \partial_{\alpha \dot{\alpha}} \quad \text { and } \quad\left\{D_{\alpha}^{\mathbf{i}}, D_{\beta}^{\mathbf{j}}\right\}=0 .
$$


We furthermore use the following usual definition of $x_{ \pm}^{\mu}: x_{ \pm}^{\alpha \dot{\alpha}} \equiv x^{\alpha \dot{\alpha}} \pm 2 i \theta^{\alpha i} \bar{\theta}_{i}^{\dot{\alpha}}$. The main properties of $x_{ \pm}^{\mu}$ with respect to the covariant derivatives are:

$$
\begin{gathered}
\bar{D}_{\alpha \mathbf{i}} x_{+}^{\mu}=0, \quad D_{\alpha}^{\mathbf{i}} x_{-}^{\mu}=0 \\
D_{\alpha \mathbf{i}} x_{+\beta \dot{\beta}}=4 i \varepsilon_{\alpha \beta} \bar{\theta}_{\dot{\beta} \mathbf{i}}, \quad \bar{D}_{\dot{\alpha} \mathbf{i}} x_{-\beta \dot{\beta}}=-4 i \varepsilon_{\dot{\alpha} \dot{\beta}} \theta_{\beta \mathbf{i}} .
\end{gathered}
$$

The different integration measures are then defined by:

$\int d^{4+4 \mathcal{N}} z \equiv \int d^{4} x D^{2 \mathcal{N}} \bar{D}^{2 \mathcal{N}}, \quad \int d^{4+2 \mathcal{N}} z_{+} \equiv \int d^{4} x D^{2 \mathcal{N}}, \quad \int d^{4+2 \mathcal{N}} z_{-} \equiv \int d^{4} x \bar{D}^{2 \mathcal{N}}$,

where $D^{2 \mathcal{N}}$ is defined below for the specific values $\mathcal{N}=1,2$.

\section{D.1 $\mathcal{N}=1$ Identities}

We give there a list of useful identities for $\mathcal{N}=1$ covariant derivatives following from the definitions and conventions given above.

Definition of $D^{2}$ :

$$
D^{2} \equiv D^{\alpha} D_{\alpha}, \quad \bar{D}^{2} \equiv \bar{D}_{\dot{\alpha}} \bar{D}^{\dot{\alpha}} .
$$

Products of covariant derivatives:

$$
\begin{gathered}
D^{\alpha} \bar{D}^{2} D_{\alpha}=\bar{D}_{\dot{\alpha}} D^{2} \bar{D}^{\dot{\alpha}} \\
D^{\alpha} \bar{D}_{\dot{\alpha}} D_{\alpha}=-\frac{1}{2} \bar{D}_{\dot{\alpha}} D^{2}-\frac{1}{2} D^{2} \bar{D}_{\dot{\alpha}}, \quad \bar{D}_{\dot{\alpha}} D^{\alpha} \bar{D}^{\dot{\alpha}}=-\frac{1}{2} D^{\alpha} \bar{D}^{2}-\frac{1}{2} \bar{D}^{2} D^{\alpha}, \\
{\left[D^{\alpha}, \bar{D}^{\dot{\alpha}}\right]\left[D_{\alpha}, \bar{D}_{\dot{\alpha}}\right]=2\left\{D^{2}, \bar{D}^{2}\right\}-24 \square .}
\end{gathered}
$$

Algebra of covariant derivatives:

$$
\begin{gathered}
{\left[D_{\alpha}, \bar{D}^{2}\right]=-4 i \partial_{\alpha \dot{\alpha}} \bar{D}^{\dot{\alpha}}, \quad\left[\bar{D}_{\dot{\alpha}}, D^{2}\right]=4 i D^{\alpha} \partial_{\alpha \dot{\alpha}}} \\
{\left[D^{2}, \bar{D}^{2}\right]=-4 i\left[D^{\alpha}, \bar{D}^{\dot{\alpha}}\right] \partial_{\alpha \dot{\alpha}}}
\end{gathered}
$$

\section{D.2 $\mathcal{N}=2$ Identities}

Raising and lowering of $S U(2)$ indices and Fierz formula:

$$
a^{\mathbf{i}}=\varepsilon^{\mathbf{i j}} a_{\mathbf{j}}, \quad a_{\mathbf{i}}=\varepsilon_{\mathbf{i j}} a^{\mathbf{j}},
$$

with $\quad \varepsilon^{\mathbf{i j}}=-\varepsilon^{\mathbf{j i}}, \quad \varepsilon^{\mathbf{1 2}}=1, \quad \varepsilon_{\mathbf{i j}}=-\varepsilon^{\mathbf{i j}}, \quad \varepsilon^{\mathbf{i} \mathbf{j}} \varepsilon_{\mathbf{j k}}=\delta_{\mathbf{k}}^{\mathbf{i}}$.

$$
a_{\mathbf{i}} b_{\mathbf{j}}-a_{\mathbf{j}} b_{\mathbf{i}}=\varepsilon_{\mathbf{i j}} a^{\mathbf{k}} b_{\mathbf{k}}, \quad a^{\mathbf{i}} b^{\mathbf{j}}-a^{\mathbf{j}} b^{\mathbf{i}}=-\varepsilon^{\mathbf{i} \mathbf{j}} a^{\mathbf{k}} b_{\mathbf{k}} .
$$

Definitions of some products of $\theta$ 's:

$$
\begin{array}{ccc}
\theta^{2}: & \theta^{\mathbf{i j}} \equiv \theta^{\alpha \mathbf{i}} \theta_{\alpha}^{\mathbf{j}}, \quad \theta^{\alpha \beta} \equiv \theta^{\alpha \mathbf{i}} \theta_{\mathbf{i}}^{\beta}, & \bar{\theta}^{\mathbf{i j}} \equiv \bar{\theta}_{\dot{\alpha}}^{\mathbf{i}} \bar{\theta}^{\dot{\alpha} \mathbf{j}}, \quad \bar{\theta}^{\dot{\alpha} \dot{\beta}} \equiv \bar{\theta}^{\dot{\alpha} \mathbf{i}} \bar{\theta}_{\mathbf{i}}^{\dot{\beta}} \\
\theta^{3}: & \theta_{\alpha \mathbf{i}}^{3} \equiv \theta_{\alpha}^{\mathbf{j}} \theta_{\mathbf{i j}}=-\theta_{\mathbf{i}}^{\beta} \theta_{\alpha \beta}, \quad \bar{\theta}_{\dot{\alpha}}^{3 \mathbf{i}} \equiv-\bar{\theta}_{\dot{\alpha} \mathbf{j}} \bar{\theta}^{\mathbf{i j}}=\bar{\theta}^{\dot{\beta} \mathbf{i}} \bar{\theta}_{\dot{\alpha} \dot{\beta}} . \\
\theta^{4}: & \theta^{4} \equiv \theta^{\mathbf{i j}} \theta_{\mathbf{i j}}=-\theta^{\alpha \beta} \theta_{\alpha \beta}, \quad \bar{\theta}^{4} \equiv \bar{\theta}^{\mathbf{i j}} \bar{\theta}_{\mathbf{i} \mathbf{j}}=-\bar{\theta}^{\dot{\alpha} \dot{\beta}} \bar{\theta}_{\dot{\alpha} \dot{\beta}} .
\end{array}
$$


Properties of the products of $\theta$ 's:

$$
\begin{aligned}
& \theta_{\alpha \mathbf{i}} \theta_{\beta \gamma}=-\frac{2}{3} \varepsilon_{\alpha(\beta} \theta_{\gamma) \mathbf{i}}^{3}, \quad \theta_{\alpha \mathbf{i}} \theta_{\mathbf{j k}}=\frac{2}{3} \varepsilon_{\mathbf{i}(\mathbf{j}} \theta_{\alpha \mathbf{k})}^{3}, \quad \bar{\theta}_{\dot{\alpha} \mathbf{i}} \bar{\theta}_{\dot{\beta} \dot{\gamma}}=\frac{2}{3} \varepsilon_{\dot{\alpha}(\dot{\beta}} \bar{\theta}_{\dot{\gamma}) \mathbf{i}}^{3}, \quad \bar{\theta}_{\dot{\alpha} \mathbf{i}} \bar{\theta}_{\mathbf{j k}}=\frac{2}{3} \varepsilon_{\mathbf{i}(\mathbf{j}} \bar{\theta}_{\dot{\alpha} \mathbf{k})}^{3}, \\
& \theta^{\beta \mathbf{j}} \theta_{\alpha \mathbf{i}}^{3}=\frac{1}{4} \delta_{\alpha}^{\beta} \delta_{\mathbf{i}}^{\mathbf{j}} \theta^{4}, \quad \theta^{\mathbf{i j}} \theta^{\alpha \beta}=0, \quad \theta^{\mathbf{i j}} \theta^{\mathbf{k l}}=\frac{1}{3} \varepsilon^{(\mathbf{i} \mid \mathbf{l}} \varepsilon^{\mid \mathbf{j}) \mathbf{k}} \theta^{4}, \quad \theta^{\alpha \beta} \theta^{\gamma \delta}=-\frac{1}{3} \varepsilon^{(\alpha \mid \gamma} \varepsilon^{\mid \beta) \delta} \theta^{4}, \\
& \bar{\theta}_{\dot{\mathbf{j}}}^{\dot{\beta}} \bar{\theta}_{\dot{\alpha}}^{3 \mathbf{i}}=-\frac{1}{4} \delta_{\dot{\alpha}}^{\dot{\beta}} \delta_{\mathbf{j}}^{\mathbf{i}} \bar{\theta}^{4}, \quad \bar{\theta}^{\mathbf{i j}} \bar{\theta}^{\dot{\alpha} \dot{\beta}}=0, \bar{\theta}^{\mathbf{i j}} \bar{\theta}^{\mathbf{k l}}=\frac{1}{3} \varepsilon^{(\mathbf{i} \mid \mathbf{l}} \varepsilon^{\mid \mathbf{j}) \mathbf{k}} \bar{\theta}^{4}, \quad \bar{\theta}^{\dot{\alpha} \dot{\beta}} \bar{\theta}^{\dot{\gamma} \dot{\delta}}=-\frac{1}{3} \varepsilon^{(\dot{\alpha} \mid \dot{\gamma}} \varepsilon^{\mid \dot{\beta}) \dot{\delta}} \bar{\theta}^{4} .
\end{aligned}
$$

Definitions of products of covariant derivatives:

$$
\begin{array}{ccc}
D^{2}: & D^{\mathbf{i j}} \equiv D^{\alpha \mathbf{i}} D_{\alpha}^{\mathbf{j}}, \quad D^{\alpha \beta} \equiv D^{\alpha \mathbf{i}} D_{\mathbf{i}}^{\beta}, & \bar{D}^{\mathbf{i j}} \equiv \bar{D}_{\dot{\alpha}}^{\mathbf{i}} \bar{D}^{\dot{\alpha} \mathbf{j}}, \quad \bar{D}^{\dot{\alpha} \dot{\beta}} \equiv \bar{D}^{\dot{\alpha} \mathbf{i}} \bar{D}_{\mathbf{i}}^{\dot{\beta}} \\
D^{3}: & D_{\alpha \mathbf{i}}^{3} \equiv D_{\alpha}^{\mathbf{j}} D_{\mathbf{j i}}=-D_{\mathbf{i}}^{\beta} D_{\beta \alpha}, & \bar{D}_{\dot{\alpha}}^{3 \mathbf{i}} \equiv \bar{D}_{\dot{\alpha} \mathbf{j}} \bar{D}^{\mathbf{i j}}=-\bar{D}^{\dot{\beta} \mathbf{i}} \bar{D}_{\dot{\beta} \dot{\alpha}} \\
D^{4}: & D^{4} \equiv D^{\mathbf{i j}} D_{\mathbf{i j}}=-D^{\alpha \beta} D_{\alpha \beta}, & \bar{D}^{4} \equiv \bar{D}^{\mathbf{i j}} \bar{D}_{\mathbf{i j}}=-\bar{D}^{\dot{\alpha} \dot{\beta}} \bar{D}_{\dot{\alpha} \dot{\beta}}
\end{array}
$$

Properties of the products of covariant derivatives:

$$
\begin{array}{ll}
(12): & D_{\mathbf{i}}^{\alpha} D_{\mathbf{j} \mathbf{k}}=\frac{2}{3} \varepsilon_{\mathbf{i}(\mathbf{j}} D_{\mathbf{k})}^{3 \alpha}, \quad \bar{D}_{\mathbf{i}}^{\dot{\alpha}} \bar{D}_{\mathbf{j} \mathbf{k}}=-\frac{2}{3} \varepsilon_{\mathbf{i}(\mathbf{j}} \bar{D}_{\mathbf{k})}^{3 \dot{\alpha}} \\
& D_{\alpha \mathbf{i}} D_{\beta \gamma}=-\frac{2}{3} \varepsilon_{\alpha(\beta} D_{\gamma) \mathbf{i}}^{3}, \quad \bar{D}_{\dot{\alpha} \mathbf{i}} \bar{D}_{\dot{\beta} \dot{\gamma}}=-\frac{2}{3} \varepsilon_{\dot{\alpha}(\dot{\beta}} \bar{D}_{\dot{\gamma}) \mathbf{i}}^{3}, \\
(13): & D_{\alpha \mathbf{i}} D_{\beta \mathbf{j}}^{3}=\frac{1}{4} \varepsilon_{\alpha \beta} \varepsilon_{\mathbf{i j}} D^{4}, \quad \bar{D}_{\dot{\alpha} \mathbf{i}} \bar{D}_{\dot{\beta} \mathbf{j}}^{3}=\frac{1}{4} \varepsilon_{\dot{\alpha} \dot{\beta}} \varepsilon_{\mathbf{i j}} \bar{D}^{4}, \\
(22): & D_{\mathbf{i j}} D_{\mathbf{k} \mathbf{l}}=\frac{1}{3} \varepsilon_{(\mathbf{i} \mid \mathbf{l}} \varepsilon_{\mid \mathbf{j}) \mathbf{k}} D^{4}, \quad \bar{D}_{\mathbf{i} \mathbf{j}} \bar{D}_{\mathbf{k} \mathbf{l}}=\frac{1}{3} \varepsilon_{(\mathbf{i} \mid \mathbf{l}} \varepsilon_{\mid \mathbf{j}) \mathbf{k}} \bar{D}^{4}, \\
& D_{\mathbf{i} \mathbf{j}} D_{\alpha \beta}=0, \quad \bar{D}_{\mathbf{i} \mathbf{j}} \bar{D}_{\dot{\alpha} \dot{\beta}}=0, \quad D_{\mathbf{i j}} \bar{D}^{4} D_{\mathbf{k} \mathbf{l}}=\bar{D}_{\mathbf{i j}} D^{4} \bar{D}_{\mathbf{k} \mathbf{l}} .
\end{array}
$$

\section{D.2.1 Complex Conjugation}

The general complex conjugation rule for the $S U(2)$ indices is the following: $\left(\varphi_{\mathbf{i}}\right)^{*}=$ $\bar{\varphi}^{\mathbf{i}}$. As a consequence of this, we have $\left(\bar{\varphi}_{\mathbf{i}}\right)^{*}=\left(\varepsilon_{\mathbf{i j}} \bar{\varphi}^{\mathbf{j}}\right)^{*}=\varepsilon_{\mathbf{i j}} \varphi_{\mathbf{j}}=-\varepsilon^{\mathbf{i} \mathbf{j}} \varphi_{\mathbf{j}}=-\varphi^{\mathbf{i}}$. For the specific products of $\theta$ 's defined above, this leads to:

$$
\left(\theta^{\mathbf{i j}}\right)^{*}=\bar{\theta}_{\mathbf{i j}}, \quad\left(\theta^{\alpha \beta}\right)^{*}=-\bar{\theta}^{\dot{\alpha} \dot{\beta}}, \quad\left(\theta^{\alpha \dot{\alpha}}\right)^{*}=\theta^{\alpha \dot{\alpha}}, \quad\left(\theta_{\alpha \mathbf{i}}^{3}\right)^{*}=\bar{\theta}_{\dot{\alpha}}^{3 \mathbf{i}}, \quad\left(\theta^{4}\right)^{*}=\bar{\theta}^{4} .
$$

For the spinorial and covariant derivatives, this gives:

$$
\begin{array}{ll}
\left(\partial_{\alpha}^{\mathbf{i}}\right)^{*}=-\bar{\partial}_{\dot{\alpha} \mathbf{i}}, & \left(D_{\alpha}^{\mathbf{i}}\right)^{*}=\bar{D}_{\dot{\alpha} \mathbf{i}}, \\
\left(\partial_{\alpha \mathbf{i}}\right)^{*}=\bar{\partial}_{\dot{\alpha}}^{\mathbf{i}}, & \left(D_{\alpha \mathbf{i}}\right)^{*}=-\bar{D}_{\dot{\alpha}}^{\mathbf{i}},
\end{array}
$$

and for higher order products:

$$
\left(D^{\mathbf{i j}}\right)^{*}=\bar{D}_{\mathbf{i j}}, \quad\left(D^{\alpha \beta}\right)^{*}=-\bar{D}^{\dot{\alpha} \dot{\beta}}, \quad\left(D_{\alpha \mathbf{i}}^{3}\right)^{*}=\bar{D}_{\dot{\alpha}}^{3 \mathbf{i}}, \quad\left(D^{4}\right)^{*}=\bar{D}^{4} .
$$

\section{D.2.2 Algebra of covariant Derivatives}




$$
\begin{aligned}
{\left[D_{\mathbf{i j}}, \bar{D}_{\dot{\alpha} \mathbf{k}}\right] } & =4 i \varepsilon_{\mathbf{k}(\mathbf{i}} D_{\mathbf{j})}^{\alpha} \partial_{\alpha \dot{\alpha}}, \\
{\left[\bar{D}_{\mathbf{i j}}, D_{\alpha \mathbf{k}}\right] } & =4 i \varepsilon_{\mathbf{k}(\mathbf{i}} \partial_{\alpha \dot{\alpha}} \bar{D}_{\mathbf{j})}^{\dot{\alpha}}, \\
{\left[D_{\alpha \beta}, \bar{D}_{\dot{\alpha} \mathbf{i}}\right] } & =4 i \partial_{(\alpha \dot{\alpha}} D_{\beta) \mathbf{i}}, \\
{\left[\bar{D}_{\dot{\alpha} \dot{\beta}}, D_{\alpha \mathbf{i}}\right] } & =-4 i \partial_{\alpha(\dot{\alpha}} \bar{D}_{\dot{\beta}) \mathbf{i}}, \\
{\left[D_{\mathbf{i j}}, \bar{D}_{\dot{\alpha}}^{\mathbf{j}}\right] } & =6 i D_{\mathbf{i}}^{\alpha} \partial_{\alpha \dot{\alpha}}, \\
{\left[\bar{D}_{\mathbf{i j}}, D_{\alpha}^{\mathbf{j}}\right] } & =6 i \partial_{\alpha \dot{\alpha}} \bar{D}_{\mathbf{i}}^{\dot{\alpha}}
\end{aligned}
$$

(22) :

$$
\begin{aligned}
{\left[D_{\mathbf{i j}}, \bar{D}_{\mathbf{k} \mathbf{l}}\right] } & =16 \varepsilon_{\mathbf{k}(\mathbf{i}} \varepsilon_{\mathbf{j}) \mathbf{l}} \square-8 i \varepsilon_{(\mathbf{i}(\mathbf{k}} D_{\mathbf{j})}^{\alpha} \partial_{\alpha \dot{\alpha}} \bar{D}_{\mathbf{l})}^{\dot{\alpha}}, \\
& =-16 \varepsilon_{\mathbf{k}(\mathbf{i}} \varepsilon_{\mathbf{j}) 1} \square+8 i \varepsilon_{(\mathbf{i}(\mathbf{k}} \bar{D}_{\mathbf{l})}^{\dot{\alpha}} \partial_{\alpha \dot{\alpha}} D_{\mathbf{j})}^{\alpha}, \\
{\left[D_{\mathbf{i j}}, \bar{D}_{\dot{\alpha} \dot{\beta}}\right] } & =8 i \partial_{\alpha(\dot{\alpha}} D_{(\mathbf{i}}^{\alpha} \bar{D}_{\dot{\beta}) \mathbf{j})}, \\
{\left[\bar{D}_{\mathbf{i j}}, D_{\alpha \beta}\right] } & =-8 i \partial_{(\alpha \dot{\alpha}} D_{\beta)(\mathbf{i}} \bar{D}_{\mathbf{j})}^{\dot{\alpha}}, \\
{\left[D_{\alpha \beta}, \bar{D}_{\dot{\alpha} \dot{\beta}}\right] } & =4 i \partial_{(\alpha(\dot{\alpha}}\left[D_{\beta)}^{\mathbf{i}}, \bar{D}_{\dot{\beta}) \mathbf{i}}\right], \\
{\left[D_{\mathbf{i}}^{\mathbf{j}}, \bar{D}_{\mathbf{j k}}\right] } & =-4 i \partial_{\alpha \dot{\alpha}}\left[D_{\mathbf{i}}^{\alpha}, \bar{D}_{\mathbf{k}}^{\dot{\alpha}}\right]-i \varepsilon_{\mathbf{i k}} \partial_{\alpha \dot{\alpha}}\left[D^{\alpha \mathbf{j}}, \bar{D}_{\mathbf{j}}^{\dot{\alpha}}\right], \\
{\left[D^{\mathbf{i j}}, \bar{D}_{\mathbf{i j}}\right] } & =-6 i \partial_{\alpha \dot{\alpha}}\left[D^{\alpha \mathbf{i}}, \bar{D}_{\mathbf{i}}^{\dot{\alpha}}\right],
\end{aligned}
$$

(13) :

$$
\begin{aligned}
& \left\{D_{\alpha \mathbf{i}}, \bar{D}_{\dot{\alpha} \mathbf{j}}^{3}\right\}=-3 i \varepsilon_{\mathbf{i j}} \partial_{\alpha \dot{\beta}} \bar{D}_{\dot{\alpha}}^{\dot{\beta}}-3 i \partial_{\alpha \dot{\alpha}} \bar{D}_{\mathbf{i j}} \\
& \left\{\bar{D}_{\dot{\alpha} \mathbf{i}}, D_{\alpha \mathbf{j}}^{3}\right\}=3 i \varepsilon_{\mathbf{i j}} D_{\alpha}{ }^{\beta} \partial_{\beta \dot{\alpha}}-3 i \partial_{\alpha \dot{\alpha}} D_{\mathbf{i j}} .
\end{aligned}
$$

(14) :

$$
\begin{aligned}
{\left[D^{\alpha \mathbf{i}}, \bar{D}^{4}\right] } & =8 i \partial^{\alpha \dot{\alpha}} \bar{D}_{\dot{\alpha}}^{3 \mathbf{i}} \\
{\left[\bar{D}_{\dot{\alpha}}^{\mathbf{i}}, D^{4}\right] } & =8 i D^{3 \alpha \mathbf{i}} \partial_{\alpha \dot{\alpha}} .
\end{aligned}
$$

(23) :

$$
\begin{aligned}
{\left[D_{\mathbf{k}}^{3 \alpha}, \bar{D}_{\mathbf{i j}}\right] } & =\varepsilon_{\mathbf{k}(\mathbf{i}}\left(-24 D_{\mathbf{j})}^{\alpha} \square-6 i \bar{D}_{\mathbf{j})}^{\dot{\beta}} D^{\alpha \beta} \partial_{\beta \dot{\beta}}\right)+6 i \partial^{\alpha \dot{\alpha}} \bar{D}_{\dot{\alpha}(\mathbf{i}} D_{\mathbf{j}) \mathbf{k}}, \\
{\left[\bar{D}_{\mathbf{k}}^{3 \dot{\alpha}}, D_{\mathbf{i j}}\right] } & =\varepsilon_{\mathbf{k}(\mathbf{i}}\left(24 \bar{D}_{\mathbf{j})}^{\dot{\alpha}} \square-6 i D_{\mathbf{j})}^{\beta} \bar{D}^{\dot{\alpha} \dot{\beta}} \partial_{\beta \dot{\beta}}\right)-6 i \partial^{\alpha \dot{\alpha}} D_{\alpha(\mathbf{i}} \bar{D}_{\mathbf{j}) \mathbf{k}}, \\
{\left[D_{\mathbf{k}}^{3 \alpha}, \bar{D}_{\dot{\alpha} \dot{\beta}}\right] } & =-24 \partial^{\alpha}{ }_{(\dot{\alpha}} \partial_{\beta \dot{\beta}} D_{\mathbf{k}}^{\beta}+6 i \bar{D}_{(\dot{\alpha} \mathbf{k}} D^{\alpha \beta} \partial_{\beta \dot{\beta})}+6 i \partial^{\alpha}{ }_{(\dot{\alpha}} \bar{D}_{\dot{\beta})}^{\mathbf{i}} D_{\mathbf{i k}}, \\
{\left[\bar{D}_{\mathbf{k}}^{3 \dot{\alpha}}, D_{\alpha \beta}\right] } & =-24 \partial_{(\alpha \dot{\beta}} \partial_{\beta)}{ }^{\dot{\alpha}} \bar{D}_{\mathbf{k}}^{\dot{\beta}}-6 i D_{(\alpha \mathbf{k}} \bar{D}^{\dot{\alpha} \dot{\beta}} \partial_{\beta) \dot{\beta}}+6 i \partial_{(\alpha}{ }^{\dot{\alpha}} D_{\beta)}^{\mathbf{i}} \bar{D}_{\mathbf{i k}}, \\
{\left[D_{\mathbf{j}}^{3 \alpha}, \bar{D}^{\mathbf{i j}}\right] } & =36 \square D^{\alpha \mathbf{i}}+9 i \partial_{\beta \dot{\alpha}} \bar{D}^{\dot{\alpha} \mathbf{i}} D^{\alpha \beta}-3 i \partial^{\alpha \dot{\alpha}} \bar{D}_{\dot{\alpha} \mathbf{j}} D^{\mathbf{i j}}, \\
{\left[\bar{D}_{\mathbf{j}}^{3 \dot{\alpha}}, D^{\mathbf{i j}}\right] } & =-36 \square \bar{D}^{\dot{\alpha} \mathbf{i}}-9 i \partial^{\alpha}{ }_{\dot{\beta}} D_{\alpha}^{\mathbf{i}} \bar{D}^{\dot{\alpha} \dot{\beta}}+3 i \partial^{\alpha \dot{\alpha}} D_{\alpha \mathbf{j}} \bar{D}^{\mathbf{i j} \mathbf{j}} .
\end{aligned}
$$


(24) :

$$
\begin{aligned}
{\left[D_{\mathbf{i j}}, \bar{D}^{4}\right] } & =48 \square \bar{D}_{\mathbf{i} \mathbf{j}}+16 i \partial_{\alpha \dot{\alpha}} \bar{D}_{(\mathbf{i}}^{3 \dot{\alpha}} D_{\mathbf{j})}^{\alpha}, \\
& =-48 \square \bar{D}_{\mathbf{i j}}-16 i \partial_{\alpha \dot{\alpha}} D_{(\mathbf{i}}^{\alpha} \bar{D}_{\mathbf{j})}^{3 \dot{\alpha}}, \\
{\left[D_{\alpha \beta}, \bar{D}^{4}\right] } & =-48 \partial_{(\alpha \dot{\alpha}} \partial_{\beta) \dot{\beta}} \bar{D}^{\dot{\alpha} \dot{\beta}}+16 i \partial_{(\alpha \dot{\alpha}} \bar{D}_{\mathbf{i}}^{3 \dot{\alpha}} D_{\beta)}^{\mathbf{i}}, \\
& =48 \partial_{(\alpha \dot{\alpha}} \partial_{\beta) \dot{\beta}} \bar{D}^{\dot{\alpha} \dot{\beta}}-16 i \partial_{(\alpha \dot{\alpha}} D_{\beta)}^{\mathbf{i}} \bar{D}_{\mathbf{i}}^{3 \dot{\alpha}}, \\
{\left[\bar{D}_{\mathbf{i j}}, D^{4}\right] } & =48 \square D_{\mathbf{i} \mathbf{j}}+16 i \partial_{\alpha \dot{\alpha}} D_{(\mathbf{i}}^{3 \alpha} \bar{D}_{\mathbf{j})}^{\dot{\alpha}}, \\
& =-48 \square D_{\mathbf{i j}}-16 i \partial_{\alpha \dot{\alpha}} \bar{D}_{(\mathbf{i}}^{\dot{\alpha}} D_{\mathbf{j})}^{3 \alpha}, \\
{\left[\bar{D}_{\dot{\alpha} \dot{\beta}}, D^{4}\right] } & =-48 \partial_{\alpha(\dot{\alpha}} \partial_{\beta \dot{\beta})} D^{\alpha \beta}-16 i \partial_{\alpha(\dot{\alpha}} D_{\mathbf{i}}^{3 \alpha} \bar{D}_{\dot{\beta})}^{\mathbf{i}}, \\
& =48 \partial_{\alpha(\dot{\alpha}} \partial_{\beta \dot{\beta})} D^{\alpha \beta}+16 i \partial_{\alpha(\dot{\alpha}} \bar{D}_{\dot{\beta})}^{\mathbf{i}} D_{\mathbf{i}}^{3 \alpha} .
\end{aligned}
$$


(33) :

$$
\begin{aligned}
\left\{D_{\alpha}^{3 \mathbf{i}}, \bar{D}_{\dot{\alpha} \mathbf{i}}^{3}\right\} & =-72 i \partial_{\alpha \dot{\alpha}} \square+\frac{9 i}{4} \partial_{\alpha \dot{\alpha}}\left\{D^{\mathbf{i j}}, \bar{D}_{\mathbf{i j}}\right\}-\frac{9 i}{2} \partial^{\beta \dot{\beta}}\left\{D_{\alpha \beta}, \bar{D}_{\dot{\alpha} \dot{\beta}}\right\} \\
\left\{D_{\alpha(\mathbf{i}}^{3}, \bar{D}_{\dot{\alpha} \mathbf{j})}^{3}\right\} & =-\frac{9 i}{4}\left(\partial_{\alpha \dot{\alpha}}\left\{D_{\mathbf{k}(\mathbf{i}}, \bar{D}_{\mathbf{j})}^{\mathbf{k}}\right\}+\partial_{\beta \dot{\alpha}}\left\{D_{\alpha}{ }^{\beta}, \bar{D}_{\mathbf{i j}}\right\}+\partial_{\alpha \dot{\beta}}\left\{D_{\mathbf{i j}}, \bar{D}_{\dot{\alpha}}^{\dot{\beta}}\right\}\right)
\end{aligned}
$$

(34) :

$$
\begin{aligned}
{\left[D_{\alpha \mathbf{i}}^{3}, \bar{D}^{4}\right]=} & -288 i \bar{D}_{\mathbf{i}}^{\dot{\alpha}} \partial_{\alpha \dot{\alpha}} \square-72 D_{\alpha}^{\mathbf{j}} \bar{D}_{\mathbf{i j}} \square-72 \partial_{\alpha \dot{\alpha}} \partial_{\beta \dot{\beta}} D_{\mathbf{i}}^{\beta} \bar{D}^{\dot{\alpha} \dot{\beta}} \\
& -12 i \partial_{\alpha \dot{\alpha}} D_{\mathbf{i} \mathbf{j}} \bar{D}^{3 \dot{\mathbf{j}} \mathbf{j}}-12 i \partial^{\beta \dot{\beta}} D_{\alpha \beta} \bar{D}_{\dot{\beta} \mathbf{i}}^{3} \\
{\left[\bar{D}_{\dot{\alpha} \mathbf{i}}^{3}, D^{4}\right]=} & 288 i D_{\mathbf{i}}^{\alpha} \partial_{\alpha \dot{\alpha}} \square+72 \bar{D}_{\dot{\alpha}}^{\mathbf{j}} D_{\mathbf{i j} \mathbf{j}} \square-72 \partial_{\alpha \dot{\alpha}} \partial_{\beta \dot{\beta}} \bar{D}_{\mathbf{i}}^{\dot{\beta}} D^{\alpha \beta} \\
& -12 i \partial_{\alpha \dot{\alpha}} \bar{D}_{\mathbf{i j}} D^{3 \alpha \mathbf{j}}+12 i \partial^{\beta \dot{\beta}} \bar{D}_{\dot{\alpha} \dot{\beta}} D_{\beta \mathbf{i}}^{3} .
\end{aligned}
$$

(44) :

$$
\left[D^{4}, \bar{D}^{4}\right]=-16 i \partial_{\alpha \dot{\alpha}}\left[D^{3 \alpha \mathbf{i}}, \bar{D}_{\mathbf{i}}^{3 \dot{\alpha}}\right]+288 i \square \partial_{\alpha \dot{\alpha}}\left[D^{\alpha \mathbf{i}}, \bar{D}_{\mathbf{i}}^{\dot{\alpha}}\right]
$$

\section{D.2.3 Derivatives acting on $\theta$}

$$
\begin{aligned}
& \partial_{\alpha \mathbf{i}} \theta^{4}=-4 \theta_{\alpha \mathbf{i}}^{3}, \quad \bar{\partial}_{\dot{\alpha}}^{\mathbf{i}} \bar{\theta}^{4}=-4 \bar{\theta}_{\dot{\alpha}}^{3 \mathbf{i}}, \\
& \partial_{\alpha \mathbf{i}} \theta^{3 \beta \mathbf{j}}=-\frac{3}{2}\left(\delta_{\alpha}^{\beta} \theta_{\mathbf{i}}^{\mathbf{j}}-\delta_{\mathbf{i}}^{\mathbf{j}} \theta_{\alpha}^{\beta}\right), \quad \bar{\partial}_{\dot{\alpha}}^{\mathbf{i}} \bar{\theta}_{\mathbf{j}}^{3 \dot{\beta}}=-\frac{3}{2}\left(\delta_{\mathbf{j}}^{\mathbf{i}} \dot{\theta}_{\dot{\alpha}}^{\dot{\beta}}-\delta_{\dot{\alpha}}^{\dot{\beta}} \bar{\theta}_{\mathbf{j}}^{\mathbf{i}}\right), \\
& D^{\mathbf{i j}} \theta^{4}=-12 \theta^{\mathbf{i j}}, \quad D_{\mathbf{i j}} \theta^{3 \alpha \mathbf{k}}=6 \delta_{(\mathbf{i}}^{\mathbf{k}} \theta_{\mathbf{j})}^{\alpha}, \quad D^{\mathbf{i j}} \theta_{\alpha \mathbf{j}}^{3}=-9 \theta_{\alpha}^{\mathbf{i}}, \\
& D^{\mathbf{i} \mathbf{j}} \theta_{\mathbf{k} \mathbf{l}}=-4 \delta_{(\mathbf{k}}^{\mathbf{i}} \delta_{\mathbf{l})}^{\mathbf{j}}, \quad D^{\mathbf{i} \mathbf{j}} \theta_{\mathbf{i j}}=-12, \\
& D^{\alpha \beta} \theta_{\alpha \beta}=-12, \quad D_{\alpha \beta} \theta_{\gamma \delta}=-4 \varepsilon_{(\alpha \gamma} \varepsilon_{\beta) \delta} \text {, } \\
& D^{3 \alpha \mathbf{i}} \theta^{4}=-36 \theta^{\alpha \mathbf{i}}, \quad D^{3 \alpha \mathbf{i}} \theta_{\beta \mathbf{j}}^{3}=9 \delta_{\beta}^{\alpha} \delta_{\mathbf{j}}^{\mathbf{i}}, \quad D^{4} \theta^{4}=144 \text {, } \\
& \bar{D}^{\mathbf{i j}} \bar{\theta}^{4}=-12 \bar{\theta}^{\mathbf{i j}}, \quad \bar{D}_{\mathbf{i j}} \bar{\theta}^{3 \dot{\alpha} \mathbf{k}}=6 \delta_{(\mathbf{i}}^{\mathbf{k}} \bar{\theta}_{\mathbf{j})}^{\dot{\alpha}}, \quad \bar{D}^{\mathbf{i j}} \bar{\theta}_{\dot{\alpha} \mathbf{j}}^{3}=-9 \bar{\theta}_{\dot{\alpha}}^{\mathbf{i}}, \\
& \bar{D}^{\mathbf{i j}} \bar{\theta}_{\mathbf{k} \mathbf{l}}=-4 \delta_{(\mathbf{k}}^{\mathbf{i}} \delta_{\mathbf{l})}^{\mathbf{j}}, \quad \bar{D}^{\mathbf{i j}} \bar{\theta}_{\mathbf{i j}}=-12, \\
& \bar{D}^{3 \dot{\alpha} \mathrm{i}} \bar{\theta}^{4}=-36 \bar{\theta}^{\dot{\alpha} \mathbf{i}}, \quad \bar{D}^{3 \dot{\alpha} \mathbf{i}} \bar{\theta}_{\beta \mathbf{j}}^{3}=-9 \delta_{\dot{\beta}}^{\dot{\alpha}} \delta_{\mathbf{j}}^{\mathbf{i}}, \quad \bar{D}^{4} \bar{\theta}^{4}=144, \\
& D^{4}\left(\theta^{4} X\right)|=144 X|, \quad \bar{D}^{4}\left(\bar{\theta}^{4} \bar{X}\right)|=144 \bar{X}|, \\
& D^{4}\left(\theta_{\alpha \mathbf{i}}^{3} X^{\alpha \mathbf{i}}\right)\left|=36 D_{\alpha \mathbf{i}} X^{\alpha \mathbf{i}}\right|, \quad \bar{D}^{4}\left(\bar{\theta}_{\dot{\alpha} \mathbf{i}}^{3} \bar{X}^{\dot{\alpha} \mathbf{i}}\right)\left|=-36 \bar{D}_{\dot{\alpha} \mathbf{i}} \bar{X}^{\dot{\alpha} \mathbf{i}}\right|, \\
& D^{4}\left(\theta_{\mathbf{i j}} X^{\mathbf{i j}}\right)\left|=-12 D_{\mathbf{i j}} X^{\mathbf{i j}}\right|, \quad \bar{D}^{4}\left(\bar{\theta}_{\mathbf{i j}} \bar{X}^{\mathbf{i j}}\right)\left|=-12 \bar{D}_{\mathbf{i j}} \bar{X}^{\mathbf{i j}}\right|, \\
& D^{4}\left(\theta_{\alpha \beta} X^{\alpha \beta}\right)\left|=-12 D_{\alpha \beta} X^{\alpha \beta}\right|, \quad \bar{D}^{4}\left(\bar{\theta}_{\dot{\alpha} \dot{\beta}} \bar{X}^{\dot{\alpha} \dot{\beta}}\right)\left|=-12 \bar{D}_{\dot{\alpha} \dot{\beta}} \bar{X}^{\dot{\alpha} \dot{\beta}}\right|, \\
& D^{4}\left(\theta_{\alpha \mathbf{i}} X^{\alpha \mathbf{i}}\right)\left|=4 D_{\alpha \mathbf{i}}^{3} X^{\alpha \mathbf{i}}\right|, \quad \bar{D}^{4}\left(\bar{\theta}_{\dot{\alpha} \mathbf{i}} \bar{X}^{\dot{\alpha} \mathbf{i}}\right)\left|=4 \bar{D}_{\dot{\alpha} \mathbf{i}}^{3} \bar{X}^{\dot{\alpha} \mathbf{i}}\right| .
\end{aligned}
$$




\section{References}

[1] J. Wess and B. Zumino, Nucl. Phys. B70 (1974) 139.

[2] S. Ferrara and B. Zumino, Nucl. Phys. B87 (1975) 174.

[3] M.T. Grisaru, in Cargese Lectures, 1979, eds. M.Levy, D.Deser (Plenum, 1979), p. 130.

[4] V. Novikov, M. Shifman, A. Vainstein and V. Zakharaov, Nucl. Phys. B229 (1983) 381; Phys. Lett. B166 (1986) 329.

[5] M.T. Grisaru and P. West, Nucl. Phys. B254 (1985) 249.

[6] M. Sohnius and P. West, Phys. Lett. B100 (1981) 45.

[7] P. Howe, K.S. Stelle and P.C. West, Phys. Lett. B124 (1983) 55.

[8] P.C. West, in Proceedings of the 1983 Shelter Island II Conference on Quantum Field Theory and the Fundamental Problems of Physics; edited by R. Jackiw, N. Khuri, S. Weinberg and E. Witten (M.I.T. Press).

[9] N. Seiberg and E. Witten, Nucl. Phys. B426 (1994) 19 [Erratum B430 (1994) 485] [hep-th/9407087.

[10] R. Flume, M. Magro, L. O'Raifeartaigh, O. Schnetz and I. Sachs, Nucl. Phys. B494 (1997) 331 hep-th/9611123.

[11] M. Magro, L. O'Raifeartaigh and I. Sachs, Nucl. Phys. B508 (1997) 433 hepth/9704027.

[12] V. Ogievetsky and E. Sokatchev, Nucl. Phys. B127 (1977) 309.

[13] A. Salam and J. Strathdee, Phys. Rev. D11 (1975) 1521; Nucl. Phys. B86 (1975) 142.

[14] V. Ogievetsky and E. Sokatchev, Sov. J. Nucl. Phys. 28(3) (1978).

[15] P. Howe, K.S. Stelle and P.K. Townsend, Nucl. Phys. B192 (1981) 332.

[16] K. Shizuya, Phys. Rev. D35 (1987) 1848.

[17] H. Osborn, Annals Phys. 272 (1999) 243 hep-th/9808041.

[18] I.L. Buchbinder and S.M. Kuzenko, Ideas and Methods of Supersymmetry and Supergravity, IOP Publ., Bristol and Philadelphia, 1995, Revised Edition 1998.

[19] T.E. Clark, O. Piguet and K. Sibold, Nucl. Phys. B143 (1978) 445; O. Piguet and K. Sibold, Renormalized Supersymmetry. The Perturbation Theory Of N=1 Supersymmetric Theories In Flat Space-Time, Boston, Usa: Birkhaeuser ( 1986) 346 p. ( Progress In Physics, 12).

[20] P.S. Howe and P.C. West, Nucl. Phys. B486 (1997) 425 hep-th/9607239. 
[21] S. Ferrara and P. van Nieuwenhuizen, Phys. Lett. B74 (1978) 333.

[22] V. Akulov, D. Volkov and V. Soroka, Theor. Math. Phys. 31 (1977) 12;

M.F. Sohnious and P. West, Phys. Lett. B105 (1981) 353.

[23] P. Breitenlohner, Nucl. Phys. B124 (1977) 500.

[24] S. J. Gates, M.T. Grisaru and W. Siegel, Nucl. Phys. B203 (1982) 189.

[25] A. Galperin, V. Ogievetsky and E. Sokatchev, Nucl. Phys. B252 (1985) 435.

[26] B. de Wit, J.W. van Holten and A. Van Proyen, Nucl. Phys. B167 (1980) 186; Nucl. Phys. B148 (1981) 77;

B. de Wit, R. Philippe and A. Van Proyen, Nucl. Phys. B219 (1983) 143.

[27] B. de Wit and A. Van Proyen, Nucl. Phys. B245 (1984) 186;

B. de Wit, P.G. Lauwers and A. Van Proyen, Nucl. Phys. B255 (1985) 569.

[28] L. Castellani, P. van Niewenhuizen and S.J. Gates, Phys. Rev. D22 (1980) 2364;

S.J. Gates, Nucl. Phys. B176 (1980) 397; Phys. Lett. B96 (1980) 305.

[29] P. Howe, Nucl. Phys. B199 (1982) 309.

[30] M. Müller, Consistent Classical Supergravity Theories, LNP 336 (Springer, Berlin, 1989).

[31] J. Wess and J. Bagger, Supersymmetry and Supergravity, Second edition, Princeton Series in Physics.

[32] M. Sohnius, Proc. 2nd Tutzing Symp. (1976) vol. 2, ed. L.Castell, M. Drieschner and C. von Weizsäcker, (Carl Hanser Verlag, Munich).

[33] W. Lang, Nucl. Phys B179 (1981) 106.

[34] B.P. Conlong and P.C. West (1993) unpublished; P.C. West, hep-th/9805055.

[35] P.S. Howe and G.G. Hartwell, Class. Quant. Grav. 12 (1995) 1823.

[36] J.H. Park, Nucl. Phys. B 559 (1999) 455 [hep-th/9903230.

[37] K.S. Stelle and P.C. West, Phys. Lett B74 (1978) 330.

[38] M.T. Grisaru, in Proceedings of Supersymmetry and Supergravity, Trieste 1984, p. 90 .

[39] W. Siegel, Phys. Lett. B85 (1979) 333.

[40] B. de Wit and M. Rocek, Phys. Lett. B109 (1982) 439.

[41] B. Chibisov and M. Shifman, Phys. Rev. D 56 (1997) 7990 [Erratum-ibid. D 58 (1997) 109901] [hep-th/9706141].

[42] G.W. Gibbons and P.K. Townsend, Phys. Rev. Lett. 83 (1999) 1727 hepth/9905196. 
[43] L. Brink, J.H. Schwarz and J. Scherk, Nucl. Phys. B121 (1977) 77;

R. Grimm, M. Sohnius and J. Wess, Nucl. Phys. B133 (1978) 275.

[44] E. Witten and D. Olive, Phys. Lett. B78 (1978) 97.

[45] S. Wolf, Mod. Phys. Lett. A14 (1999) 2789 [hep/th-9905194].

[46] A. Iorio, Phys. Lett. B487 (2000) 171 hep-th/9905069.

[47] M. F. Sohnius, Phys. Lett. B81 (1979) 8.

[48] A.W. Fisher, Nucl. Phys. B229 (1983) 142.

[49] H.D. Dahmen, S. Marculescu and L. Szymanowski, Nucl. Phys. B383 (1992) 110.

[50] S.J. Gates and W. Siegel, Nucl. Phys. B195 (1982) 39.

[51] B. de Wit, R. Philippe and A. Van Proeyen, Nucl. Phys. B219 (1983) 143.

[52] K.S. Stelle, in Proceedings of the Nuffield Workshop, Quantum Structure of Space and Time, p. 337, August 1981, edited by M.J. Duff and C.J. Isham.

[53] S. Kuzenko and S. Theisen, Class. Quant. Grav. 17 (2000) 665-696 hepth/9907107.

[54] A. Iorio, L. O'Raifeartaigh, I. Sachs and C. Wiesendanger, Nucl. Phys. B495 (1997) 433 [hep-th/9607110].

[55] A.S. Galperin, E.Ivanov, S.Kalitzin, V. Ogievetsky and E. Sokatchev, Class. Quant. Grav. 1 (1984) 469. 